\title{
Properties of a dehydroalanine analog of glutathione: A reactive electrophilic busulfan metabolite
}

\author{
Cody J. Peer \\ West Virginia University
}

Follow this and additional works at: https://researchrepository.wvu.edu/etd

\section{Recommended Citation}

Peer, Cody J., "Properties of a dehydroalanine analog of glutathione: A reactive electrophilic busulfan metabolite" (2009). Graduate Theses, Dissertations, and Problem Reports. 2940.

https://researchrepository.wvu.edu/etd/2940

This Dissertation is protected by copyright and/or related rights. It has been brought to you by the The Research Repository @ WVU with permission from the rights-holder(s). You are free to use this Dissertation in any way that is permitted by the copyright and related rights legislation that applies to your use. For other uses you must obtain permission from the rights-holder(s) directly, unless additional rights are indicated by a Creative Commons license in the record and/ or on the work itself. This Dissertation has been accepted for inclusion in WVU Graduate Theses, Dissertations, and Problem Reports collection by an authorized administrator of The Research Repository @ WVU.

For more information, please contact researchrepository@mail.wvu.edu. 


\title{
Properties of a Dehydroalanine Analog of Glutathione: A Reactive Electrophilic Busulfan Metabolite
}

\author{
Cody J. Peer
}

\author{
Dissertation \\ Submitted to the School of Pharmacy \\ at West Virginia University \\ In partial fulfillment of the requirements \\ For the degree of \\ Doctor of Philosophy \\ In Pharmaceutical and Pharmacological Sciences
}

Patrick S. Callery, Ph.D., Chair

William P. Petros, Pharm.D., FCCP.

Robert K. Griffith, Ph.D.

Peter M. Gannett, Ph.D.

Suzanne C. Bell, Ph.D.

Department of Basic Pharmaceutical Sciences

Morgantown, WV

2009

Keywords: Dehydroalanine; Glutathione; Glutathione-S Transferase; Busulfan;

Reactive oxygen species.

Copyright 2009 Cody J. Peer 


\begin{abstract}
Properties of a Dehydroalanine Analog of Glutathione: A Reactive Electrophilic Busulfan Metabolite
\end{abstract}

Cody J. Peer

Gamma-Glutamyldehydroalanylglycine (EdAG) is a dethiolated, electrophilic metabolite of glutathione (GSH) derived from the Phase II conjugation of GSH with busulfan catalyzed by glutathione S-transferase (GST). It was demonstrated in this dissertation that EdAG could be successfully synthesized and shown to be a metabolite of busulfan from in vitro metabolism by enzymes in human liver cytosol. The electrophilic EdAG was reactive toward the cellular thiols cysteine and glutathione (GSH), and toward cysteine residues in reduced bovine insulin B-chain and bovine serum albumin in vitro, as well. EdAG was demonstrated to be a reversible noncompetitive inhibitor of GSTs, specifically GSTA1-1 at micromolar concentrations up to 1 $\mathrm{mM}$. EdAG at high concentration $(10 \mathrm{mM})$ was found to be an irreversible inhibitor of human GSTA1-1, although an EdAG-modified peptide was not observed. Inhibition of GST by a busulfan metabolite has the potential of altering busulfan pharmacokinetics or GSH cellular function. Since conversion of GSH to EdAG represents a loss of thiol-related redox properties and the gain of a captodative radical scavenging dehydroalanine group, the reactivity of EdAG with hydroxyl radical was evaluated. EdAG was shown to scavenge hydroxyl radical generated in the Fenton reaction in a concentration-dependent manner. The results indicate a stabilized carbon-based captodative radical intermediate in the reaction of EdAG with hydroxyl radical. In support of a captodative mechanism was the identification of a dimerized $\gamma$-glutamylserylglycine as a product in the reaction. Conversion of GSH from a redox active nucleophile to an electrophilic dehydroalanine is a significant change in chemical reactivity that may have biological implications for GSH and free radical biochemistry as well as busulfan therapeutics. 


\section{DEDICATION}

To my parents Larry and Maurine Peer and especially to the love of my life for their unconditional love and support 


\section{ACKNOWLEDGMENTS}

Completion of this dissertation is owed to a great many factors over my term at WVU. Faculty, fellow graduate students, staff, family, and friends have combined to offer advice, keep my sanity, and provide encouragement to press on when things didn't go smoothly.

My advisor, Dr. Patrick S. Callery, has been and continues to be a tremendous mentor for me. His vast knowledge and creativity have helped train me to strive to always learn and to never rest on laurels. His confidence in me is much appreciated and motivation to work hard so to not disappoint.

I am indebted to Dr. William P. Petros for the research opportunities he provided me in his lab. His willingness to teach me pharmacokinetic analysis and faith in me to complete the research independently are greatly appreciated.

Special thanks go to the following faculty: My Dissertation Committee members, William Petros, Robert Griffith, Peter Gannett, and Suzanne Bell, for critiquing my research and providing suggestions and advice to make me a better scientist; Dr. Stephen Leonard from NIOSH for providing access to his ESR instrument for free radical scavenging analysis as well as his advice and encouragement; Dr. Justin Hettick from NIOSH for providing access to his state-of-the-art instruments for proteomic analysis, and especially for his guidance and assistance with MALDI mass spectrometry.

Personal thanks go to my fellow graduate students in BPS and the department secretaries for providing comic relief and a comfortable work environment. I am thankful for the friends I've made at WVU that has made graduate school truly enjoyable. Lastly, but always first in my life, special thanks to Christy for her unconditional love and support. 


\section{TABLE OF CONTENTS}

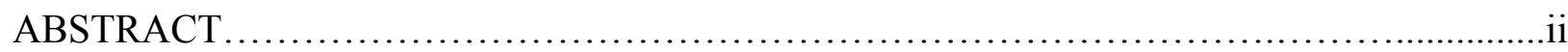

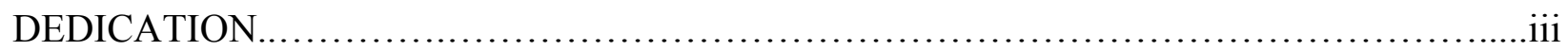

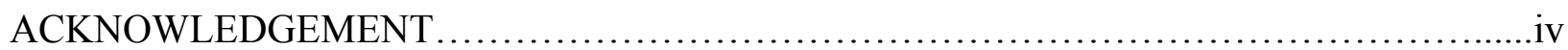

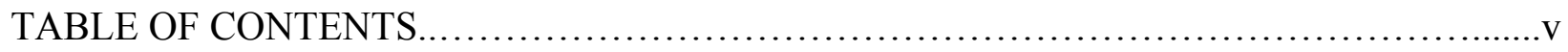

LIST OF FIGURES...............................................................

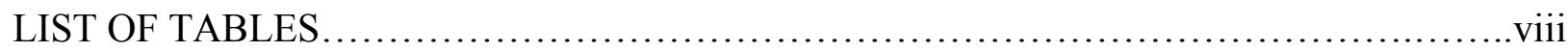

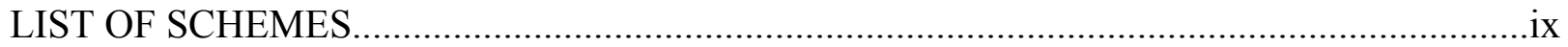

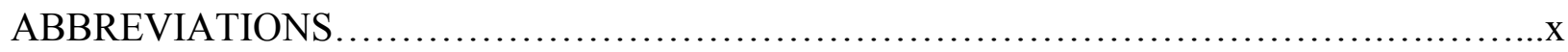

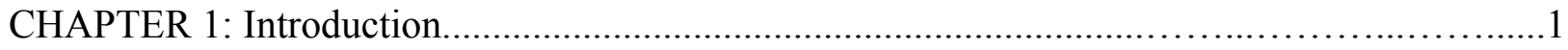

CHAPTER 2: Identification of EdAG as a metabolite from in vitro Busulfan-GSH conjugation

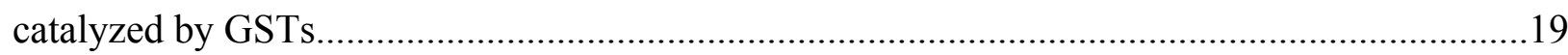

CHAPTER 3: Conjugation addition reactions of the dehydroalanine analog of GSH towards

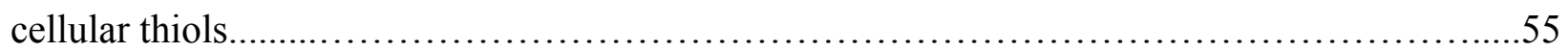

CHAPTER 4: Glutathione S-Transferase Inhibition by EdAG................................91

CHAPTER 5: Reactivity of EdAG as a Radical Scavenger.................................118

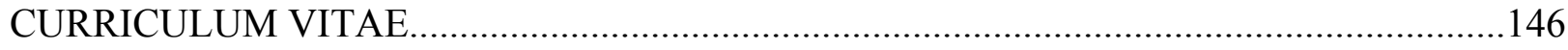




\section{LIST OF FIGURES}

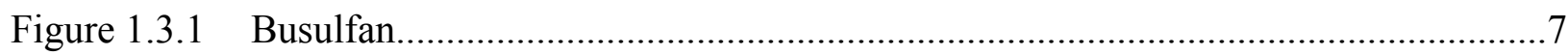

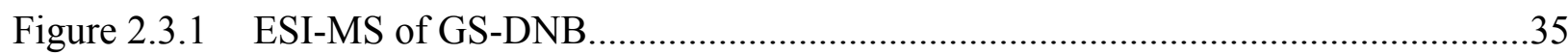

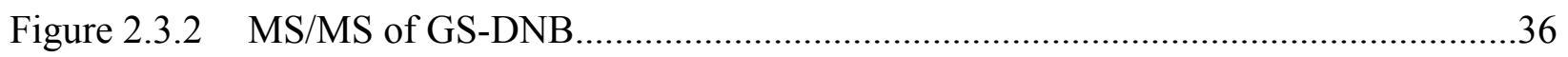

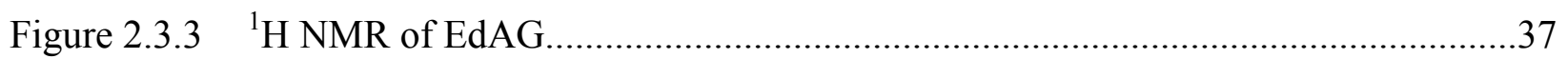

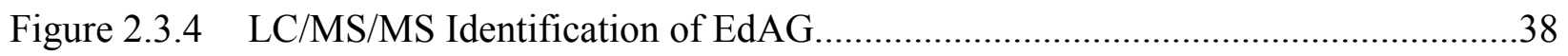

Figure 2.3.5 LC/MS of GSNO 24 hour degradation............................................................

Figure 2.3.6 Busulfan Incubation with Human Liver Cytosol after 2 hours incubation.............41

Figure 2.3.7 Busulfan Incubation with Human Liver Cytosol after 24 hours incubation...........43

Figure 3.3.1 MS/MS Fragmentation Pattern of S-( $\beta$-alanyl)-Glutathione..................................65

Figure 3.3.2 MS/MS Fragmentation Pattern of GSG.............................................................66

Figure 3.3.3 Nonenzymatic GSG formation after 5 minutes..................................................67

Figure 3.3.4 Nonenzymatic GSG formation after 180 minutes...............................................68

Figure 3.3.5 GSG formation in presence of GSTs after 180 minutes.......................................69

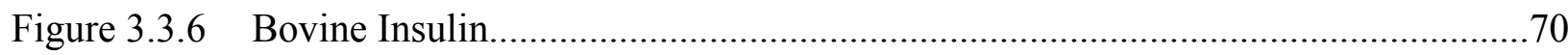

Figure 3.3.7 ESI mass spectrum of intact bovine insulin......................................................

Figure 3.3.8 ESI mass spectrum of bovine insulin B-chain.....................................................72

Figure 3.3.9 MALDI mass spectrum of bovine insulin B-chain..............................................73

Figure 3.3.10 ESI mass spectrum of the B-chain-2NEM adduct................................................74

Figure 3.3.11 MALDI mass spectrum of the B-chain-2NEM adduct...........................................75

Figure 3.3.12 MALDI mass spectrum of the B-chain-2EdAG adduct........................................76

Figure 3.3.13 MALDI mass spectra of mass range $m / z$ 1240-1300...........................................78 
Figure 3.3.14 MALDI mass spectra of mass range $m / z$ 1585-1675 ...................................80

Figure 3.3.15 MALDI mass spectra of mass range $m / z$ 1880-1960_.....................................81

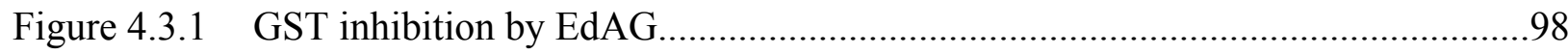

Figure 4.3.2 GSTA1-1 inhibition by EdAG................................................................. 100

Figure 4.3.3 Lineweaver-Burke plot suggests noncompetitive inhibition of

GSTA1-1 by EdAG.

Figure 4.3.4 Hanes-Woolf plot suggests noncompetitive inhibition of

GSTA1-1 by EdAG. 102

Figure 4.3.5 EdAG not inhibiting at substrate binding site............................................103

Figure 4.3.6 Irreversible inhibition of GSTA1-1 by EdAG and ethacrynic acid...................105

Figure 4.3.7 GSTA1-1 contains two cysteine residues in the solvent cleft...........................106

Figure 5.1.1 Glutathione redox mechanism for radicals..............................................119

Figure 5.1.2 Captodative mechanism of radical scavenging by dehydroalanines.................120

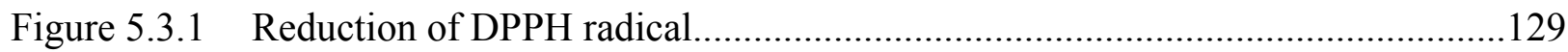

Figure 5.3.2 EPR Spectrum of Fenton Reaction with EdAG........................................130

Figure 5.3.3 EdAG-dependent scavenging by EPR ................................................131

Figure 5.3.4 Mechanism of hydroxyl radical trapping.................................................133

Figure 5.3.5 EPR Spectra of Fenton Reaction and POBN with EdAG.................................134

Figure 5.3.6 Accurate MS/MS spectrum of ESG dimer.................................................136

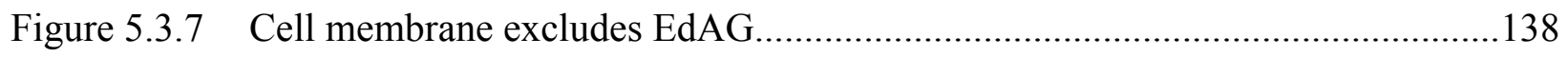




\section{LIST OF TABLES}

Table 3.1 BSA peptides from Trypsin Digestion...................................................... 77

Table 4.1 Enzyme Kinetic Parameters for GSTA1-1 Inhibition by EdAG Support Mixed

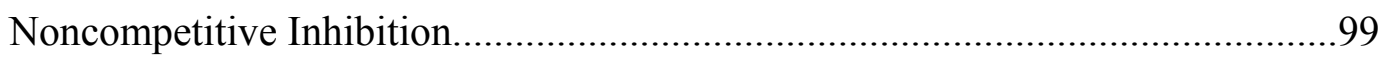

viii 


\section{LIST OF SCHEMES}

Scheme 1.1 Proposed Formation of EdAG from Phase II Metabolism of Busulfan..................8

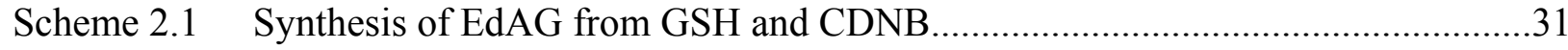

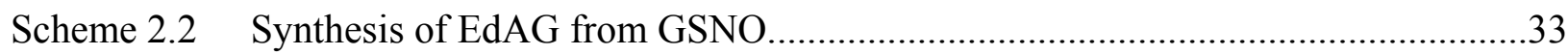

Scheme 5.3.1 Proposed Mechanism of Hydroxyl Radical Scavenging by EdAG...................132 


\section{ABBREVIATIONS}

Glutathione: GSH

1-Chloro-2,4-dinitrobenzene: CDNB

S-(2,4-Dinitrophenyl)-glutathione: GS-DNB

Gamma-glutamyldehydroalanylglycine: EdAG

Electrospray ionization mass spectrometry: ESI-MS

Radio frequency: RF

Direct current: DC

Quadrupole ion trap: QIT

Collision-induced dissociation: CID

Tandem mass spectrometry: MS/MS

Multi-stage mass spectrometry: $\mathrm{MS}^{\mathrm{n}}$

High performance liquid chromatography: HPLC

Proton nuclear magnetic resonance spectroscopy: ${ }^{1} \mathrm{H}$ NMR

Deuterium oxide: $\mathrm{D}_{2} \mathrm{O}$

Liquid chromatography/mass spectrometry: LC/MS

S-Nitrosylated glutathione: GSNO

Molecular ion: $\mathrm{MH}^{+}$

Glutathione S-transferase: GST

Dehydroalanines: Dha

Dehydrobutyrines: Dhb

Post-translational modification: PTM

Human serum albumin: HSA

Matrix-assisted laser desorption ionization-time of flight: MALDI-TOF

Glutathione-EdAG conjugate: GSG

Trizma buffer: Tris

Tris-(2-carboxyethyl)-phosphine: TCEP

N-Ethyl maleimide: NEM

Molecular weight: MW

Oxidized glutathione: GSSG

Drug absorption, distribution, metabolism, and elimination: ADME

N,N-dimethylacetamide: DMA

Tetrahydrothiophene: THT

Hepatic veno-occlusive disease: HVOD

Sinusoidal endothelial cells: SEC

Dimethylsulfoxide: DMSO

Acetonitrile: ACN

Human liver cytosol: HLC

Glutathione S-transferase isoform A1-1: GSTA1-1

Ultraviolet: UV

Ethacrynic acid: EA

Electron spin resonance: ESR 


\author{
Hydroxyl radical: ${ }^{\bullet} \mathrm{OH}$ \\ Superoxide radical: $\mathrm{O}_{2}^{-\bullet}$ \\ 5,5-dimethyl-1-pyrroline- $N$-oxide: DMPO \\ 1,1-diphenyl-2-picrylhydrazyl: DPPH \\ $\mathrm{N}$-acetyl cysteine: NAC \\ Phosphate-buffered saline: PBS \\ Serum free media: SFM \\ Dulbecco's modified eagle's medium: DMEM
}




\section{Chapter 1}

\section{Introduction}




\subsection{Drug Metabolism}

Nearly all complex organisms have built-in mechanisms for eliminating foreign compounds, or xenobiotics. Typically, the objective is to make those xenobiotics more water soluble in order to be eliminated. Involved in these processes are a variety of enzymes, substrates, and co-factors. The structure and properties of the xenobiotic determines the metabolic pathway that will metabolize the compound, however any single xenobiotic isn't necessarily limited to one specific pathway. There are two major routes, or phases, of metabolism that is dependent on the xenobiotic involved. Each phase employs certain enzymes, cofactors, and substrates to make the xenobiotic more polar and thus eliminated.

Phase I metabolism usually occurs in the liver or gut lumen, where xenobiotics are subjected to oxidation, reduction, or hydrolysis by enzymes such as the cytochrome P450 (CYP450) superfamily of enzymes and flavin monooxygenases (FMOs). There are a variety of metabolic mechanisms used to make the xenobiotic more polar and prepare it for elimination or further metabolism. The phase I-mediated mechanisms include, but are not limited to, side chain oxidation, aromatic ring oxidation, methyl oxidation, heterocyclic ring oxidation, N-dealkylation, O-dealkylation, S-dealkylation, deamination, N-oxidation, sulfoxidation, and azoreduction.

Phase II metabolism has the same basic function as phase I, that being to make the xenobiotic more polar to eliminate it from the organism. Phase II metabolism can occur on either a previously unmetabolized xenobiotic or one that underwent phase I metabolism. Phase II metabolic processes include, but are not limited to, glucuronidation, sulfation, acetylation, and conjugation with the abundant endogenous cellular nucleophilic tripeptide, glutathione (GSH). 


\subsubsection{Phase II Conjugation with Glutathione}

Phase II conjugation of xenobiotics with GSH is the result of catalysis by the glutathione S-transferase (GST) superfamily of enzymes. The addition of glutathione to endogenous or xenobiotic hydrophobic electrophiles render those adducts more water-soluble and thus, more readily eliminated from the body. Glutathione adducts are also subject to cellular efflux and further metabolism by the Mercapturic acid pathway, leading to polar cysteine-S-conjugates. There are many different sub-families of the GSTs based on their subcellular localization as either membrane-bound, mitochondrial, or cytosolic. Nomenclature of the different classes of GSTs is based on Greek letters (e.g. alpha, pi, mu), but abbreviated with Roman capital letters (e.g. A, P, M) (1). The membrane-bound classes of GSTs include three isoforms: microsomal I, microsomal II, and leukotriene $\mathrm{C}_{4}\left(\mathrm{LTC}_{4}\right)$, that are structurally very different from their cytosolic counterparts (2). Not much is known about these three classes of GSH transferases, however they are nonetheless important in detoxification of certain electrophilic compounds. The cytosolic classes of GSTs have been studied much more extensively and thus more structural and mechanistic information is known. The classes of the soluble (cytosolic) GST isoforms that are known include, but may not be limited to if new classes have since been discovered, alpha, zeta, theta, mu, pi, sigma, and omega (3).

The genetic origin has been extensively studied to determine the similarities between the classes of the GST superfamily of enzymes. GST isoforms of the same class (e.g. alpha) are classified together because their genes are located in very close proximity to each other on the same chromosome (3). GSTs are also dimeric proteins, but can only dimerize with isoforms within the same class. For example, the human GST alpha class (hGSTA) contains two major isoforms, hGSTA1 and hGSTA2. Between these two isoforms, three different dimeric proteins 
can form; the homodimeric hGSTA1-1 and hGSTA2-2, or the heterodimeric hGSTA1-2. The genes for each isoform is written italicized, e.g. hGSTA1.

Most of the seven soluble classes of GSTs have been crystallized and their quaternary and secondary subunit structures revealed. Cytosolic GSTs are all slightly different in terms of their primary amino acid sequence and tissue abundance, but all have similar protein folding and overall quaternary structure. The C-terminal domain accounts for two thirds of the protein structure and consists mostly of alpha helices (2). One of the major structural differences among the soluble GST classes is the substrate specificity for the electrophilic substrate to be conjugated by GSH. The hydrophobic substrate binding site, or H-site, is structurally unique for each isoenzyme within each class. The H-site is located within the crevice that forms between the Nterminal domain I and the C-terminal domain II. The N-terminal domain consists of mixtures of $\alpha$-helices and $\beta$-sheets and account for the remaining one third of the overall protein structure. Cytosolic GSTs all serve the same purpose of conjugating GSH to electrophilic substrates, thus all soluble GST classes need to bind GSH. Domain I contains similar secondary structural features across cytosolic GST classes because this accounts for the GSH binding site, or G-site. There are some differences in domain I between the classes in terms of the GSH recognition residues among classes and species, namely different residues responsible for docking and activating the GSH in the G-site. Most GST classes utilize a tyrosine residue positioned near the docked GSH to form hydrogen bonds to the thiol to deprotonate and stabilize the thiolate. Only theta class GSTs utilize a serine residue to hydrogen bond GSH (4). In human GSTA1-1, Arg15 and Tyr9 both form hydrogen bonds to the thiol of GSH when docked in the G-site (5). This helps lower the pKa of GST-bound GSH from 9 to about 6.6 (6). 


\subsection{Glutathione}

Glutathione (GSH) is an abundant cellular tripeptide consisting of L-glutamic acid, Lcysteine, and glycine. It is present in millimolar concentrations intracellularly and in micromolar concentrations in plasma and urine. It is the major source of cysteine and thiols in animals and plants. It is synthesized intracellularly, first by the rate-limiting addition of L-glutamate and Lcysteine by the ATP-dependent $\gamma$-glutamylcysteine synthetase $(7,8)$, followed by addition of glycine to the C-terminal end of $\gamma$-glutamylcysteine by the ATP-dependent glutathione synthetase (9). The cysteine residue contains a reactive and nucleophilic thiol side chain (-SH). The major roles of glutathione, as they pertain to this dissertation research, are two-fold: as a cellular nucleophile to conjugate and detoxify electrophilic compounds (in reactions catalyzed by GSTs), and as a cellular redox agent that scavenges reactive oxygen species (ROS) (10). Other roles of GSH include autoimmunity and transhydrogenation with compounds such as ascorbic acid (11). Glutathionylation of proteins occurs during states of oxidative stress and is categorized as a redox mechanism involving GSH. Often times, glutathionylated hemoglobin is a biomarker for oxidative stress (12).

Glutathione plays a major role in maintaining redox equilibrium in a cell. During normal physiological conditions for yeast cytoplasm, there are low steady state levels of ROS, thus oxidative stress is low (13). Most glutathione exists in the reduced form (GSH at pH 7.4, pKa 9), the levels of which are about 3000-fold more than the oxidized glutathione (GSSG) levels due to efficient reduction mechanisms (14). Normal cellular functions of mitochondria, peroxisomes, and other physiological cellular oxidative process require the need for redox mechanisms for their constant removal. There are many glutathione-dependent enzymes and mechanisms responsible for reduction of cytosolic ROS species, including GSH-dependent thiol peroxidases, 
thioredoxin, and glutaredoxin (15). Thioredoxin and glutaredoxin are primarily responsible for maintaining protein thiol oxidation in the cytosol. Another enzyme, glutathione reductase (GR), plays a major role in cellular redox state by reducing the oxidized GSSG back to GSH for recycling and continued redox scavenging. GR is a homodimeric enzyme containing FAD that requires NADPH for electron donation and is a very efficient reaction, with GSSG having a relatively low binding affinity $\left(K_{M}=55 \mu \mathrm{M}\right)$ considering the millimolar glutathione concentrations found intracellularly (16).

Phase II conjugation of xenobiotics with GSH is catalyzed by the GST superfamily of enzymes. Among those xenobiotics that undergo this metabolism are numerous anticancer agents, specifically DNA alkylating agents, because they are electrophilic. Compounds must be electrophilic in order to be conjugated by nucleophilic atoms on DNA nucleotides (e.g. N7 of guanine bases). There are five classifications of DNA alkylating groups based on their structure and anticancer mechanism: nitrogen mustards, nitrosoureas, ethylenimines, triazenes, and alkyl sulfonates (17). Of interest to this dissertation research was the alkyl sulfonate anticancer agent busulfan, due to its phase II GSH conjugation and resulting metabolite that is a dethiolated glutathione.

\subsection{Busulfan}

Busulfan is a bifunctional anticancer agent that is classified as an alkyl sulfonate as part of the larger group of anticancer drugs known as DNA-alkylating agents. Busulfan contains sulfonate groups on both ends of the molecule (Figure 1.3.1) that allow it to be reactive towards DNA on both ends. The carbons adjacent to the electron-withdrawing sulfonate groups are electrophilic and are susceptible to nucleophilic attack. 


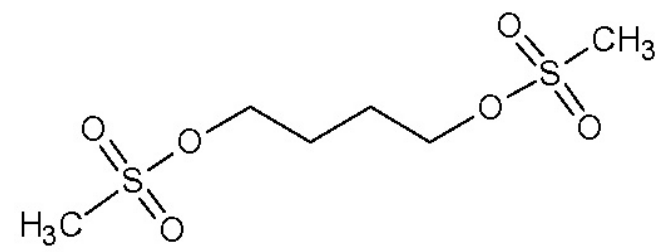

Figure 1.3.1 Busulfan

This allows either end of busulfan to be conjugated by nucleophilic atoms on DNA nucleotides, such as the N7 atom on guanine bases. Busulfan was developed in the 1950s and first used clinically as a chemotherapeutic in 1959. Initially, busulfan was used to treat chronic myelogenous leukemia $(18,19)$ but is also now used in high doses along with cyclophosphamide for myeloablative conditioning therapy prior to bone marrow transplants and hematopoietic stem cell transplants (20), as well as polycythemia rubra vera (21) and essential thrombocythemia (22).

Busulfan is metabolized by phase II conjugation with GSH catalyzed by the GST superfamily of enzymes, but predominantly cytosolic GSTA1-1 in liver hepatocytes (23). The hydrophobic busulfan, which is typically administered orally or through intravenous emulsion solutions, is a good substrate for the hydrophobic binding site (H-site) on GSTA1-1. The product formed by GSTs, which is released back to the intrahepatocellular cytosol, is an unstable sulfonium ion ( $\gamma$-glutamyl- $\beta$-[S-tetrahydrothiophenium]alanylglycine; GS $\left.{ }^{+} \mathrm{THT}\right)$ (24). It was shown by Younis et al that $\mathrm{GS}^{+}$THT slowly degrades at $\mathrm{pH} 7.4$ by a base-catalyzed $\beta$ elimination reaction of tetrahydrothiophene (THT), and the degradation occurs faster at $\mathrm{pH} 8.4$ (25). The portion of $\mathrm{GS}^{+}$THT remaining after elimination of THT is a dethiolated analog of GSH, $\gamma$-glutamyldehydroalanylglycine (EdAG) as depicted in Scheme 1.1. 

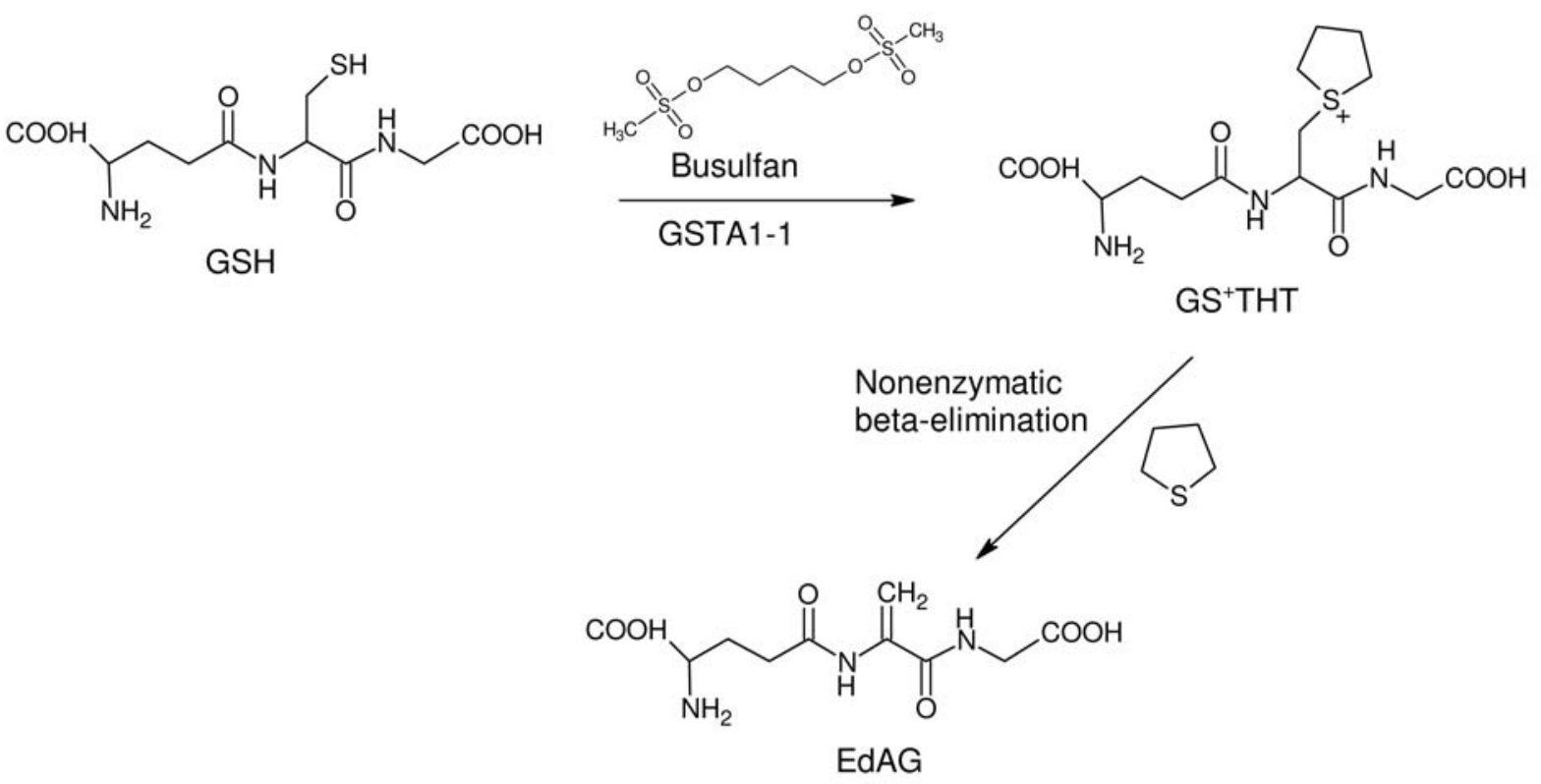

Scheme 1.1 Busulfan Metabolism Catalyzed by GSTA1-1

EdAG contains a dehydroalanyl residue in lieu of the cysteinyl residue in GSH that potentially alters the chemical properties of the molecule. The moiety of glutathione that enabled the molecule to have such important and wide-ranging functions, the nucleophilic thiol side chain on the cysteinyl residue, is now replaced with an electrophilic vinylic carbon. Dehydroalanines are not a novel species, however much has already been studied including formation and occurrences in vivo.

\subsection{Dehydroalanines}

\subsubsection{Formation of Dehydroalanines}

Dehydroalanines are amino acid residues found within proteins or peptides. They can be formed either naturally by cellular processes (e.g. post-translational modifications) or chemically (e.g. food processing). Dehydroalanines (Dha) and dehydrobutyrines (Dhb) are mainly formed 
from serine and threonine residues, respectively, by the same mechanism under conditions of high $\mathrm{pH}$ and temperature (26). Dha residues formed from cysteine residues in vivo are rare aside from chemically-induced non-physiological reactions (27).

One such setting that provides these conditions is during food processing, where many proteins in meat and other processed foods contain cysteine residues that undergo a basecatalyzed $\beta$-elimination induced by heat and/or the alkaline environment (28). Another more naturally-occurring scenario where the dehydration of serine and threonine can occur is during an in situ alkaline environment, where basic amino acids near a serine or threonine could induce a $\beta$-elimination forming Dha and Dhb, respectively (29). Posttranslationally-modified peptides made by ribosomes within Gram-positive bacteria contain many Dha or Dhb residues that can be further modified by nucleophilic addition by a cysteine residue to form a nonreducible lanthionine thioether (Ala-S-Ala). Proteins that contain these lanthionines produced by ribosomes in Gram-positive bacteria have antibiotic properties and are referred to as lantibiotics (30).

\subsubsection{Lantibiotics are peptides that contain dehydroalanine crosslinks with cysteines}

The 1928 discovery of a substance produced by a colony of bacteria that inhibited its further growth was the birth of lantibiotics (31). Gram positive bacteria produce a variety of peptides called bacteriocins, of which those that contain lanthionine or $\beta$-methyl lanthionine crosslinks are called lantibiotics. When lantibiotics are ribosomally synthesized and posttranslationally modified, numerous serines and threonines are transformed into dehydroalanine or dehydrobutyrine residues, respectively. These serine/threonine residues are phosphorylated by serine/threonine kinases followed by dehydration by dehydratases and 
cyclization with nearby cysteine residues (forming the lanthionine) by cyclases before proteolysis of the leader peptide (32). Serines and threonines within the leader portion of the peptide are not subject to modification to Dha and Dhb, respectively, however within the propeptide portion, serines are twice as likely to avoid modifications as threonines (33). The incidence of modification and cyclization are dependent upon the amino acids flanking the modified dehydrated amino acids. It was determined that hydrophobic, nonaromatic amino acids more often flank serines and threonines that become modified (33). Dha and Dhb can then react with cysteine residues flanked by less hydrophobic amino acid residues near the C-terminus within the same peptide to form the lanthionine or $\beta$-methyl lanthionine crosslinks, respectively, that are integral in the efficacy of the lantibiotic as an antimicrobial agent. The thioether crosslinks that form the bridges are very important to the structural stability and antimicrobial efficacy of the lantibiotic $(34,35)$. The structural rigidity of lantibiotics is dependent upon the thioether rings, as well as the peptide's resistance to proteolysis and heat, which are also ringdependent (36). The LanB dehydratases are nonspecific concerning stereochemistry, but the LanC cyclases, in addition to the stereospecificity of the peptide sequence, play a role in determining ring formation (37-39). Due to their stability and resistance to proteolysis, lantibiotics have not shown as much bacterial resistance as conventional antibiotics have. Lantibiotics could serve as a novel antibiotic for drug-resistant strands of bacteria.

Lantiobiotics are classified into two types, depending on their shape and functionality. Type A lantibiotics are typically amphiphilic and elongated peptides and target the cell membrane of bacteria to form pores that induces bacterial death (40). Type B are globular proteins that bind to lipids in bacterial cell membranes that effectively halt production of membrane lipids by inhibiting the enzymes that produce them. Lantibiotics are cationic peptides 
that are effective in much lower concentrations than those cationic peptides typically manufactured by mammals, insects, fish, and amphibians (41). Type A lantibiotics use LanB dehydratases and LanC cyclases to transform serine/threonine residues to Dha/Dhb, respectively, and type B lantibiotics use LanM proteins that execute the same reactions in type B lantibiotics as LanB and LanC enzymes for type A lantibiotics $(42,43)$. Based on the modes of action, which is determined by the type of lantibiotic (A or B), there are many lantibiotics that have potential in treating bacterial diseases like acne and MRSA (Nisin A, Lacticin 3147, Gallidermin, Epidermin, Mutacin 1140, Mersacidin, Actagardine) as well as inflammation (Duramycin) and regulation of blood pressure (Cinnamycin, Ancovenin) (44).

Nisin, the common food preservative, is the most known and studied lantibiotic. Due to its antibiotic properties and structure that enable it to avoid proteolysis and thermal degradation, it is widely used to preserve such foods as vegetables, canned goods, dairy products, and natural cheeses (45). Nisin contains many dehydroalanines that are not within thioether rings, thus they are susceptible to conjugation by nucleophiles outside of the peptide (e.g. glutathione). It is not as potent in preserving meat because it is suspected that the high GSH content in meat tissue can form other thioethers with dehydro amino acids in the peptide (46). Synthetic dehydroalanines occur in proteins of food during food processing due to the high temperature and alkali conditions.

\subsection{Research Objectives}

Busulfan is known to be metabolized via GSTA1-1 catalyzed phase II glutathione conjugation to form the unstable sulfonium $\mathrm{GS}^{+} \mathrm{THT}$. The degradation product of the enzyme metabolized product, GS ${ }^{+}$THT, is the dehydroalanine analog of glutathione, EdAG (Scheme 1.1). 
EdAG has been previously shown to bind GSTs (25). The electrophilic nature of the dehydroalanine renders EdAG reactive towards cellular nucleophiles. Also, it was suggested that like other dehydroalanine-containing compounds, EdAG could also react with radicals, such as hydroxyl radical. Thus, it was the focus of the research presented here to determine the following:

1. Identify EdAG as an in vitro product of busulfan metabolism with GSTs based on LC/MS characteristics compared to a synthesized EdAG standard.

2. Determine the reactivity of EdAG toward the cellular nucleophiles GSH and Lcysteine, as well as cysteine residues in proteins using insulin and bovine serum albumin as model proteins.

3. Determine if EdAG is inhibiting GSTs and elucidate the mechanism of EdAG-induced inhibition of GSTs over a wide concentration range.

4. Characterize the radical scavenging activity of EdAG towards the ROS hydroxyl radical using electron paramagnetic resonance (EPR) spectroscopy and mass spectrometry. 


\section{REFERENCES}

(1) Mannervik, B., Awasthi, Y. C., Board, P. G., Hayes, J. D., Di, I. C., Ketterer, B., Listowsky, I., Morgenstern, R., Muramatsu, M., Pearson, W. R., and . (1992) Nomenclature for human glutathione transferases. Biochem. J. 282 ( Pt 1), 305306.

(2) Armstrong, R. N. (1997) Structure, catalytic mechanism, and evolution of the glutathione transferases. Chem. Res. Toxicol. 10(1), 2-18.

(3) Mannervik, B., Board, P. G., Hayes, J. D., Listowsky, I., and Pearson, W. R. (2005) Nomenclature for mammalian soluble glutathione transferases. Methods Enzymol. $401,1-8$.

(4) Board, P. G., Coggan, M., Wilce, M. C., and Parker, M. W. (1995) Evidence for an essential serine residue in the active site of the Theta class glutathione transferases. Biochem. J. 311 ( Pt 1), 247-250.

(5) Bjornestedt, R., Stenberg, G., Widersten, M., Board, P. G., Sinning, I., Jones, T. A., and Mannervik, B. (1995) Functional significance of arginine 15 in the active site of human class alpha glutathione transferase A1-1. J. Mol. Biol. 247(4), 765-773.

(6) Graminski, G. F., Kubo, Y., and Armstrong, R. N. (1989) Spectroscopic and kinetic evidence for the thiolate anion of glutathione at the active site of glutathione Stransferase. Biochemistry 28(8), 3562-3568. 
(7) Griffith, O. W., and Mulcahy, R. T. (1999) The enzymes of glutathione synthesis: gamma-glutamylcysteine synthetase. Adv. Enzymol. Relat Areas Mol. Biol. 73, 209-67, xii.

(8) Snoke, J. E., Yanari, S., and Bloch, K. (1953) Synthesis of glutathione from gammaglutamylcysteine. J. Biol. Chem. 201(2), 573-586.

(9) Snoke, J. E., and Bloch, K. (1955) Studies on the mechanism of action of glutathione synthetase. J. Biol. Chem. 213(2), 825-835.

(10) Hayes, J. D., and McLellan, L. I. (1999) Glutathione and glutathione-dependent enzymes represent a co-ordinately regulated defence against oxidative stress. Free Radic. Res. 31(4), 273-300.

(11) Perricone, C., De, C. C., and Perricone, R. (2009) Glutathione: a key player in autoimmunity. Autoimmun. Rev. 8(8), 697-701.

(12) Bursell, S. E., and King, G. L. (2000) The potential use of glutathionyl hemoglobin as a clinical marker of oxidative stress. Clin. Chem. 46(2), 145-146.

(13) Heeren, G., Jarolim, S., Laun, P., Rinnerthaler, M., Stolze, K., Perrone, G. G., Kohlwein, S. D., Nohl, H., Dawes, I. W., and Breitenbach, M. (2004) The role of respiration, reactive oxygen species and oxidative stress in mother cell-specific ageing of yeast strains defective in the RAS signalling pathway. FEMS Yeast Res. 5(2), 157-167.

(14) Ostergaard, H., Tachibana, C., and Winther, J. R. (2004) Monitoring disulfide bond formation in the eukaryotic cytosol. J. Cell Biol. 166(3), 337-345.

(15) Lopez-Mirabal, H. R., and Winther, J. R. (2008) Redox characteristics of the eukaryotic cytosol. Biochim. Biophys. Acta 1783(4), 629-640. 
(16) Massey, V., and Williams, C. H., Jr. (1965) On the reaction mechanism of yeast glutathione reductase. J. Biol. Chem. 240(11), 4470-4480.

(17) Dirven, H. A., van, O. B., and van Bladeren, P. J. (1996) Glutathione conjugation of alkylating cytostatic drugs with a nitrogen mustard group and the role of glutathione S-transferases. Chem. Res. Toxicol. 9(2), 351-360.

(18) Silver, R. T. (2003) Chronic myeloid leukemia. Hematol. Oncol. Clin. North Am. 17(5), 1159-1vii.

(19) Stone, R. M. (2004) Optimizing treatment of chronic myeloid leukemia: a rational approach. Oncologist. 9(3), 259-270.

(20) Russell, S., and Vowels, M. (1992) Busulphan, cyclophosphamide, and melphalan as conditioning therapy in allogeneic bone marrow transplants for acute lymphoblastic leukemia. Transplant. Proc. 24(1), 183.

(21) Mital, A., Czyz, J., and Hellmann, A. (1996) [Evaluation of treatment results in patients with polycythemia rubra vera]. Pol. Arch. Med. Wewn. 95(5), 453-458.

(22) Van de Pette, J. E., Prochazka, A. V., Pearson, T. C., Singh, A. K., Dickson, E. R., and Wetherley-Mein, G. (1986) Primary thrombocythaemia treated with busulphan. Br. J. Haematol. 62(2), 229-237.

(23) Czerwinski, M., Gibbs, J. P., and Slattery, J. T. (1996) Busulfan conjugation by glutathione S-transferases alpha, mu, and pi. Drug Metab Dispos. 24(9), 10151019.

(24) Roberts, J. J., and Warwick, G. P. (1961) The mode of action of alkylating agents. II. Studies of the metabolism of myleran. The reaction of myleran with some naturally occurring thiols in vitro. Biochem. Pharmacol. 6, 205-216. 
(25) Younis, I. R., Elliott, M., Peer, C. J., Cooper, A. J., Pinto, J. T., Konat, G. W., Kraszpulski, M., Petros, W. P., and Callery, P. S. (2008) Dehydroalanine analog of glutathione: an electrophilic busulfan metabolite that binds to human glutathione S-transferase A1-1. J. Pharmacol. Exp. Ther. 327(3), 770-776.

(26) Finley, J. W., and Friedman, M. (1977) New amino acid derivatives formed by alkaline treatment of proteins. Adv. Exp. Med. Biol. 86B, 123-130.

(27) Bernardes, G. J., Chalker, J. M., Errey, J. C., and Davis, B. G. (2008) Facile conversion of cysteine and alkyl cysteines to dehydroalanine on protein surfaces: versatile and switchable access to functionalized proteins. J. Am. Chem. Soc. 130(15), $5052-5053$.

(28) Friedman, M. (1999) Chemistry, biochemistry, nutrition, and microbiology of lysinoalanine, lanthionine, and histidinoalanine in food and other proteins. $J$. Agric. Food Chem. 47(4), 1295-1319.

(29) Nakanishi, T., Sato, T., Sakoda, S., Yoshioka, M., and Shimizu, A. (2004) Modification of cysteine residue in transthyretin and a synthetic peptide: analyses by electrospray ionization mass spectrometry. Biochim. Biophys. Acta 1698(1), 4553.

(30) Chatterjee, C., Paul, M., Xie, L., and van der Donk, W. A. (2005) Biosynthesis and mode of action of lantibiotics. Chem. Rev. 105(2), 633-684.

(31) Rogers, L. A., and Whittier, E. O. (1928) Limiting Factors in the Lactic Fermentattion. J. Bacteriol. 16(4), 211-229. 
(32) Okeley, N. M., Paul, M., Stasser, J. P., Blackburn, N., and van der Donk, W. A. (2003) $\mathrm{SpaC}$ and $\mathrm{NisC}$, the cyclases involved in subtilin and nisin biosynthesis, are zinc proteins. Biochemistry 42(46), 13613-13624.

(33) Rink, R., Kuipers, A., de, B. E., Leenhouts, K. J., Driessen, A. J., Moll, G. N., and Kuipers, O. P. (2005) Lantibiotic structures as guidelines for the design of peptides that can be modified by lantibiotic enzymes. Biochemistry 44(24), 88738882.

(34) Bierbaum, G., Szekat, C., Josten, M., Heidrich, C., Kempter, C., Jung, G., and Sahl, H. G. (1996) Engineering of a novel thioether bridge and role of modified residues in the lantibiotic Pep5. Appl. Environ. Microbiol. 62(2), 385-392.

(35) van, K. C., Breukink, E., Rollema, H. S., Bongers, R. S., Kosters, H. A., de, K. B., and Kuipers, O. P. (2000) Engineering a disulfide bond and free thiols in the lantibiotic nisin Z. Eur. J. Biochem. 267(3), 901-909.

(36) Kuipers, O. P., Bierbaum, G., Ottenwalder, B., Dodd, H. M., Horn, N., Metzger, J., Kupke, T., Gnau, V., Bongers, R., van den, B. P., Kosters, H., Rollema, H. S., de Vos, W. M., Siezen, R. J., Jung, G., Gotz, F., Sahl, H. G., and Gasson, M. J. (1996) Protein engineering of lantibiotics. Antonie Van Leeuwenhoek 69(2), 161169.

(37) Burrage, S., Raynham, T., Williams, G., Essex, J. W., Allen, C., Cardno, M., Swali, V., and Bradley, M. (2000) Biomimetic synthesis of lantibiotics. Chemistry. 6(8), $1455-1466$.

(38) Zhou, H., and van der Donk, W. A. (2002) Biomimetic stereoselective formation of methyllanthionine. Org. Lett. 4(8), 1335-1338. 
(39) Zhu, Y., Gieselman, M. D., Zhou, H., Averin, O., and van der Donk, W. A. (2003) Biomimetic studies on the mechanism of stereoselective lanthionine formation. Org. Biomol. Chem. 1(19), 3304-3315.

(40) Jung, G., and Sahl, H. G. (1991) Nisin and Novel Lantibiotics. (Jung, G., and Sahl, H. G., Eds.) pp 1-34, Escom,

(41) Breukink, E., Wiedemann, I., van, K. C., Kuipers, O. P., Sahl, H., and de, K. B. (1999) Use of the cell wall precursor lipid II by a pore-forming peptide antibiotic. Science 286(5448), 2361-2364.

(42) Sahl, H. G., and Bierbaum, G. (1998) Lantibiotics: biosynthesis and biological activities of uniquely modified peptides from gram-positive bacteria. Annu. Rev. Microbiol. $52,41-79$.

(43) Siezen, R. J., Kuipers, O. P., and de Vos, W. M. (1996) Comparison of lantibiotic gene clusters and encoded proteins. Antonie Van Leeuwenhoek 69(2), 171-184.

(44) Ryan, M. P., Hill, C., and Ross, R. P. (2002) Peptide antibiotics - discovery, modes of action and applications. (Dutton, C. J., Haxell, M. A., McArthur, M. A. I., and Wax, R. G., Eds.) pp 193-242, Marcel Dekker,

(45) ves-Broughton, J., Blackburn, P., Evans, R. J., and Hugenholtz, J. (1996) Applications of the bacteriocin, nisin. Antonie Van Leeuwenhoek 69(2), 193-202.

(46) Rose, N. L., Sporns, P., Dodd, H. M., Gasson, M. J., Mellon, F. A., and McMullen, L. M. (2003) Involvement of dehydroalanine and dehydrobutyrine in the addition of glutathione to nisin. J. Agric. Food Chem. 51(10), 3174-3178. 


\section{Chapter 2}

Identification of EdAG as a metabolite from in

vitro Busulfan-GSH conjugation catalyzed by

GSTs 


\section{INTRODUCTION}

\subsubsection{Busulfan}

Busulfan, or 1,4-butanediol dimethanesulfonate, is a bifunctional DNA alkylating agent classified as an alkyl sulfonate. Busulfan, like other alkylating agents, induces its effects by donating an alkyl group to nucleophilic biomolecules, such as DNA. The mechanism of action of busulfan is to bind DNA in rapidly-dividing cells (e.g. cancer cells) to prevent replication. The primary site of action on DNA is the N7 atom on guanine nucleotides, although other atoms provide secondary sites of action. Busulfan forms intrastrand cross-links at the 5'-GA-3' sequence, as well as monoalkylation on the N7 of guanine, in a dose-dependent manner (1).

Busulfan was introduced in 1959 as a chemotherapeutic agent to treat myeloproliferative disorders. Initially, busulfan was used to treat chronic myelogenous leukemia $(2,3)$ but is also now used in high doses along with cyclophosphamide for myeloablative conditioning therapy for bone marrow transplants and hematopoietic stem cell transplants (4), as well as polycythemia rubra vera (5) and essential thrombocythemia (6).

\subsubsection{Busulfan Absorption, Distribution, Metabolism, and Elimination (ADME)}

Busulfan pharmacokinetics have been extensively studied as the oral formulation, and only more recently studied as the intravenous (i.v.) formulation. Busulfan disposition following oral dosing can best be described by a one-compartment open model. Oral formulations were originally marketed as Myleran ${ }^{\circledR}$ by GlaxoSmithKline with relatively good bioavailability (mean $80 \%$ ), but varied greatly. Recently, the organic solvent N,N-dimethylacetamide (DMA) has been used to solvate the highly lipophilic busulfan into aqueous solution for i.v. administration. The 
market name for the i.v. formulation is Busulfex ${ }^{\circledR}$ (Otsuka), and is composed of $6 \mathrm{mg} / \mathrm{kg}$ busulfan solvated in 33\% DMA (v/v) and 67\% polyethylene glycol $400(\mathrm{v} / \mathrm{v})$. Each Busulfex ${ }^{\circledR}$ vial is then diluted further to a busulfan concentration of $0.5 \mathrm{mg} / \mathrm{mL}$ in $0.9 \%$ saline or $5 \%$ dextrose with a $\mathrm{pH}$ range of 3.4-3.9. Comparisons of bioavailability and exposure between oral and i.v. formulations have shown that a $1 \mathrm{mg} / \mathrm{kg}$ oral dose is bioequivalent to a $0.8 \mathrm{mg} / \mathrm{kg}$ i.v. dose of busulfan (7). There has been a recent switch in busulfan formulation usage from oral to i.v. because the intravenous route is easier to perform therapeutic drug monitoring to ensure busulfan exposure remains below the target level of $1,500 \mu \mathrm{M} \bullet \mathrm{min}$ to avoid toxicity such as hepatic veno-occlusive disease (HVOD) (8).

Oral busulfan has very high interpatient pharmacokinetic variability that is affected by various conditions such as circadian rhythmicity, disease states, age, and dosage (9-12), with either a zero-order absorption (13) or first-order absorption $(14,15)$. Time to maximum absorption $\left(\mathrm{t}_{\max }\right)$ range between $0.5-1$ hour, however some patients can absorb busulfan immediately whereas others (up to $25 \%$ ) have lag times of up to an hour (16). Busulfan disposition is best described by a one-compartment open model. Roughly $1 \%$ of busulfan is excreted in urine as unchanged (13). Typical elimination half-lives did not vary significantly between children and adults, with average half-lives in children of 2.5 hours (17-19), and in adults of 2.75 hours (20).

Orally-administered busulfan is absorbed from the gastrointestinal (GI) tract into the hepatic portal circulation, where it undergoes first-pass metabolism from either the gut or liver, or both, prior to distribution into the systemic circulation. GSTA1-1, the major enzyme responsible for busulfan metabolism, is mainly located in the liver but is also found in the intestinal wall epithelia. The first-pass effect can ultimately lower the bioavailability of an oral 
drug, thus decreasing its exposure and altering its pharmacokinetics. In the systemic circulation, busulfan has a relatively low binding affinity to plasma proteins, where $32.4 \%$ of busulfan dose is irreversibly bound (21). Intravenous busulfan avoids first-pass metabolism and thus exposure and clearance are not affected by this effect. Busulfan is also evenly distributed between plasma and blood cells, where $46.9 \%$ of the busulfan dose was irreversibly bound to blood cells to induce a myeloablative effect (21). A significant portion (20\%) of busulfan is able to cross the blood brain barrier (22). Busulfan concentrations in the cerebrospinal fluid were significant enough to be monitored and elimination parameters calculated (23-25).

The major route of busulfan metabolism is glutathione $S$-transferase (GST)-catalyzed GSH conjugation, which forms a relatively unstable sulfonium ion ( $\gamma$-glutamyl- $\beta$ - $[S-$ tetrahydrothiophenium]alanylglycine; $\mathrm{GS}^{+} \mathrm{THT}$ ) (26). The major GST isoform responsible for busulfan conjugation to GSH is the cytosolic GSTA1-1 (27). The sulfonium is further metabolized by either of two pathways. The first pathway is the Mercapturic acid pathway (MCA), where the sulfonium is cleaved by a variety of enzymes that produce the final product of a $N$-acetylcysteine-S-sulfonium conjugate (28). The purpose of the MCA pathway is to detoxify toxic compounds, however the product of the second to last step of the MCA pathway is a cysteine $S$-conjugate. This compound can either be $N$-acetylated with Acetyl CoA by $N$ acetyltransferase, or can be a substrate for a class of enzymes called cysteine-S-conjugate $\beta$ lyases. These enzymes cleave the thioether linkage, forming a dehydrocystine (later rearranges to $\alpha$-imino acid before hydrolyzing to pyruvate and ammonium) and a sulfur-containing moiety, that is potentially toxic when reabsorbed from the gut lumen or renal proximal tubule recirculation. The second pathway that the GS ${ }^{+}$THT sulfonium can follow is through a nonenzymatic $\beta$-elimination of tetrahydrothiophene (THT) (26), which produces an electrophilic 
dehydroalanine analog of glutathione, $\gamma$-glutamyldehydroalanylglycine (EdAG) (29) (Scheme $1.1)$.

This dissertation will focus on the busulfan metabolite EdAG that is suggested to be reactive towards biological nucleophiles due the electrophilic dehydroalanine residue. The potential reactivity of EdAG could be implicated in a number of disease states where GSH depletion or macromolecular conjugation is associated, such as hepatic veno-occlusive disease.

\subsubsection{Hepatic Veno-Occlusive Disease Associated with Busulfan}

Hepatic veno-occlusive disease, or HVOD, is caused by the destruction of sinusoidal endothelial cells (SECs) resulting from myeloablative conditioning therapies of busulfan and cyclophosphamide during bone marrow and hematopoietic stem cell transplants. Prior to therapeutic drug monitoring, anywhere between $30-50 \%$ of patients receiving busulfan conditioning therapy developed HVOD, with a third of those having such a severe form that they do not survive $(16,30)$. HVOD is difficult to predict because oral administration of busulfan has such a high interpatient variability in absorption and exposure. In myeloablative therapy, busulfan is normally administered first, followed by a washout period of at least 24 hours, then treatment with cyclophosphamide. Busulfan has been shown to deplete intracellular hepatic GSH levels in murine models by as much as 50\% (31) and it is known that the major metabolite of the active form of the prodrug cyclophosphamide is detoxified by GSH, further depleting GSH. Because both drugs require GSH for detoxification along with an oxidative environment that could saturate GR-catalyzed GSSG reduction to GSH, it is logical that intracellular hepatic GSH could be reduced to levels that are insufficient to protect the SECs in the liver. 
Busulfan causes a depletion in GSH stores due to its metabolism and renders hepatocytes and SECs vulnerable to injury caused by the co-administered cyclophosphamide. It is uncertain whether HVOD is caused by toxicity related to circulating busulfan or from busulfan metabolites. However, Srivastava et al suggested busulfan metabolites as responsible for GSH depletion and increasing the exposure of cyclophosphamide as a possible explanation for the elevated risk of HVOD (32). Tetrahydrothiophene (THT) is the lipophilic metabolite of busulfan that undergoes further oxidation by Cytochrome P450s and has been shown to be toxic to SECs and hepatocytes, thus most research on busulfan-mediated HVOD has focused on THT. Clinical trials involving busulfan-cyclophosphamide co-administration have found a correlation between busulfan exposure levels and HVOD that would suggest a maximum busulfan exposure $(<1,500$ $\mu \mathrm{mol} \times \mathrm{min} / \mathrm{L}$ ) that greatly reduces chances of inducing HVOD (9). Therapeutic drug monitoring after the first dose of busulfan is useful in determining busulfan exposure and allows for dose adjustments to reduce risks of HVOD.

Therapeutic drug monitoring requires bioanalytical instrumentation to identify and quantify busulfan and its metabolites in biological fluids in order to determine safe and effective dosing and exposure limits. Typically, such instruments as high performance liquid chromatography (HPLC) coupled to electrospray ionization mass spectrometry (ESI-MS), or LC/MS, are employed. HPLC is used to separate analytes from salts and other impurities in the matrix, while MS can structurally identify and quantify the analytes.

\subsubsection{Electrospray Ionization Mass Spectrometry (ESI-MS)}

A common ionization technique used with mass spectrometry when interfaced with liquid chromatography is electrospray ionization (ESI), a soft ionization technique that ionizes analytes 
in the solution phase before nebulization to the gaseous phase without degrading or denaturing the analyte (33). ESI was first published by John Fenn in 1989 (34), but was the cumulative work of many. The principles behind ESI were laid down by Lord Rayleigh in 1882, and then fully realized into production by Malcolm Dole in 1968, who discovered that when there are solubilized ions in a droplet, as the solvent evaporates and the droplet becomes smaller, the charge density increases to a critical point where the droplet disperses into numerous smaller droplets. This phenomenon is known as the Rayleigh limit, where Coulombic repulsion was greater that surface tension and allowed the droplet to disperse into many tiny droplets (35). The Rayleigh limit then applies to each offspring droplet and the process is repeated as the ionized droplets are "sprayed" from the narrow-bore capillary needle.

Electrospray ionization can be applied to both positive and negative ions, however positive ESI is more common due to the ability of nitrogen atoms to add protons, with the majority of marketed pharmaceuticals possessing amide and amine functional groups, where the oxygen atom of the amide is protonated. In positive ESI, a positive charge, usually $5 \mathrm{kV}$, is applied to the cone and is usually called the cone or spray voltage. This serves to help repel the positively-charged analytes in droplets away from the cone and towards the mass analyzer. There also exists a counter electrode with either a small orifice or heated capillary, where a negative charge of significantly lower voltage is applied to attract the cationic analytes. An inert gas, usually nitrogen gas, is used as a sheath gas to desolvate the charged droplets on their way to the mass analyzer.

\subsubsection{ESI-MS with Ion Trap for structural analysis}


Ion trap (IT) mass analyzers are commonly used in conjunction with ESI to separate and analyze ions according to their mass to charge ratio $(\mathrm{m} / \mathrm{z})$. First invented in the $1950 \mathrm{~s}$, ITs are about the size of a softball and contain three electrodes, where an entrance and exit electrode surround a ring electrode. Together, these three electrodes apply a constant radio frequency (RF) signal with steadily increasing amplitude along with a direct current (DC) to the ring electrode. This forces the ions into an orbit that essentially "traps" the ions, with each ion traveling on its own orbit according to its $\mathrm{m} / \mathrm{z}$ ratio. Due to the kinetic energy associated with the ions, a damping gas (typically helium) is applied in the ion trap to cool the ions to a stable oscillating flight path or trajectory.

IT mass analyzers have the ability to eject all ions out of the mass analyzer except the ion of interest, which can then be subjected to collision-induced dissociation (CID) by bombardment with high energy helium atoms. Tandem mass spectrometry, or MS/MS, is frequently used to study the structure of molecules. IT mass analyzers also have the ability to perform multi-stage fragmentation $\left(\mathrm{MS}^{\mathrm{n}}\right)$, capable of fragmenting the same ion numerous cycles.

\subsubsection{Chapter Goals}

It has been logically assumed that EdAG is a degradation product of the major busulfan metabolite resulting from Phase II conjugation to GSH catalyzed by glutathione S-transferase, specifically isoform A1-1. When busulfan is metabolized by GSTA1-1, a sulfonium ion is

formed, $\gamma$-glutamyl- $\beta$-(S-tetrahydrothiophenium)alanylglycine, or $\mathrm{GS}^{+} \mathrm{THT}$, that is unstable at pH 7.4 and non-enzymatically degrades to THT and EdAG. While most research has focused on THT, there is a lack of literature on EdAG as a metabolite from busulfan. Thus, it was desired in this chapter to demonstrate that EdAG is a metabolite from GST-catalyzed busulfan conjugation 
with GSH using an in vitro model of human liver cytosol. First, a reference standard of EdAG will be synthesized to better identify the compound formed under physiological reaction conditions using LC/MS/MS. 


\section{EXPERIMENTAL PROCEDURES}

\subsubsection{Materials}

Glutathione, 1-chloro-2,4-dinitrobenzene (CDNB), secondary butanol, busulfan, Lcysteine, formic acid, DMSO, Tris buffer, $\mathrm{pH}$ 7.4, and Dowex-50W were purchased from SigmaAldrich (St. Louis, MO). Sodium bicarbonate, methanol (optima), acetonitrile (optima), isopropyl alcohol, and hydrochloric acid were purchased from Fisher Scientific (Pittsburgh, PA). All water used was deionized with a NANOpure ${ }^{\circledR}$ water purification system (Barnstead, San Jose, CA). Pooled human liver cytosol (HLC) from multiple donors, containing all cytosolic isoforms of GST (20 mg/mL total protein concentration) was purchased from BD Gentest (Bedford, MA). All water used was deionized with a NANOpure ${ }^{\circledR}$ water purification system (Barnstead, San Jose, CA).

\subsubsection{Instrumentation}

High-performance liquid chromatography (HPLC) was performed on a Shimadzu 10 Avp binary pump HPLC system equipped with a UV/Vis detector (Shimadzu Scientific Instruments, Columbia, MD). Mass spectrometry was performed using an electrospray ionization (ESI) ion trap (IT) LCQ DECA (ThermoFisher Scientific, Waltham, MA), capable of multi-stage mass spectrometry (MS, MS/MS, $\mathrm{MS}^{3}$ ). Structural information was obtained using nuclear magnetic resonance (NMR) on a Varian $300 \mathrm{MHz}$ broadband spectrometer (Varian, Palo Alto, CA).

\subsubsection{Synthesis of $\gamma$-Glutamyldehydroalanylglycine (EdAG)}

\subsubsection{Synthesis of the Precursor $S$-(2,4-dinitrophenyl)-Glutathione (GS-DNB)}


EdAG was synthesized by the elimination of S-(2,4-dinitrobenzene) from $S-(2,4-$ dinitrophenyl)glutathione (GS-DNB). Thus, GS-DNB was first synthesized by the condensation of reduced glutathione (GSH) to 1-chloro-2,4-dinitrobenzene (CDNB) under basic conditions based on a modified method from Patchornik (36). Equimolar solutions of GSH (3.07 g in 40 $\mathrm{mL}$ of $\left.1 \mathrm{~N} \mathrm{NaHCO}_{3} ; 10 \mathrm{mmol}\right)$ and $\mathrm{CDNB}(2.02 \mathrm{~g}$ in $20 \mathrm{~mL}$ methanol; $10 \mathrm{mmol})$ were prepared and CDNB was added dropwise to GSH over 30 minutes with stirring. The solution was stirred at room temperature for one hour and then filtered to eliminate precipitates. The product was precipitated from solution by addition of $1 \mathrm{M} \mathrm{HCl}$ and collected by vacuum filtration, dried overnight in a vacuum desiccator, and recrystallized from hot water. To ensure the correct product was obtained, the yellow crystals were characterized by ESI-MS ( $\mathrm{m} / \mathrm{z} 474), \mathrm{MS} / \mathrm{MS}$ ( $\mathrm{m} / \mathrm{z}$ 399, $\mathrm{MH}^{+}$-glycine; $\mathrm{m} / \mathrm{z} 345, \mathrm{MH}^{+}$-pyroglutamic acid), melting point analysis (m.p. $189-191{ }^{\circ} \mathrm{C}$ )

and ${ }^{1} \mathrm{H}$ NMR in $\mathrm{d}_{6}$-DMSO. The following ${ }^{1} \mathrm{H}$ NMR resonances were monitored: $\delta 1.84(\mathrm{~m}, 1 \mathrm{H})$, $1.95(\mathrm{~m}, 1 \mathrm{H}), 2.32(\mathrm{~m}, 2 \mathrm{H}), 3.31-3.38(\mathrm{~m}, 2 \mathrm{H}), 3.60-3.65(\mathrm{~m}, 1 \mathrm{H}), 3.73(\mathrm{~m}, 1 \mathrm{H}), 4.61(\mathrm{~m}, 1 \mathrm{H})$ $7.97(\mathrm{~d}, 1 \mathrm{H}), 8.46(\mathrm{~d}, 1 \mathrm{H}), 8.71(\mathrm{~d}, 1 \mathrm{H})$, and $8.87 \mathrm{ppm}(\mathrm{m}, 2 \mathrm{H})$.

\subsubsection{Synthesis of EdAG from GS-DNB}

S-(2,4-Dinitrophenyl)glutathione was used as a precursor to $\gamma$ glutamyldehydroalanylglycine (EdAG) based on a modified method from Carthew et al (37). Two grams of GS-DNB were dissolved in $100 \mathrm{~mL}$ of $0.5 \mathrm{M} \mathrm{NaOH}$ and stirred for 30 minutes at room temperature. To remove impurities, the solution was extracted with $40 \mathrm{~mL}$ butanol and repeated a total of 5 times. The combined aqueous layers were diluted to $300 \mathrm{~mL}$ and approximately 15 grams of activated Dowex ${ }^{\circledR}-50 \mathrm{~W}$ ion exchange resin (hydrogen form) was added to remove sodium ions. The Dowex ${ }^{\circledR}$ was activated by dissolving the resin in $1 \mathrm{M} \mathrm{HCl}$ 
and dried. The $\mathrm{pH}$ of the solution dropped from 13 to 3.0, indicating exchange of $\mathrm{H}^{+} / \mathrm{Na}^{+}$. The aqueous solution was evaporated using either roto-evaporation or speed vacuum evaporation to collect a light brown solid. The obtained product was characterized by LC/MS/MS by the observance of a single absorbance peak at $254 \mathrm{~nm}$ corresponding to parent ion $\mathrm{m} / \mathrm{z} 274$ that was fragmented (MS/MS) to obtain characteristic daughter ions ( $\mathrm{m} / \mathrm{z} 256, \mathrm{MH}^{+}$-water; $\mathrm{m} / \mathrm{z} \mathrm{199}, \mathrm{MH}^{+}$glycine; $m / z$ 145, $\mathrm{MH}^{+}$-pyroglutamic acid). ${ }^{1} \mathrm{H}$ NMR in $\mathrm{D}_{2} \mathrm{O}$ was also performed and the following peaks were monitored: $\delta 2.06(\mathrm{~m}, 2 \mathrm{H}), 2.48(\mathrm{~m}, 2 \mathrm{H}), 3.69(\mathrm{~m}, 1 \mathrm{H}), 3.93(\mathrm{~s}, 2 \mathrm{H})$, and $5.76(\mathrm{~d}, 2 \mathrm{H}) . \quad$ LC/MS/MS conditions performed were as follows: A Thermo Hybercarb column (100 x $2.1 \mathrm{~mm}$ I.D., $5 \mu \mathrm{m}$, ThermoFisher, Waltham, MA) was used to isolate the product from other constituents in the reaction mixture. A gradient of mobile phase A $(0.5 \%$ formic acid in 90/10, v/v, water/acetonitrile) and mobile phase B (0.1\% formic acid in 50/50, v/v, isopropanol/methanol) at $0.2 \mathrm{~mL} / \mathrm{min}$ was run from 0-5 minutes using $100 \% \mathrm{~A}$; from $5-7$ minutes, $0 \rightarrow 50 \% \mathrm{~B}$; from 7-9 minutes, $50 \rightarrow 0 \% \mathrm{~B}$; and from 9-15 minutes, $100 \% \mathrm{~A}$. An ESI ion trap mass spectrometer was operated in the positive ion MS/MS mode with a spray voltage of 5.2 $\mathrm{kV}$, capillary voltage of $30 \mathrm{~V}$, capillary temperature of $300^{\circ} \mathrm{C}$, a nitrogen sheath gas flow rate of 80 arbitrary units, and a selected reaction monitoring on $\mathrm{m} / \mathrm{z} 274( \pm 1.5 \mathrm{amu})$ with $30 \%$ collision energy. 
<smiles>NC(CCC(=O)NC(CS)C(=O)NCC(=O)O)C(=O)O</smiles>
GSH

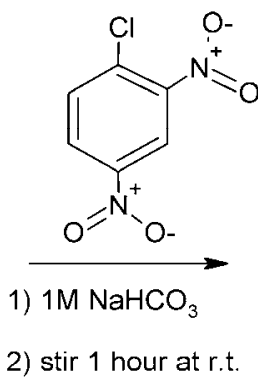

2) stir 1 hour at $r$.<smiles>O=[N+]([O-])c1ccc(S)c([N+](=O)[O-])c1</smiles><smiles>NC(CCC(=O)NC(CSc1ccc([N+](=O)[O-])cc1[N+](=O)[O-])C(=O)NCC(=O)O)C(=O)O</smiles>

GS-DNB

1) $0.5 \mathrm{M} \mathrm{NaOH}$

2) stir for $30 \mathrm{~min}$<smiles>C=C(NC(=O)CCC(N)C(=O)OCc1ccccc1)C(=O)OCC(=O)O</smiles>

Scheme 2.1 Synthesis of EdAG from GSH and CDNB

\subsubsection{Formation of EdAG from S-Nitrosylated GSH (GSNO)}

GSNO has the potential to degrade into EdAG by the elimination of S-NO (S-nitric oxide). This could serve as a more efficient precursor to EdAG than GS-DNB without the hydrophobic impurities. The stability of GSNO under various $\mathrm{pH}$ conditions was assessed with the hypothesis that at high $\mathrm{pH}$, the GSNO will lose S-NO and EdAG will remain. Controls of GSH, NEM (trap GSH for more stable signal in LC/MS), and EdAG were incubated for 24 hours in a $37^{\circ} \mathrm{C}$ water bath in $100 \mathrm{mM}$ Tris buffer, $\mathrm{pH}$ 8.4. Aliquots were taken every hour for the first six hours, then again at 24 hours to determine baseline changes in stability using LC/MS, with GS-NEM serving as an internal standard. Then, GSNO and NEM were incubated over 24 hours and samples analyzed the first 5 hours and then again at 24 hours. The decrease in GSNO signal 
relative to EdAG was monitored to determine extent of GSNO degradation to EdAG. The presence of GS-NEM indicates that GSNO degraded to GSH, which was trapped by NEM. If GSNO was degrading to EdAG, the ratio of GSNO/EdAG would decrease. LC/MS in the positive electrospray single ion monitoring mode was used to determine relative quantities of the ions $m / z$ 433, $\mathrm{m} / \mathrm{z}$ 337, and $\mathrm{m} / \mathrm{z}$ 274, corresponding to GS-NEM, GSNO, and EdAG, respectively. Chromatographic separation was achieved using a gradient of mobile phase A $(0.5 \%$ formic acid, $0.1 \%$ trifluoroacetic acid in water) and mobile phase B $(0.1 \%$ formic acid, $0.1 \%$ trifluoroacetic acid in 50/50, v/v, methanol/water) as follows: $0 \rightarrow 15 \mathrm{~min}, 0 \rightarrow 40 \% \mathrm{~B} ; 15 \rightarrow 17 \mathrm{~min}, 40 \rightarrow 100 \%$; and $17 \rightarrow 20 \mathrm{~min}, 100 \rightarrow 0 \%$ B. A Thermo Hybercarb column $(100 \times 2.1 \mathrm{~mm}$ I.D., $5 \mu \mathrm{m}$, ThermoFisher, Waltham, MA) was used to separate the constituents in the incubation with a flow rate of $200 \mu \mathrm{L} / \mathrm{min}$. The first mass spectral scan event employed two simultaneous single ion monitoring (SIM) scans of m/z 274 (for EdAG) and m/z 337 (for GSNO) and was performed from $0 \rightarrow 12$ min. The second scan event was a single SIM on $m / z 433$ (for GS-NEM) from $12 \rightarrow 20 \mathrm{~min}$. 


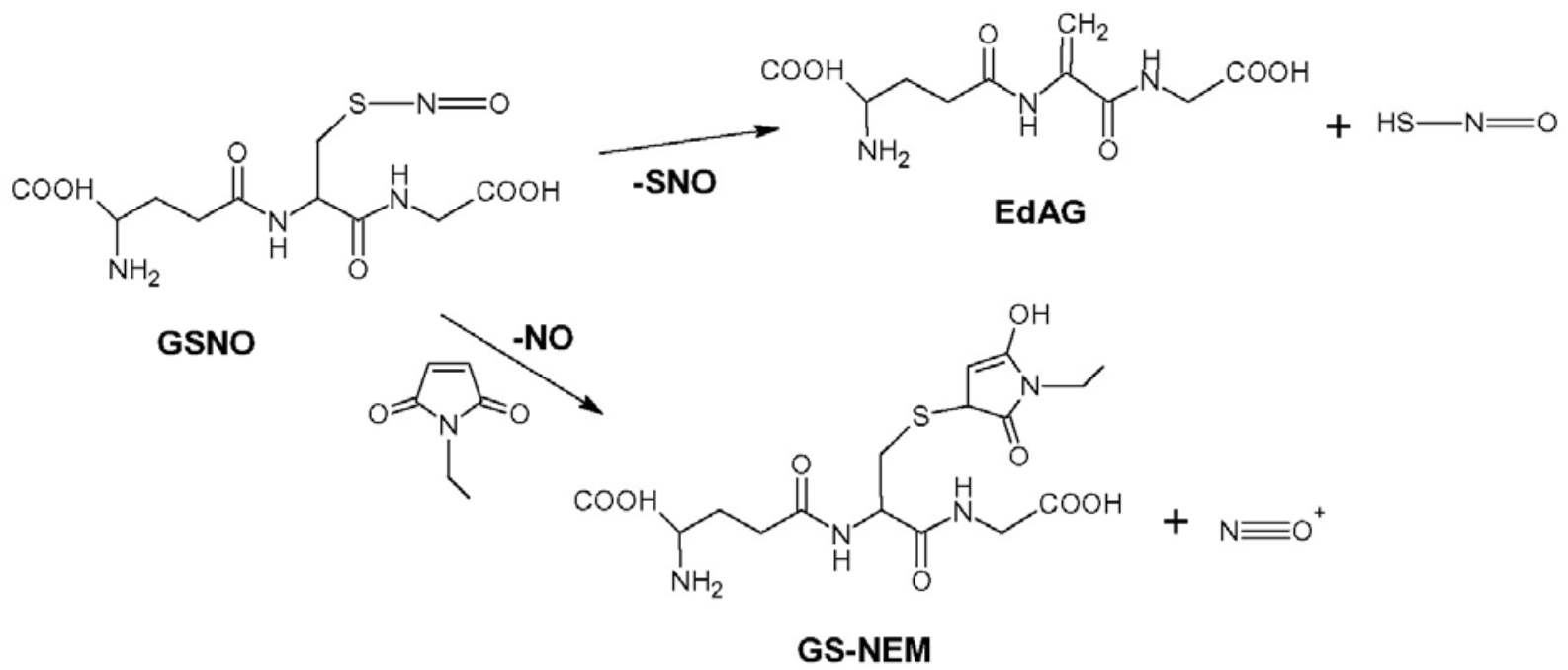

Scheme 2.2 Synthesis of EdAG from GSNO

\subsubsection{Identification of EdAG after in vitro busulfan metabolism}

Busulfan $(0-5 \mathrm{mM})$ was incubated with human liver cytosol (total protein $1.0 \mathrm{mg}$ ) in the presence of $1.0 \mathrm{mM}$ GSH in $100 \mathrm{mM}$ Tris buffer (pH 7.4, total volume $1.0 \mathrm{~mL}$ ). Busulfan was dissolved in DMSO and the total DMSO concentration was less than $1.0 \%(\mathrm{v} / \mathrm{v})$. The mixture containing human liver cytosol and GSH in buffer was preincubated for 5 minutes in a water bath maintained at $37^{\circ} \mathrm{C}$, and reactions started by the addition of busulfan. Changes in the levels of GSH, the enzymatic product GS ${ }^{+} \mathrm{THT}$, EdAG, and a nonreducible lanthionine thioether GSG (from GSH conjugation to EdAG) were monitored by liquid chromatography-mass spectrometry (LC/MS). Chromatographic separation of the polar compounds was achieved using a Thermo Hypercarb column (100 x 2.1 mm I.D., $5 \mu \mathrm{m}$ particle size) (ThermoFisher, Waltham, MA). A gradient of mobile phase A ( $0.5 \%$ formic acid in $90 / 10, \mathrm{v} / \mathrm{v}$, water/acetonitrile) and mobile phase $\mathrm{B}(0.1 \%$ formic acid in $50 / 50, \mathrm{v} / \mathrm{v}$, isopropanol $/$ methanol $)$ at $0.2 \mathrm{~mL} / \mathrm{min}$ was run from $0-5$ minutes using $100 \% \mathrm{~A}$; from $5-7$ minutes, $0 \rightarrow 50 \% \mathrm{~B}$; from 7-9 minutes, $50 \rightarrow 0 \% \mathrm{~B}$; and from 9- 
15 minutes, $100 \%$ A. The following ions were monitored: $\mathrm{m} / \mathrm{z} 308, \mathrm{~m} / \mathrm{z} 362, \mathrm{~m} / \mathrm{z} 274$, and $\mathrm{m} / \mathrm{z}$ 581, corresponding to reduced GSH, GS ${ }^{+} \mathrm{THT}, \mathrm{EdAG}$, and GSG respectively. The structure of EdAG was confirmed using LC/MS/MS in a separate run that fragmented $m / z 274$ to monitor product ions of $m / z 256,199$, and 145, consistent with neutral losses of water, glycine, and pyroglutamic acid, respectively, based on a comparison of the mass spectrum and retention time of the metabolite with that of the chemically synthesized standard. The structure of GSG was also confirmed in a separate LC/MS/MS.. 


\section{RESULTS}

\subsubsection{Synthesis of $\gamma$-Glutamyldehydroalanylglycine (EdAG)}

EdAG was successfully synthesized from GSH and CDNB according to the method of Asquith and Carthew (37) modified by Younis et al (29). Typical product yields of the precursor, S-(2,4-dinitrophenyl)glutathione (GS-DNB), ranged from 54-85\% after recrystallization. ESI-MS was performed to confirm product identity by monitoring the molecular ion $\mathrm{m} / \mathrm{z} 474$ (Figure 2.3.1). Tandem mass spectrometry (MS/MS) was also performed for structural identification and the product ions of $\mathrm{m} / \mathrm{z} 345$ and $\mathrm{m} / \mathrm{z} 399$ were formed, corresponding to the $\mathrm{MH}^{+}$GS-DNB molecular ion with neutral losses of pyroglutamic acid and glycine, respectively (Figure 2.3.2).

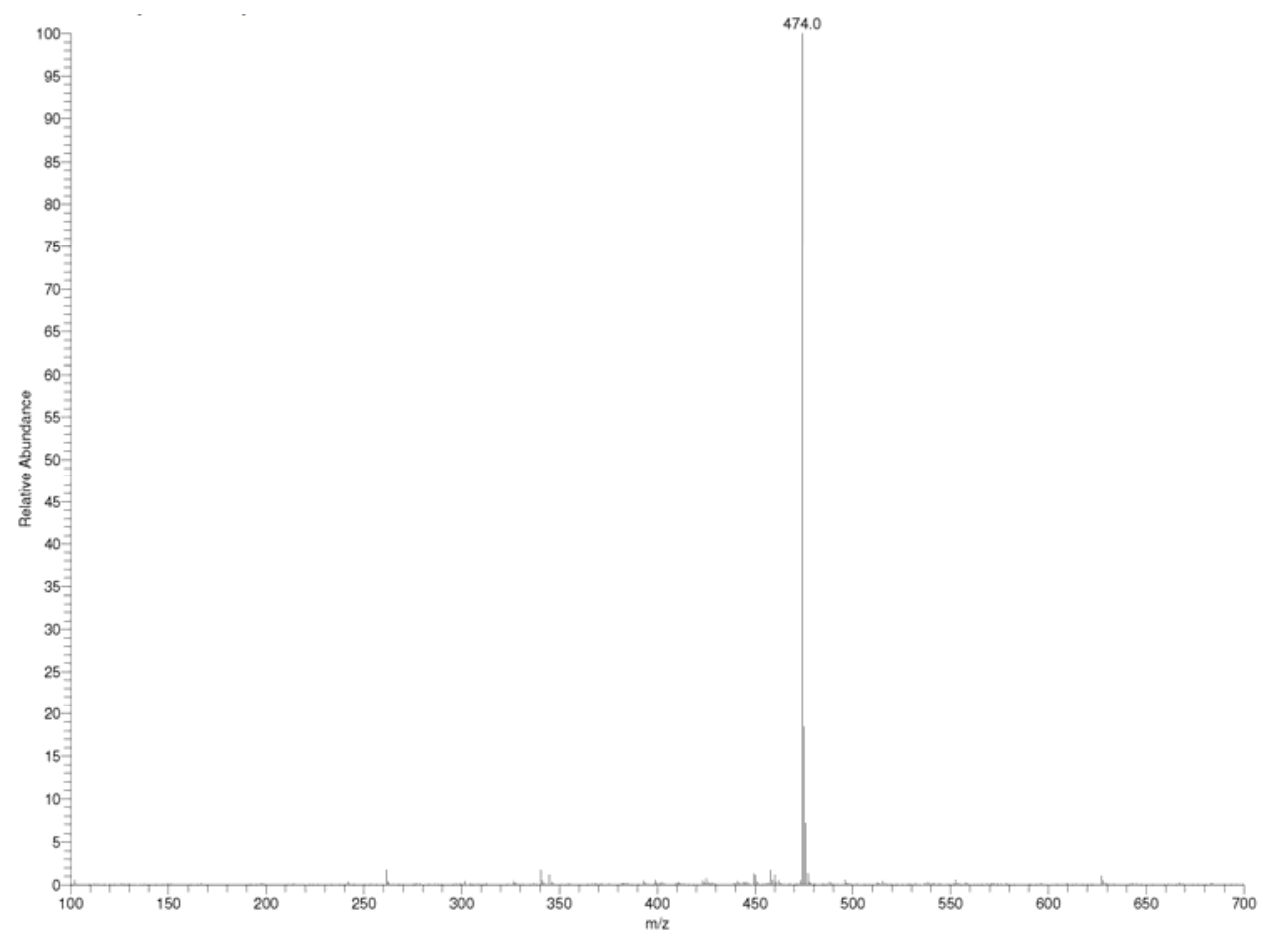

Figure 2.3.1 ESI-MS of GS-DNB 


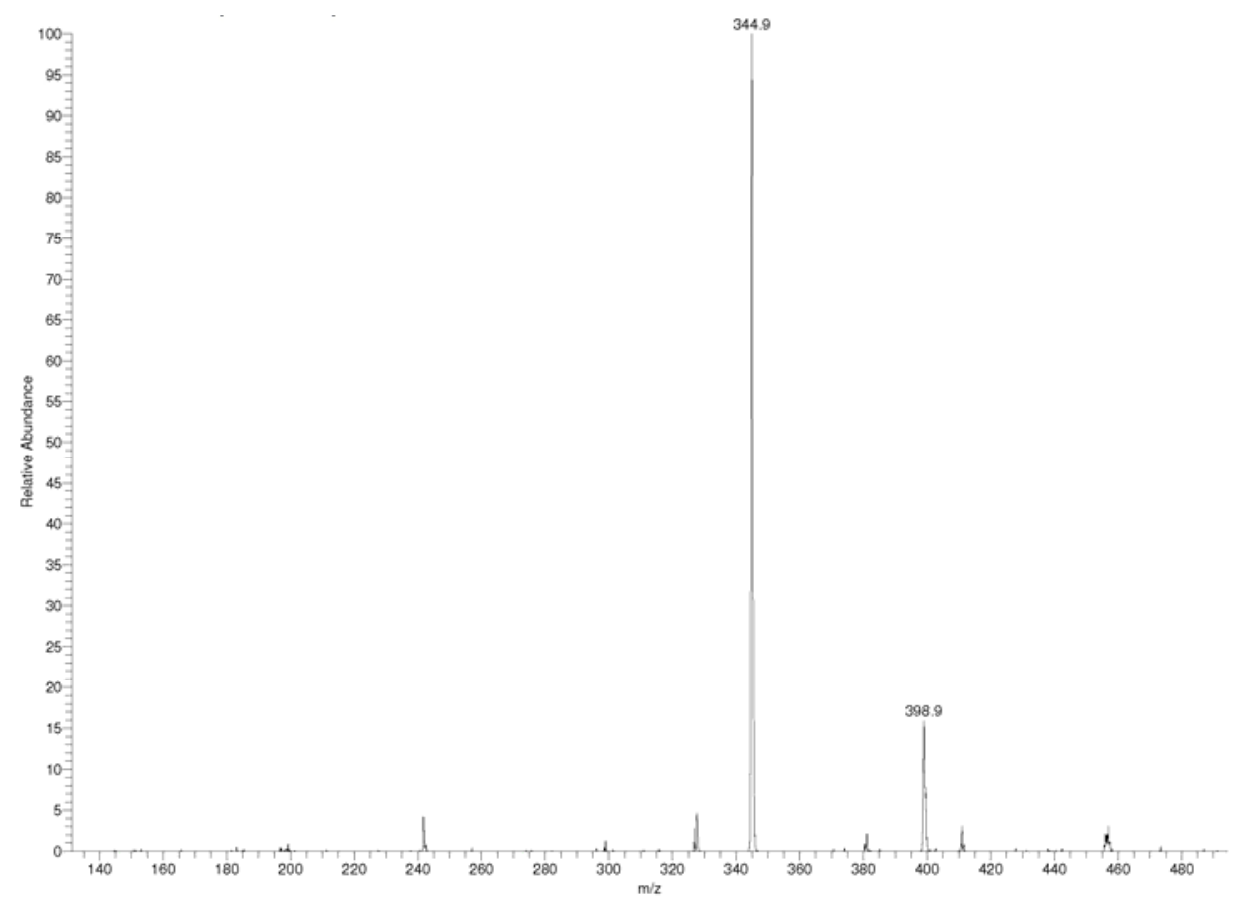

Figure 2.3.2 MS/MS of GS-DNB

EdAG was then synthesized from GS-DNB by addition of $0.5 \mathrm{M} \mathrm{NaOH}$ to induce a basecatalyzed $\beta$-elimination of the 1-S-(2,4-dinitrobenzene), followed by purification steps. Typical yields ranged from 5-20\%. The structure of EdAG was determined first by proton nuclear magnetic resonance $\left({ }^{1} \mathrm{H} N M R\right)$ in deuterium oxide $\left(\mathrm{D}_{2} \mathrm{O}\right)$, where the two vinylic protons of the dehydroalanine moiety resonate as a doublet at $5.76 \mathrm{ppm}$ (Figure 2.3.3). The resulting ${ }^{1} \mathrm{H}$ NMR spectrum is comparable to previous spectra that were fully characterized (29). 


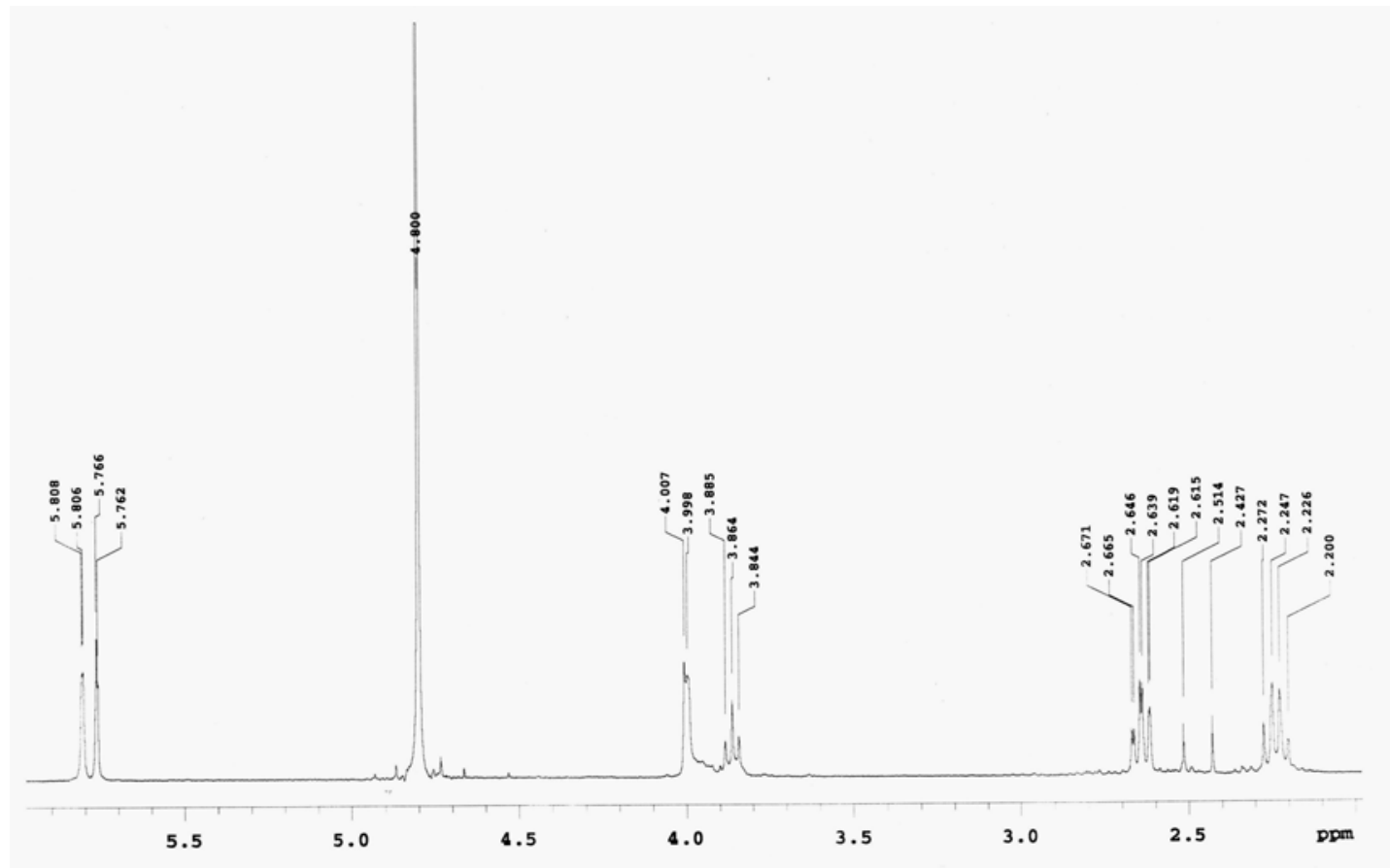

Figure 2.3.3 $\quad{ }^{1} \mathrm{H}$ NMR of EdAG

The purity of EdAG was determined by LC/MS/MS, where the presence of a single peak in both the $\mathrm{UV}_{254 \mathrm{~nm}}$ absorbance and the MS/MS spectrum suggested EdAG is the major constituent present (inset of Figure 2.3.4). Tandem mass spectrometric (MS/MS) structural confirmation was determined on the EdAG ion at m/z 274 that produced the daughter ions $m / z$ 145, m/z 199, and $m / z 256$, corresponding to the $\mathrm{MH}^{+}$EdAG molecular ion with neutral losses of pyroglutamic acid, glycine, and water, respectively (Figure 2.3.4). 


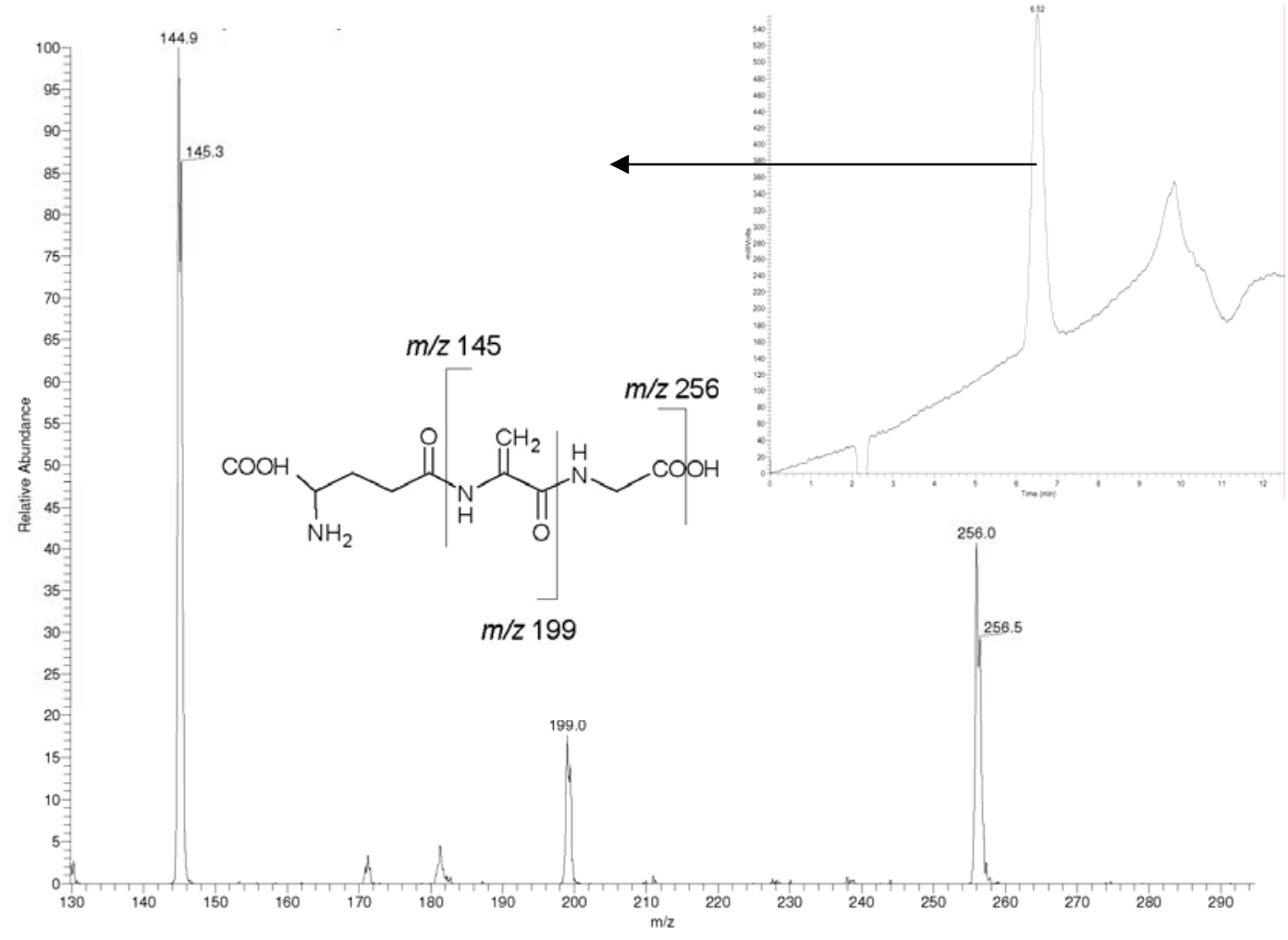

Figure 2.3.4 LC/MS/MS Identification of EdAG

\subsubsection{Formation of EdAG from S-Nitrosylated GSH (GSNO)}

EdAG synthesis could occur via an alternate mechanism by the potential loss of S-nitric oxide (S-NO) from S-nitrosylated glutathione (GSNO). S-Nitrothiols are stable carriers of nitric oxide (NO), of which S-nitrosylated glutathione is one of the most abundant. SNitrosoglutathione, or GSNO, is capable of transporting NO as well as transporting GSH to induce such reactions as protein S-nitrosylation and S-glutathionylation (38). S-Nitrosylated proteins are formed from transnitrosylation of NO from GSNO to cysteine residues on proteins. S-Glutathionylation of proteins could be formed from degradation of GSNO to EdAG, through the loss of S-NO, and subsequent conjugation of EdAG by a cysteine residue. The 
aforementioned chemical synthesis of EdAG involved removal of hydrophobic impurities resulting from the 1-S-(2,4-dinitrobenzene) moiety that has relatively poor yield. It was hypothesized that GSNO could lose S-NO to form EdAG under similar alkaline conditions in a much cleaner synthesis procedure with potential higher product yields. The stability of GSNO was determined by incubation for 24 hours in a $37^{\circ} \mathrm{C}$ water bath in Tris buffer, $\mathrm{pH} 8.4$, which were optimal degradation conditions for GSNO (39). More alkaline conditions $(\mathrm{pH}>9)$ resulted in poor mass spectra with the absence of any recognizable molecular ion. Three separate single ion monitoring scans were performed for GS-NEM, GSNO, and EdAG. No external EdAG was added, thus any EdAG detected was from GSNO degradation to EdAG and S-NO. Figure 2.3.5 displays the GSNO degradation after 24 hours, where a substantial amount of GSNO degraded to GSH, which was immediately trapped by NEM to form GS-NEM ( $\mathrm{m} / \mathrm{z} 433$; bottom panel). A very small, insignificant amount of GSNO degraded to EdAG ( $m / z$ 274; top panel), which demonstrated the inability of GSNO to be a reliable precursor for EdAG chemical synthesis on a large scale. The majority of GSNO remained stable after 24 hours ( $\mathrm{m} / \mathrm{z} 337$; middle panel). 


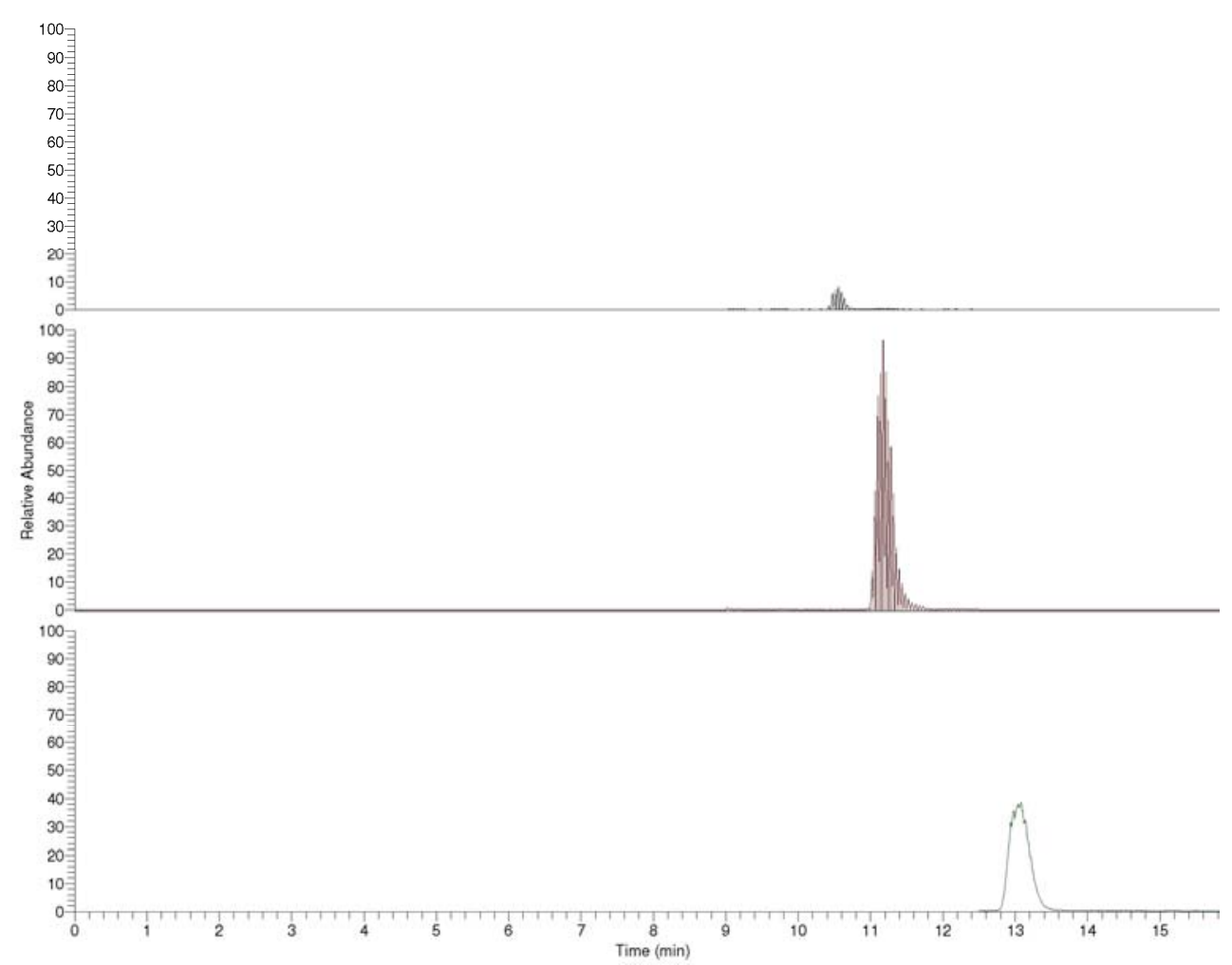

Figure 2.3.5 LC/MS of GSNO 24 hour degradation

\subsubsection{Identification of EdAG after in vitro Incubation of Busulfan with Human Liver}

\section{Cytosol}

A mass spectrometric detection method was developed to determine if EdAG is a metabolite of busulfan by human liver enzymes. Busulfan was incubated with GSH and human liver cytosol, which contains the major soluble isoforms of GSTs that would normally be present in hepatocytes, under physiological conditions of $\mathrm{pH} 7.4$ and $37^{\circ} \mathrm{C}$. The presence of busulfan metabolites was determined by LC/MS analysis after 2 and 24 hours. Figure 2.3.6 contains panels A, B, C, and D that monitored the molecular ions $\mathrm{m} / \mathrm{z} 308, \mathrm{~m} / \mathrm{z} 362, \mathrm{~m} / \mathrm{z} \mathrm{274}$, and $\mathrm{m} / \mathrm{z} 581$, corresponding to reduced GSH, GS ${ }^{+}$THT, EdAG, and GSG, respectively. Through two hours of 
incubation, busulfan-GSH conjugation catalyzed by GSTs formed the unstable sulfonium GS $^{+}$THT (Panel B) with excess GSH (Panel A) remaining in solution. The EdAG (Panel C) formed, resulting from spontaneous degradation of $\mathrm{GS}^{+} \mathrm{THT}$, was immediately conjugated through nucleophilic addition by GSH in solution to form the non-reducible thioether (or lanthionine) GSG (Panel D).

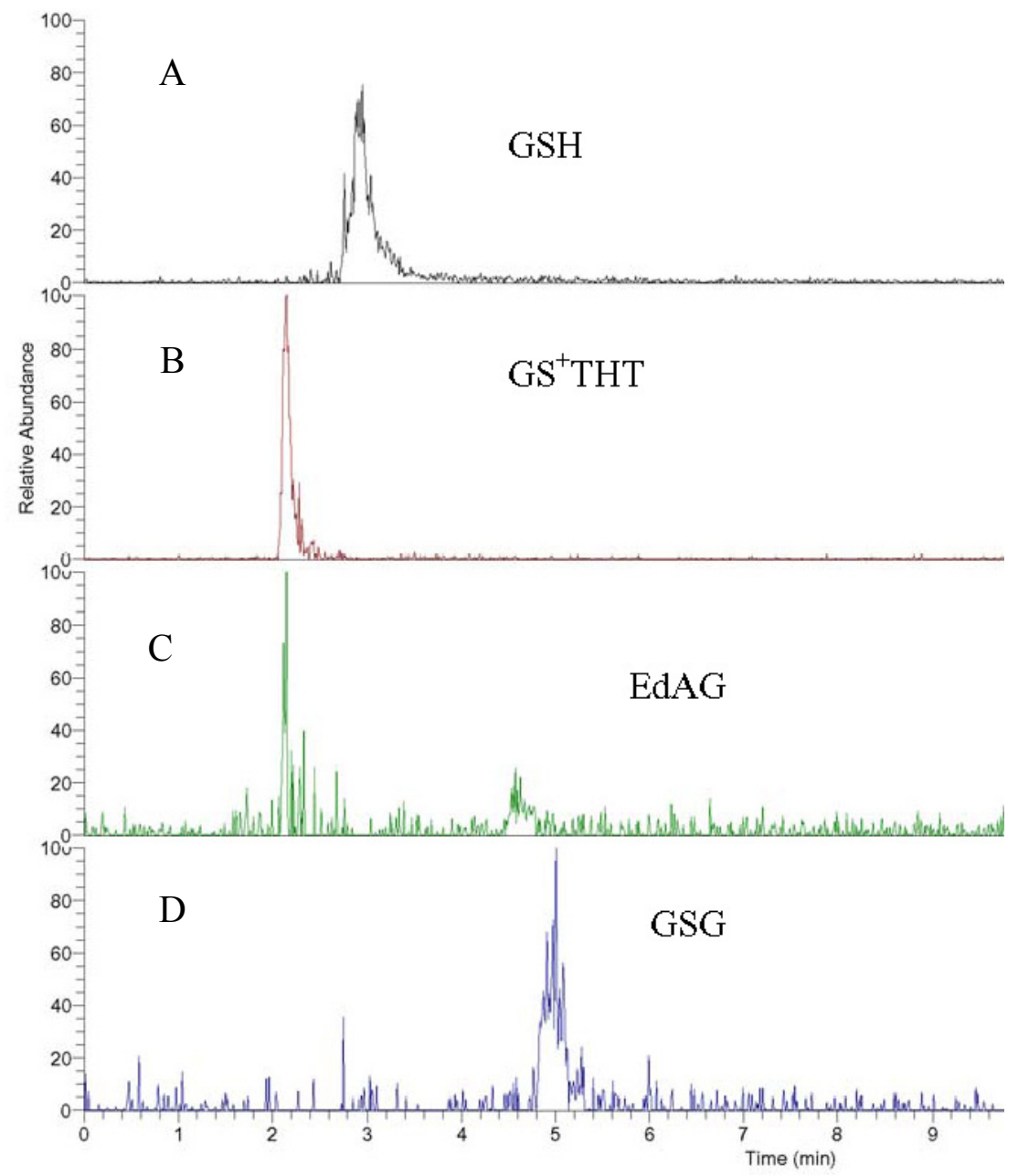

Figure 2.3.6 Busulfan Incubation with Human Liver Cytosol after 2 hours incubation 
Through the next 22 hours, the GSTs continued to conjugate busulfan with GSH to form $\mathrm{GS}^{+}$THT, which slowly degraded to EdAG at pH 7.4 and $37^{\circ} \mathrm{C}$. After 24 hours, the GSH in solution was depleted due to enzymatic busulfan conjugation, nonenzymatic conjugation of EdAG to form GSG, or oxidation to GSSG (not shown). Figure 2.3.7 demonstrates the depletion of GSH (Panel A), however GS ${ }^{+}$THT (Panel B) was still abundant because of its slow degradation at $\mathrm{pH}$ 7.4. $\mathrm{GS}^{+} \mathrm{THT}$ degraded much faster at $\mathrm{pH} 8.4$ than under physiological $\mathrm{pH}$ conditions as shown by previous work in our lab (29). Due to depletion of GSH and continual degradation of GS ${ }^{+}$THT to EdAG, EdAG (Panel C) was allowed to accumulate as there was little or no GSH to trap EdAG to form GSG (Panel D). EdAG was thus shown to be a busulfan metabolite from GST-catalyzed glutathione conjugation. 


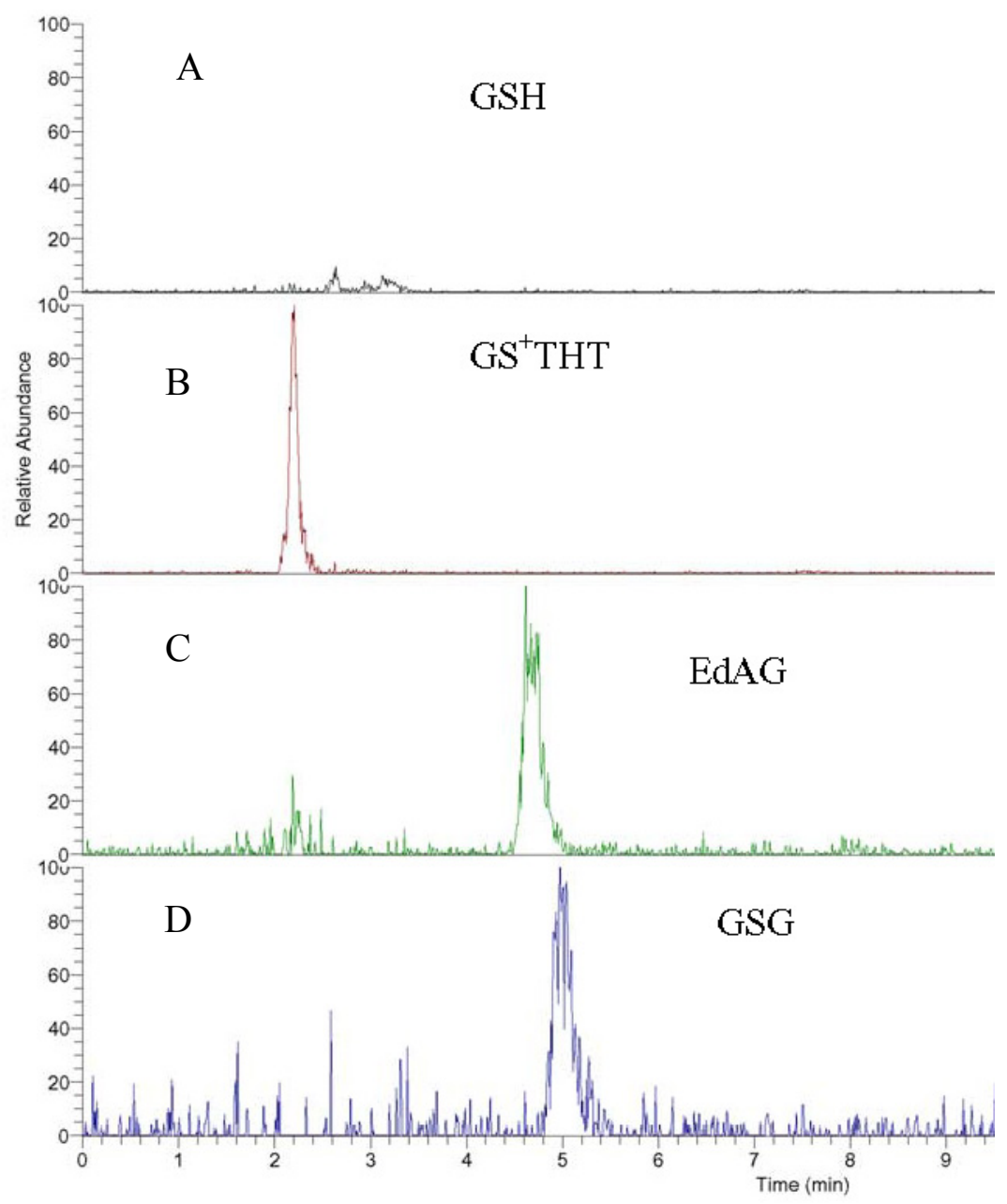

Figure 2.3.7 Busulfan Incubation with Human Liver Cytosol after 24 hours incubation 


\section{DISCUSSION}

\section{4}

Mass spectrometry is a powerful analytical tool with the ability to elucidate structural information from compounds. ESI is a soft ionization technique that allows molecules to stay intact for analysis of the molecular ion $\left(\mathrm{MH}^{+}\right)$. Ion trap mass analyzers have the ability to fragment the molecular ion through numerous cycles using multi-stage mass spectrometry $\left(\mathrm{MS}^{\mathrm{n}}\right)$, which can help elucidate the structure by studying neutral losses from the $\mathrm{MH}^{+}$and its product ions. This technology was useful for determining the structure and purity of the synthesized $\gamma$ glutamyldehydroalanylglycine (EdAG) from GSH and CDNB.

The first step in the synthesis of EdAG was the formation of the dinitrophenyl-Sconjugate of glutathione. This was performed by mixing equimolar solutions of GSH and 1chloro-2,4-dinitrobenzene (CDNB) slowly and providing ample time and mixing to ensure complete conjugation. This product was subsequently purified by precipitation from solution and recrystallization from hot water. This reaction is relatively efficient, with product yields typically ranging from 54-85\%. Caution must be taken in regards to contact and inhalation of CDNB and GS-DNB. In order to determine whether the bright yellow product was in fact GSDNB, ESI-MS/MS was employed to elucidate the structure. GS-DNB is insoluble in water, thus methanol was used to dissolve GS-DNB crystals with $0.1 \%$ formic acid. An intense signal at $\mathrm{m} / \mathrm{z}$ 474 appeared, corresponding to the molecular ion (Figure 2.3.1). Fragmentation of the $\mathrm{MH}^{+}$was performed using CID to study the product ions to ensure validity of the structure. Because GSDNB contains a GSH backbone consisting of glutamate, cysteine, and glycine residues, the typical neutral losses by CID associated with GSH were expected, such as the losses of water, 
glycine, and pyroglutamic acid. Indeed, fragmentation by CID of GS-DNB showed neutral losses of glycine and pyroglutamic acid (Figure 2.3.2). Pyroglutamic acid is a common neutral loss for GSH-containing compounds when analyzed by ESI-MS/MS $(40,41)$. This suggested that the major constituent in the bright yellow product was GS-DNB with a $\mathrm{MH}^{+}$of $m / z 474$.

From GS-DNB, EdAG was formed by adding strong base $(0.5 \mathrm{M} \mathrm{NaOH})$ to facilitate the $\beta$-elimination of 1-S-(2,4-dinitrobenzene) and leaving the tripeptide $\gamma$ glutamyldehydroalanylglycine, or EdAG. The bright yellow crystals dissolved in the base rapidly and the color of solution quickly turned to a dark amber and eventually to a deep orange/brown color over the $30 \mathrm{~min}$ stir. To remove the hydrophobic impurities responsible for the dark color, secondary butanol was added to extract the impurities from the aqueous solution of EdAG. The remaining aqueous layer was reduced in volume by approximately $50 \%$ by the slightly polar nature of sec-butanol and accounting, at least partly, for the poor product yield. The dilution of the aqueous layer to $300 \mathrm{~mL}$ with deionized water allowed for a more efficient ion exchange to occur by the Dowex ${ }^{\circledR} \mathrm{H}^{+} / \mathrm{Na}^{+}$exchange resin, which dropped the $\mathrm{pH}$ of the solution from 13 to 3. After evaporation, there was a light brown, water soluble solid. ${ }^{1} \mathrm{H}$ NMR and LC/MS/MS confirmed the structure of the major constituent of the product.

The ${ }^{1} \mathrm{H}$ NMR showed the presence of the two vinylic protons associated with the dehydroalanine moiety of EdAG, suggesting that EdAG was the major product formed. High performance liquid chromatography (HPLC) interfaced with tandem mass spectrometry (LC/MS/MS) was then performed to determine the presence of UV-detected impurities with a signal in the mass spectrum in the range of $m / z$ 100-1000. The UV chromatogram showed only one major peak at $254 \mathrm{~nm}$, which corresponded to EdAG at $\mathrm{m} / \mathrm{z} 274$ as indicated by the unique MS/MS fragmentation pattern corresponding to EdAG (Figure. 2.3.4). The mass spectrum did 
reveal other impurities present in the aqueous solution of product, particularly the sodium adduct of EdAG at $\mathrm{m} / \mathrm{z}$ 296. There were other ions present in the mass spectrum, but they were minor products compared to EdAG. It was concluded that EdAG was successfully and sufficiently synthesized for use in future experiments.

The anticancer agent busulfan is commonly used to treat chronic myelogenous leukemia $(2,3)$, polycythemia rubra vera (5), essential thrombocythemia (6), and in high dose therapy, coadministered with cyclophosphamide, as conditioning therapy prior to hematopoietic or bone marrow stem cell transplants (4). The major route of busulfan metabolism is glutathione conjugation catalyzed by GSTA1-1 in the cytosol of hepatocytes (27) to form the unstable sulfonium $\mathrm{GS}^{+} \mathrm{THT}$ as shown in Scheme 1.1. The sulfonium nonenzymatically degrades to THT (26) and the dehydroalanine analog of glutathione, EdAG (29). As long as GSH is present, electrophiles such as EdAG can be conjugated and detoxified. However during high dose busulfan administration, e.g. bone marrow transplant conditioning therapy, GSH can become depleted and render the cell vulnerable to reactive electrophiles. The amount of EdAG produced is directly proportional to the amount of metabolized busulfan, however that exact amount is nearly impossible to quantitate. It is assumed that intrahepatocellular concentrations of EdAG are not nearly as great as the millimolar concentrations of intracellular GSH. However the increased oxidative stress state of the liver could be associated with depletion of reduced GSH by oxidation to GSSG.

Busulfan has been shown to deplete intracellular hepatic GSH levels in vitro in murine models by as much as 50\% (31) and it is known that the major metabolite of the active form of the prodrug cyclophosphamide (4-hydroxycyclophosphamide) is detoxified by GSH, further depleting GSH. In myeloablative therapy, busulfan is normally administered first, followed by a 
washout period of at least 24 hours, then treatment with cyclophosphamide. Because both drugs require GSH for detoxification, it is logical that intracellular hepatic GSH could be reduced to levels similar to those of the electrophilic compounds requiring GSH conjugation. A major concern during this combination therapy is the potential onset of hepatic veno-occlusive disease (HVOD). HVOD occurs in zone 3 of the centrilobular region of the liver, which contains the highest hepatocyte concentrations of the drug metabolizing enzymes (DMEs) of cytochrome P450s and GSTs (42). As a busulfan metabolite formed from GSTs, EdAG could be contributing to HVOD by inhibiting GSTs, or trapping GSH directly, resulting in increased busulfan exposure. EdAG could also be contributing to HVOD by inhibiting GSTs producing more circulating busulfan as will be demonstrated in a later chapter.

After incubation of busulfan, GSH, and human liver cytosol under physiological conditions, EdAG was detected by LC/MS as a busulfan metabolite. After 2 hours, most EdAG formed was immediately trapped by unreacted GSH to form GSG. This observation is consistent with the nonenzymatic and spontaneous conversion of $\mathrm{GS}^{+} \mathrm{THT}$ to EdAG and supports the electrophilic nature of the dehydroalanine-containing EdAG intermediate. However, after 24 hours of continuous busulfan conjugation catalyzed by the cytosolic GSTs in human liver cytosol, reduced GSH levels became depleted and EdAG was continually being formed by spontaneous degradation of GS ${ }^{+}$THT. With limited GSH reserves to form GSG, EdAG accumulated. This evidence suggested that EdAG is a busulfan metabolite from in vitro metabolism. Mass spectrometry has been known to form dehydroalanine residues from cysteines and serines through CID prior to the ion hitting the mass analyzer (43). The evidence of the accumulation of EdAG after 24 hours incubation suggests that the EdAG detected by LC/MS 
was not a byproduct of inadvertent mass spectrometric CID, but rather EdAG formation in solution by degradation of GS ${ }^{+}$THT to EdAG and THT.

In conclusion, this dissertation work has thus far demonstrated that EdAG can be successfully synthesized from a conjugate of glutathione (GS-DNB) and that EdAG is an in vitro busulfan metabolite. The next chapter will study the reactivity of EdAG towards cellular thiols. The amino acid L-cysteine, the cysteine-containing tripeptide glutathione (GSH), and protein models (bovine insulin and serum albumin) containing reactive cysteine residues will be incubated with EdAG to determine the extent of conjugation 


\section{REFERENCES}

(1) Ponti, M., Souhami, R. L., Fox, B. W., and Hartley, J. A. (1991) DNA interstrand crosslinking and sequence selectivity of dimethanesulphonates. Br. J. Cancer 63(5), 743-747.

(2) Silver, R. T. (2003) Chronic myeloid leukemia. Hematol. Oncol. Clin. North Am. 17(5), 1159-1vii.

(3) Stone, R. M. (2004) Optimizing treatment of chronic myeloid leukemia: a rational approach. Oncologist. 9(3), 259-270.

(4) Russell, S., and Vowels, M. (1992) Busulphan, cyclophosphamide, and melphalan as conditioning therapy in allogeneic bone marrow transplants for acute lymphoblastic leukemia. Transplant. Proc. 24(1), 183.

(5) Mital, A., Czyz, J., and Hellmann, A. (1996) Evaluation of treatment results in patients with polycythemia rubra vera. Pol. Arch. Med. Wewn. 95(5), 453-458.

(6) Van de Pette, J. E., Prochazka, A. V., Pearson, T. C., Singh, A. K., Dickson, E. R., and Wetherley-Mein, G. (1986) Primary thrombocythaemia treated with busulphan. Br. J. Haematol. 62(2), 229-237.

(7) Leger, F., Nguyen, L., and Puozzo, C. (2009) Exposure equivalence between IV (0.8 $\mathrm{mg} / \mathrm{kg})$ and oral $(1 \mathrm{mg} / \mathrm{kg})$ busulfan in adult patients. Eur. J. Clin. Pharmacol.

(8) Russell, J. A., and Kangarloo, S. B. (2008) Therapeutic drug monitoring of busulfan in transplantation. Curr. Pharm. Des 14(20), 1936-1949.

(9) Grochow, L. B., Jones, R. J., Brundrett, R. B., Braine, H. G., Chen, T. L., Saral, R., Santos, G. W., and Colvin, O. M. (1989) Pharmacokinetics of busulfan: 
correlation with veno-occlusive disease in patients undergoing bone marrow transplantation. Cancer Chemother. Pharmacol. 25(1), 55-61.

(10) Grochow, L. B., Krivit, W., Whitley, C. B., and Blazar, B. (1990) Busulfan disposition in children. Blood 75(8), 1723-1727.

(11) Hassan, M., Oberg, G., Bekassy, A. N., Aschan, J., Ehrsson, H., Ljungman, P., Lonnerholm, G., Smedmyr, B., Taube, A., Wallin, I., and . (1991) Pharmacokinetics of high-dose busulphan in relation to age and chronopharmacology. Cancer Chemother. Pharmacol. 28(2), 130-134.

(12) Hassan, M., Ljungman, P., Bolme, P., Ringden, O., Syruckova, Z., Bekassy, A., Stary, J., Wallin, I., and Kallberg, N. (1994) Busulfan bioavailability. Blood 84(7), 21442150.

(13) Ehrsson, H., Hassan, M., Ehrnebo, M., and Beran, M. (1983) Busulfan kinetics. Clin. Pharmacol. Ther. 34(1), 86-89.

(14) Sandstrom, M., Karlsson, M. O., Ljungman, P., Hassan, Z., Jonsson, E. N., Nilsson, C., Ringden, O., Oberg, G., Bekassy, A., and Hassan, M. (2001) Population pharmacokinetic analysis resulting in a tool for dose individualization of busulphan in bone marrow transplantation recipients. Bone Marrow Transplant. 28(7), 657-664.

(15) Tran, H. T., Madden, T., Petropoulos, D., Worth, L. L., Felix, E. A., Sprigg-Saenz, H. A., Choroszy, M., Danielson, M., Przepiorka, D., and Chan, K. W. (2000) Individualizing high-dose oral busulfan: prospective dose adjustment in a pediatric population undergoing allogeneic stem cell transplantation for advanced hematologic malignancies. Bone Marrow Transplant. 26(5), 463-470. 
(16) Petros, W. P., and Evans, W. E. (2005) Anticancer Agents. In Applied Pharmacokinetics and Pharmacodynamics: Principles of Therapeutic Drug Monitoring (Burton, M. E., Shaw, L. M., Schentag, J. J., and Evans, W. E., Eds.) pp 617-636, Lippincott Williams and Wilkins,

(17) Bertholle-Bonnet, V., Bleyzac, N., Galambrun, C., Mialou, V., Bertrand, Y., Souillet, G., and Aulagner, G. (2007) Influence of underlying disease on busulfan disposition in pediatric bone marrow transplant recipients: a nonparametric population pharmacokinetic study. Ther. Drug Monit. 29(2), 177-184.

(18) Poonkuzhali, B., Srivastava, A., Quernin, M. H., Dennison, D., Aigrain, E. J., Kanagasabapathy, A. S., Krishnamoorthy, R., and Chandy, M. (1999) Pharmacokinetics of oral busulphan in children with beta thalassaemia major undergoing allogeneic bone marrow transplantation. Bone Marrow Transplant. 24(1), 5-11.

(19) Tran, H., Petropoulos, D., Worth, L., Mullen, C. A., Madden, T., Andersson, B., Choroszy, M., Nguyen, J., Webb, S. K., and Chan, K. W. (2004) Pharmacokinetics and individualized dose adjustment of intravenous busulfan in children with advanced hematologic malignancies undergoing allogeneic stem cell transplantation. Biol. Blood Marrow Transplant. 10(11), 805-812.

(20) Gibbs, J. P., Gooley, T., Corneau, B., Murray, G., Stewart, P., Appelbaum, F. R., and Slattery, J. T. (1999) The impact of obesity and disease on busulfan oral clearance in adults. Blood 93(12), 4436-4440.

(21) Ehrsson, H., and Hassan, M. (1984) Binding of busulfan to plasma proteins and blood cells. J. Pharm. Pharmacol. 36(10), 694-696. 
(22) Hassan, M., Ehrsson, H., and Ljungman, P. (1996) Aspects concerning busulfan pharmacokinetics and bioavailability. Leuk. Lymphoma 22(5-6), 395-407.

(23) Hassan, M., Ehrsson, H., Smedmyr, B., Totterman, T., Wallin, I., Oberg, G., and Simonsson, B. (1989) Cerebrospinal fluid and plasma concentrations of busulfan during high-dose therapy. Bone Marrow Transplant. 4(1), 113-114.

(24) Hassan, M., Oberg, G., Ehrsson, H., Ehrnebo, M., Wallin, I., Smedmyr, B., Totterman, T., Eksborg, S., and Simonsson, B. (1989) Pharmacokinetic and metabolic studies of high-dose busulphan in adults. Eur. J. Clin. Pharmacol. 36(5), 525-530.

(25) Vassal, G., Gouyette, A., Hartmann, O., Pico, J. L., and Lemerle, J. (1989) Pharmacokinetics of high-dose busulfan in children. Cancer Chemother. Pharmacol. 24(6), 386-390.

(26) Roberts, J. J., and Warwick, G. P. (1961) The mode of action of alkylating agents. II. Studies of the metabolism of myleran. The reaction of myleran with some naturally occurring thiols in vitro. Biochem. Pharmacol. 6, 205-216.

(27) Czerwinski, M., Gibbs, J. P., and Slattery, J. T. (1996) Busulfan conjugation by glutathione S-transferases alpha, mu, and pi. Drug Metab Dispos. 24(9), 10151019.

(28) Hassan, M., and Ehrsson, H. (1987) Urinary metabolites of busulfan in the rat. Drug Metab Dispos. 15(3), 399-402.

(29) Younis, I. R., Elliott, M., Peer, C. J., Cooper, A. J., Pinto, J. T., Konat, G. W., Kraszpulski, M., Petros, W. P., and Callery, P. S. (2008) Dehydroalanine analog of glutathione: an electrophilic busulfan metabolite that binds to human glutathione S-transferase A1-1. J. Pharmacol. Exp. Ther. 327(3), 770-776. 
(30) Bouligand, J., Boland, I., Valteau-Couanet, D., Deroussent, A., Kalifa, C., Hartmann, O., and Vassal, G. (2003) In children and adolescents, the pharmacodynamics of high-dose busulfan is dependent on the second alkylating agent used in the combined regimen (melphalan or thiotepa). Bone Marrow Transplant. 32(10), 979-986.

(31) DeLeve, L. D., and Wang, X. (2000) Role of oxidative stress and glutathione in busulfan toxicity in cultured murine hepatocytes. Pharmacology 60(3), 143-154.

(32) Srivastava, A., Poonkuzhali, B., Shaji, R. V., George, B., Mathews, V., Chandy, M., and Krishnamoorthy, R. (2004) Glutathione S-transferase M1 polymorphism: a risk factor for hepatic venoocclusive disease in bone marrow transplantation. Blood 104(5), 1574-1577.

(33) Kebarle, P. (2000) A brief overview of the present status of the mechanisms involved in electrospray mass spectrometry. J. Mass Spectrom. 35(7), 804-817.

(34) Fenn, J. B., Mann, M., Meng, C. K., Wong, S. F., and Whitehouse, C. M. (1989) Electrospray ionization for mass spectrometry of large biomolecules. Science 246(4926), 64-71.

(35) Fenn, J. (2002) Electrospray ionization mass spectrometry: How it all began. J. Biomol. Tech. 13(3), 101-118.

(36) Sokolovsky, M., Sadeh, T., and Patchornik, A. (1963) Nonenzymatic cleavages of peptide chains at the cysteine and serine residues through their conversion to dehydralanine (DHAL). II. The specific chemical cleavage of cysteinyl peptides. J. Am. Chem. Soc. 86(6), 1212-1217. 
(37) Asquith, R. S., and Carthew, P. (1972) The preparation and subsequent identification of a dehydroalanyl peptide from alkali-treated oxidised glutathione. Biochim. Biophys. Acta 285(2), 346-351.

(38) Ji, Y., Akerboom, T. P., Sies, H., and Thomas, J. A. (1999) S-nitrosylation and Sglutathiolation of protein sulfhydryls by S-nitroso glutathione. Arch. Biochem. Biophys. 362(1), 67-78.

(39) Heikal, L., Martin, G. P., and Dailey, L. A. (2009) Characterisation of the decomposition behaviour of S-nitrosoglutathione and a new class of analogues: SNitrosophytochelatins. Nitric. Oxide. 20(3), 157-165.

(40) Castro-Perez, J., Plumb, R., Liang, L., and Yang, E. (2005) A high-throughput liquid chromatography/tandem mass spectrometry method for screening glutathione conjugates using exact mass neutral loss acquisition. Rapid Commun. Mass Spectrom. 19(6), 798-804.

(41) Zhu, Y., Gieselman, M. D., Zhou, H., Averin, O., and van der Donk, W. A. (2003) Biomimetic studies on the mechanism of stereoselective lanthionine formation. Org. Biomol. Chem. 1(19), 3304-3315.

(42) el, M. M., and Kauffman, F. C. (1986) Sublobular distribution of transferases and hydrolases associated with glucuronide, sulfate and glutathione conjugation in human liver. Hepatology 6(3), 450-456.

(43) Thakur, S. S., and Balaram, P. (2008) Fragmentation of peptide disulfides under conditions of negative ion mass spectrometry: studies of oxidized glutathione and contryphan. J. Am. Soc. Mass Spectrom. 19(3), 358-366. 


\section{Chapter 3}

Conjugation addition reactions of the

dehydroalanine analog of GSH toward cellular

thiols 


\section{INTRODUCTION}

\subsection{Reactivity of Dehydroalanines}

\subsubsection{EdAG is a dehydroalanine analog of Glutathione}

Glutathione (GSH) is the most prevalent low molecular weight cellular thiol in mammalian cells. In addition to a major cellular role in oxidative status, there is an important function of GSH in sequestering toxic, electrophilic xenobiotics (1). Nucleophilic reactivity of GSH is based on conjugation of its thiol group with electrophilic compounds (2). Conjugation with GSH can either be nonenzymatic nucleophilic addition or catalyzed by the glutathione-Stransferase (GST) superfamily of enzymes. There are numerous isoenzymes of GSTs, classified into cytosolic (soluble), membrane, or mitochondrial. There are eight major families of mammalian GSTs (alpha, zeta, theta, kappa, mu, pi, sigma, and omega) (3). Dehydroalanine (Dha) residues are formed posttranslationally mostly by a $\beta$-elimination of serine and threonine residues, but also occurs less readily on cysteine residues. Dha-containing peptides react as Michael acceptors that link cysteine residues with dehydroalanine groups to generate nonreducible thioethers as a posttranslational modification (4).

\subsubsection{Reactivity of Dehydroalanine Moieties within Proteins}

Dehydroalanine residues are common post-translational modifications within many proteins, not just lantibiotics. In these settings however, the presence of Dha residues are most likely a sign of oxidation or damage such that the protein is no longer functional. For dehydroalanines to be formed within proteins, either serine/threonine or cysteine residues must be dehydroxylated or dethiolated, respectively. This occurs most frequently during food 
processing under the intense heat and alkali conditions that favor the $\beta$-elimination of cysteine/serine side chains to form the Dha. Also, an in situ alkaline condition (i.e. nearby basic amino acids) can also promote the $\beta$-elimination to Dha. The cysteine-to-Dha transformation has been studied in vitro in somatostatin, insulin, and transthyretin $(5,6)$. When proteins are studied by mass spectrometry, the formation of Dha could be caused by instrument itself and essentially be artefactual of Dha formation in vivo (7).

One common plasma protein that is heavily involved in many physiological processes that Dha formation occurs on is human serum albumin (HSA). HSA is a protein that contains 35 cysteine residues, 34 of which are involved in disulfide bonds leaving one free cysteine at residue position 34. Human serum albumin has been studied to determine whether the any Cysto-Dha transformation is the result of a post-translational modification in vivo, the result of food processing, or some other ex vivo factor such as incidental collision-induced dissociation of the cysteine thiol side chain in the mass spectrometer. The existence of the Cys487-to -Dha biotransformation on both commercially-purchased HSA and plasma HSA confirmed that the $\beta$ elimination occurred as a post-translational modification in vivo (8). The presence of a Dha on residue position 487 of HSA is indicative of the biotransformation of Cys487-to-Dha and the oxidation of the protein, which makes it vulnerable to degradation by proteinases because of the changes in secondary and tertiary protein structure (9).

Another set of proteins in which dehydroalanine residues are present are human lens proteins. It has been demonstrated that Dha residues exist in many water soluble and insoluble human lens proteins $(10,11)$. The formation of Dha residues originated from either cysteines or serines (phosphoserines) that underwent a hydroxide-mediated $\beta$-elimination $(4,11,12)$. Aged lens proteins tend to have more Dha residues than younger lens proteins. When there are nearby 
cysteines, histidines, or lysines within the same protein or adjacent proteins, lanthionines, histidinoalanines, and lysinoalanines form, respectively (13). The formation of lanthionines are favored not because of the presence of more cysteines than histidines or lysines, but the presence of glutathione within the human lens (up to $2 \mathrm{mM}$ ) (14). GSH can form disulfide bonds (R-S-SR) with existing cysteine residues on proteins or non-reducible thioethers (R-S-R) with Dha residues, both of which could result in the aggregation of proteins that lead to cataracts, which are more prominent in aged lens proteins because of the incidence of Dha residues $(10,15)$.

There are numerous other examples of Dha residues within specific proteins that play a role in altering that protein's function, such as casein, thiostrepton, human plasma mannanbinding lectin, and histidine ammonia lyase (16-19). Dehydroalanines have also been synthesized within peptides to utilize their reactive properties to study protein interactions or to act as inhibitors $(4,12,20-31)$.

\subsubsection{Chapter Goals}

It has previously been demonstrated that EdAG is a busulfan metabolite from an in vitro phase II glutathione conjugation reaction catalyzed by soluble GSTs in human liver cytosol. It is desired in this chapter to study the reactivity of the electrophilic EdAG towards cellular nucleophiles such as GSH, L-cysteine, and cysteine residues in proteins. Nucleophilic addition of these thiol-containing nucleophiles with the electrophilic EdAG has the potential to form the non-reducible lanthionine, or thioether, bridge (R-S-R). 


\section{EXPERIMENTAL PROCEDURES}

\subsubsection{Materials}

L-Cysteine, formic acid, GSH, and Tris buffer, $\mathrm{pH}$ 7.4, tris-(2-carboxyethyl)phosphine (TCEP), bovine insulin, bovine serum albumin (BSA), $\alpha$-cyano-4-hydroxycinnamic acid, and trypsin were purchased from Sigma-Aldrich (St. Louis, MO). Sodium bicarbonate, methanol (optima), acetonitrile (optima), isopropyl alcohol, and hydrochloric acid were purchased from Fisher Scientific (Pittsburgh, PA). Pooled human liver cytosol (HLC) from multiple donors, containing soluble isoforms of GSTs $(20 \mathrm{mg} / \mathrm{mL}$ total protein concentration) was purchased from BD Gentest (Bedford, MA). All water used was deionized with a NANOpure ${ }^{\circledR}$ water purification system (Barnstead, San Jose, CA).

\subsubsection{Instrumentation}

High-performance liquid chromatography (HPLC) was performed on a Shimadzu 10 Avp binary pump HPLC system equipped with a UV/Vis detector (Shimadzu Scientific Instruments, Columbia, MD). Mass spectrometry was performed using an electrospray ionization (ESI) quadrupole ion trap (QIT) LCQ DECA (ThermoFisher Scientific, Waltham, MA), capable of

multi-stage mass spectrometry (MS, MS/MS, $\mathrm{MS}^{3}$ ). Matrix-assisted laser desorption ionizationtime of flight (MALDI-TOF) mass spectrometric analyses were performed on a Micromass Reflectron MALDI-TOF, (Waters, Milford, MA). 


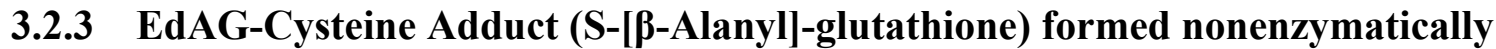

A solution of EdAG in water $(1.0 \mathrm{mM})$ was mixed with a solution of L-cysteine $(1.0 \mathrm{mM}$ in $100 \mathrm{mM}$ Tris buffer, $\mathrm{pH}$ 7.4) in $100 \mathrm{mM}$ Tris buffer, $\mathrm{pH} 7.4$ (final volume, $1.0 \mathrm{ml}$ ). The mixture was heated in a $37{ }^{\circ} \mathrm{C}$ water bath for 12 hours, with aliquots taken every hour to monitor the reaction. Aliquots were further diluted 200 -fold in a solution of $0.1 \%$ formic acid in methanol/water $(50: 50, \mathrm{v} / \mathrm{v})$, and the product was detected by electrospray mass spectrometry (ESI-MS). The mass spectrum of the predicted protonated molecular species of $m / z 395$ was consistent with the molecular weight of the cysteine-EdAG conjugate ( $S$-[ $\beta$-alanyl]-glutathione). MS/MS fragmentation of this conjugate was performed under the CID conditions of $30 \%$ collision energy and 1.5 amu window width. Further fragmentation of the major MS/MS production was subjected to MS/MS/MS $\left(\mathrm{MS}^{3}\right)$ with a collision energy of $40 \%$ and $1.5 \mathrm{amu}$ window width.

\subsubsection{Conjugate Addition of EdAG with Glutathione}

GSH (1.0 mM in $100 \mathrm{mM}$ Tris buffer, $\mathrm{pH}$ 7.4) was mixed with an aqueous solution of EdAG $(1.0 \mathrm{mM})$ in $100 \mathrm{mM}$ Tris buffer, $\mathrm{pH} 7.4$, and the mixture was incubated in a $37^{\circ} \mathrm{C}$ water bath overnight to provide the GSH-EdAG adduct (GSG). Identification of GSG was structurally determined by mass spectral analysis. The mass spectrum of the predicted component in the reaction mixture should give a protonated molecular species ion at $\mathrm{m} / \mathrm{z} 581$, consistent with molecular mass of the GSH-EdAG (GSG) conjugate. Tandem MS fragmentation was performed on the $\mathrm{MH}^{+}$ion of GSG in an ion trap mass spectrometer using a collision energy of $30 \%$ and a $1.5 \mathrm{amu}$ window width. 


\subsubsection{EdAG-Glutathione conjugation catalyzed by GSTs}

GSH (1.0 mM in $100 \mathrm{mM}$ Tris buffer, pH 7.4) was mixed with an aqueous solution of EdAG $(1.0 \mathrm{mM})$ in $100 \mathrm{mM}$ Tris buffer, $\mathrm{pH} 7.4$, and the mixture was incubated in a $37^{\circ} \mathrm{C}$ water bath for $12 \mathrm{~h}$ in the presence of $0.2 \mathrm{mg}$ of human liver cytosol, which contains all soluble isoforms of GSTs. Identification of GSG was made by LC/MS analysis and a qualitative estimate of increased GSG abundance was determined from comparison of controls without enzymes versus with enzymes. Chromatographic separation of the polar compounds was achieved using a Thermo Hypercarb column (100 x $2.1 \mathrm{~mm}$ I.D., $5 \mu \mathrm{m}$ particle size $)$

(ThermoFisher, Waltham, MA). A gradient of mobile phase A ( $0.5 \%$ formic acid in $90 / 10, \mathrm{v} / \mathrm{v}$, water/acetonitrile) and mobile phase B ( $0.1 \%$ formic acid in 50/50, v/v, isopropanol/methanol) at $0.2 \mathrm{~mL} / \mathrm{min}$ was run from $0-5$ minutes using $100 \% \mathrm{~A}$; from $5-7$ minutes, $0 \rightarrow 50 \% \mathrm{~B}$; from $7-9$ minutes, $50 \rightarrow 0 \% \mathrm{~B}$; and from 9-15 minutes, $100 \% \mathrm{~A}$. The mass spectrometer was operated in the positive ion full scan mode with a spray voltage of $5.2 \mathrm{kV}$, capillary voltage of $30 \mathrm{~V}$, capillary temperature of $300^{\circ} \mathrm{C}$, and a nitrogen sheath gas flow rate of $80 \mathrm{arb}$. units. The following ions were monitored: $\mathrm{m} / \mathrm{z} 274, \mathrm{~m} / \mathrm{z} 308$, and $\mathrm{m} / \mathrm{z} 581$, corresponding to EdAG, reduced GSH, GSG, respectively. The signal intensity of GSG formed nonenzymatically was compared to that of GSG formed in the presence of GSTs to determine if GSG conjugation was significantly enhanced by catalysis by GSTs.

\subsubsection{Reactivity of EdAG with Insulin}

Bovine insulin (MW $5734 \mathrm{Da}$ ) consists of an A-chain and a B-chain that is connected by two inter-chain disulfide bonds. The insulin was reduced to the B-chain (MW $3400 \mathrm{Da}$ ), containing two reactive thiols, by addition of $5 \mathrm{mM}$ tris-(2-carboxyethyl)phosphine (TCEP) to a 
$2 \mu \mathrm{M}$ solution of bovine insulin in deionized $\mathrm{H}_{2} \mathrm{O}, \mathrm{pH} 2.0$ (adjusted with $1 \mathrm{M} \mathrm{HCl}$ ). The reduction to the B-chain was complete within 2 hours and was monitored by both ESI-MS and MALDI-TOF. As a positive control, the B-chain was then incubated with the electrophilic Michael acceptor N-ethyl maleimide (NEM; MW $125 \mathrm{amu}$ ) to ensure that the two cysteine thiols are reactive toward electrophiles with an expected MW of $3650 \mathrm{Da}$. Excess NEM (1 mM) was used to ensure conjugation to both cysteine residues on reduced B-chain. Excess EdAG (1 mM) was then incubated with unreacted B-chain to determine the extent of EdAG reactivity towards cysteine residues on proteins, with an expected product of $3946 \mathrm{Da}$.

\subsubsection{Reactivity of EdAG with Albumin}

EdAG was reacted with a more biologically-relevant protein, serum albumin, to study the reactivity toward the free cysteine residue. Bovine serum albumin (BSA) was used in lieu of human serum albumin due to its availability and alkylation to both NEM and EdAG was performed based on a modified method of Li et al (32). BSA (60 $\mu \mathrm{M})$, containing 17 disulfide bonds, was reduced with $50 \mathrm{mM}$ TCEP and diluted to a volume of $500 \mu \mathrm{L}$ with $100 \mathrm{mM}$ Tris buffer, $\mathrm{pH} 7.4$, for 2.5 hours in a $55^{\circ} \mathrm{C}$ water bath. After cooling, excess NEM or EdAG (400 $\mathrm{mM}$ ) was added to reduced BSA for 2 hours, followed by filtration through Microcon $10 \mathrm{kDa}$ filters to remove excess TCEP and NEM or EdAG. Alkylated BSA was reconstituted with 500 $\mu \mathrm{L}$ of $100 \mathrm{mM} \mathrm{NH}_{4} \mathrm{HCO}_{3}$ buffer, $\mathrm{pH} 8.6$ and a 1/20 (trypsin/BSA, w/w) amount of trypsin was added to digest the alkylated BSA while incubated overnight in a $37 \mathrm{C}$ water bath. The digestion was quenched by the addition of $20 \%$ acetic acid. The expected BSA peptides from trypsin digestion were determined from a protein BLAST search and entered into a spreadsheet. The 
peptides containing cysteine residues were added to the mass of either NEM or EdAG to account for the expected MW of the modified residue. Many peptides contained more than one cysteine residue. MALDI-TOF mass spectral analyses were performed by mixing the digest solution with a solution of $10 \mathrm{mg} / \mathrm{mL} \alpha$-cyano-4-hydroxycinnamic acid in $0.1 \%$ formic acid in 90/10 (v/v) $\mathrm{ACN} / \mathrm{H}_{2} \mathrm{O}$ in a 1:1 manner $(\mathrm{v} / \mathrm{v})$. MALDI-TOF analyses were performed in the positive ion mode with a full scan range of $m / z 500-4000$. 


\section{RESULTS}

\subsubsection{EdAG-Cysteine Adduct (S-[B-Alanyl]-glutathione) formed nonenzymatically}

Equimolar amounts of reduced L-cysteine and EdAG were incubated in Tris buffer, $\mathrm{pH}$

7.4, and monitored by mass spectrometry to determine the extent of conjugation and the structure of that adduct. A molecular ion at $m / z 395$ was found, which corresponded to the EdAGcysteine, or S-( $\beta$-alanyl)-glutathione, adduct. Tandem mass spectrometry was performed on $\mathrm{m} / \mathrm{z}$ 395 and the major product ion formed was $\mathrm{m} / \mathrm{z} 266$, which corresponds to loss of pyroglutamic acid, a typical neutral loss in collision-induced dissociation of glutathione-like compounds (Figure 3.3.1). Minor product ions were formed from $\mathrm{m} / \mathrm{z} 266$ using multi-stage MS (MS/MS/MS, or $\mathrm{MS}^{3}$ ) at $\mathrm{m} / \mathrm{z} 248$ and $\mathrm{m} / \mathrm{z}$ 191, corresponding to neutral loss of water and glycine, respectively, from the ion at $\mathrm{m} / \mathrm{z} 266$.

Because EdAG is achiral due to the 1,4-Michael conjugated system containing the dehydroalanine moiety, the addition of nucleophiles such as GSH or L-cysteine to EdAG forms a new chiral center. The probability exists that GSG consists of a pair of diastereomers, however the stereochemistry of synthesized GSG and S-( $\beta$-alanyl)-glutathione was not determined. 


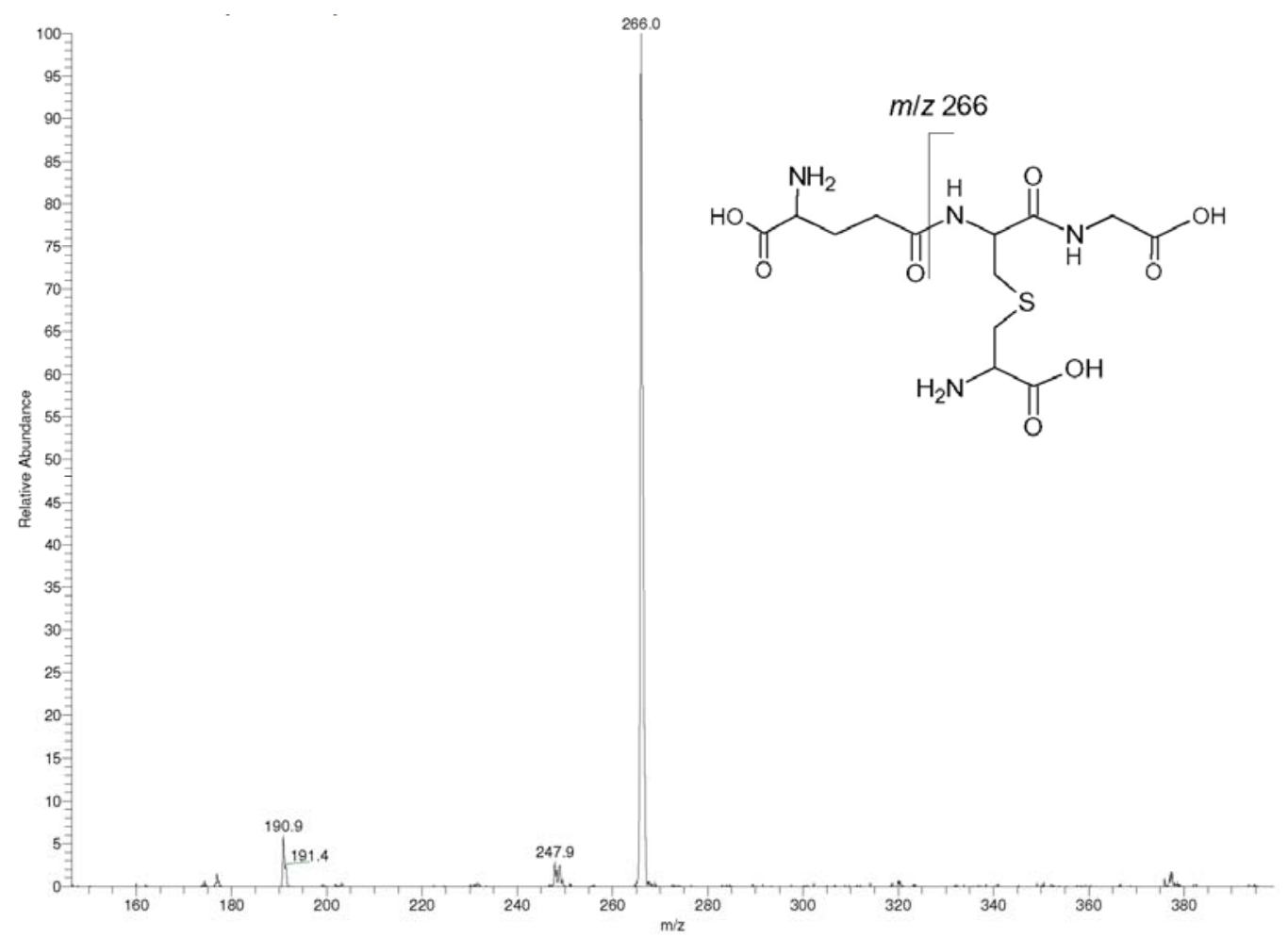

Figure 3.3.1 MS/MS Fragmentation Pattern of S-( $\beta$-Alanyl)-Glutathione

\subsubsection{Conjugate Addition of EdAG with Glutathione}

EdAG contains an $\alpha, \beta$-unsaturated carbonyl system that adds nucleophiles, such as sulfhydryl anions, to form a conjugate or adduct. Condensation of EdAG with the most abundant cellular thiol GSH was performed under physiological conditions and yielded a glutathione dimer analog with a non-reducible lanthionine cross-linkage (GSG). Tandem mass spectrometry was performed on the GSG molecular ion $\mathrm{m} / \mathrm{z} 581$ to confirm the structure (Figure 3.3.2), where the product ions formed at $\mathrm{m} / \mathrm{z} 323, \mathrm{~m} / \mathrm{z} 452$, and $\mathrm{m} / \mathrm{z} 563$ were from neutral losses of two pyroglutamic acid molecules, one pyroglutamic acid, and water, respectively. 


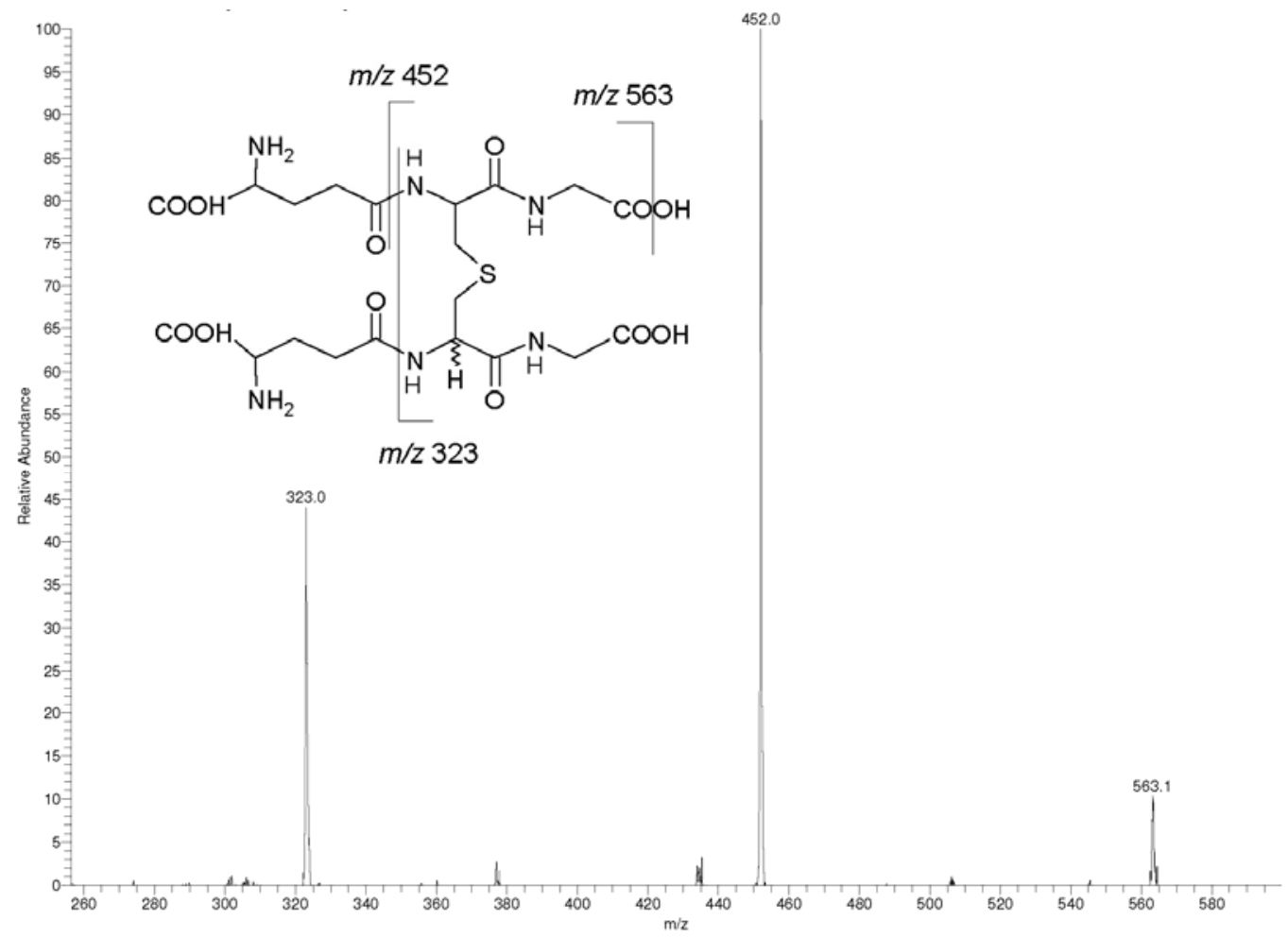

Figure 3.3.2 MS/MS Fragmentation Pattern of GSG

\subsubsection{EdAG-Glutathione conjugation catalyzed by GSTs}

To determine if GSG formation was catalyzed by GSTs, the same solution was prepared with equimolar amounts of reduced GSH and EdAG along with $0.2 \mathrm{mg}$ of human liver cytosol, which contained all soluble forms of GSTs. Aliquots were taken immediately, and then every hour until GSG formation leveled off after 3 hours. Figure 3.3.3 demonstrates the presence of EdAG and GSH at 5 minutes with minor nonenzymatic GSG formation having occurred. The yaxes are fixed to the relative abundance of the most intense signal of the three ions. 


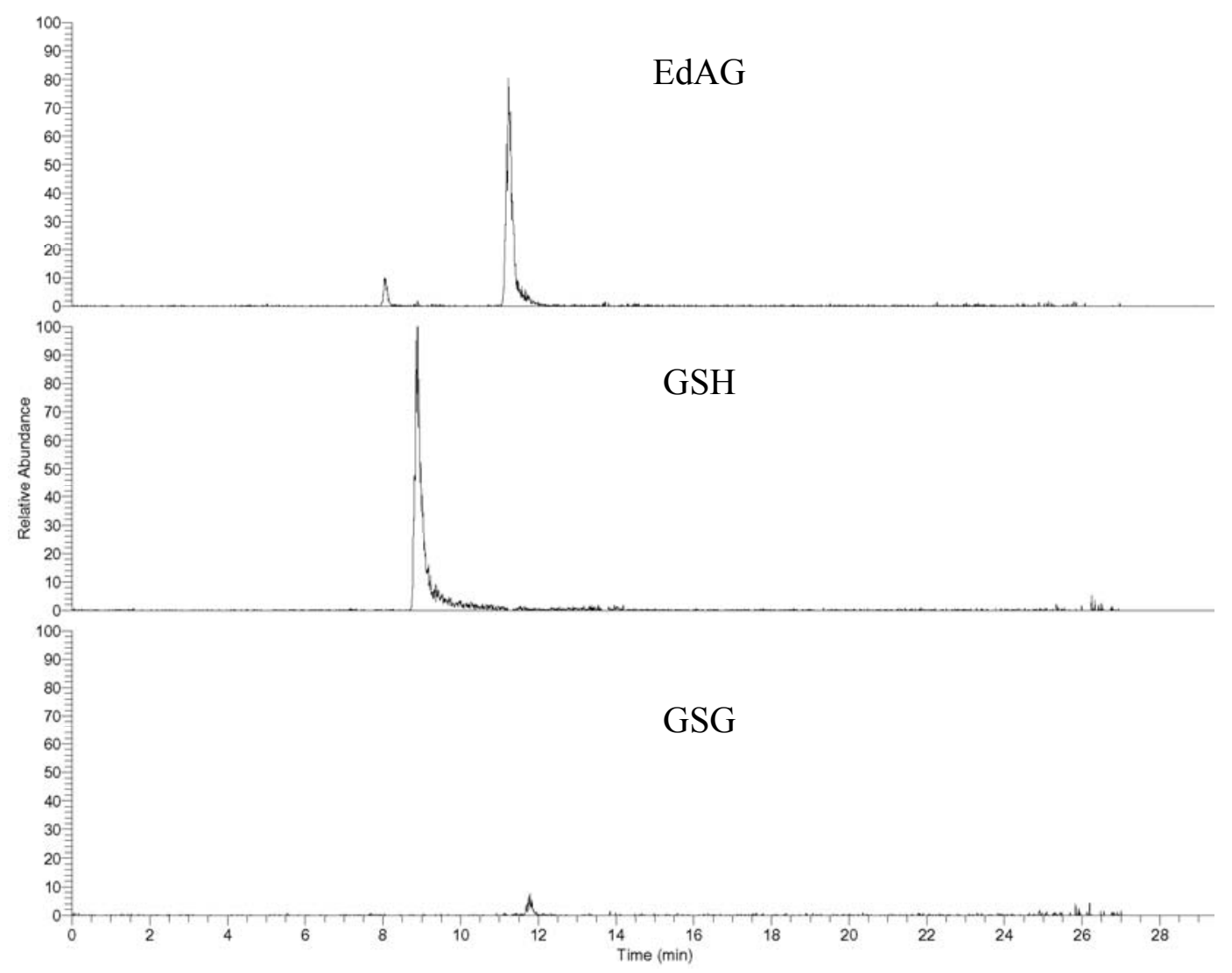

Figure 3.3.3 Nonenzymatic GSG formation after 5 minutes

Figure 3.3.4 demonstrates the changes in the relative abundance of the three ions after 180 minutes. GSG is now the most abundant ion present of the three after 3 hours. GSG formation leveled off after 3 hours due to the depletion of reduced GSH, where most was involved in GSG formation and the rest oxidized to form GSSG (data not shown). 


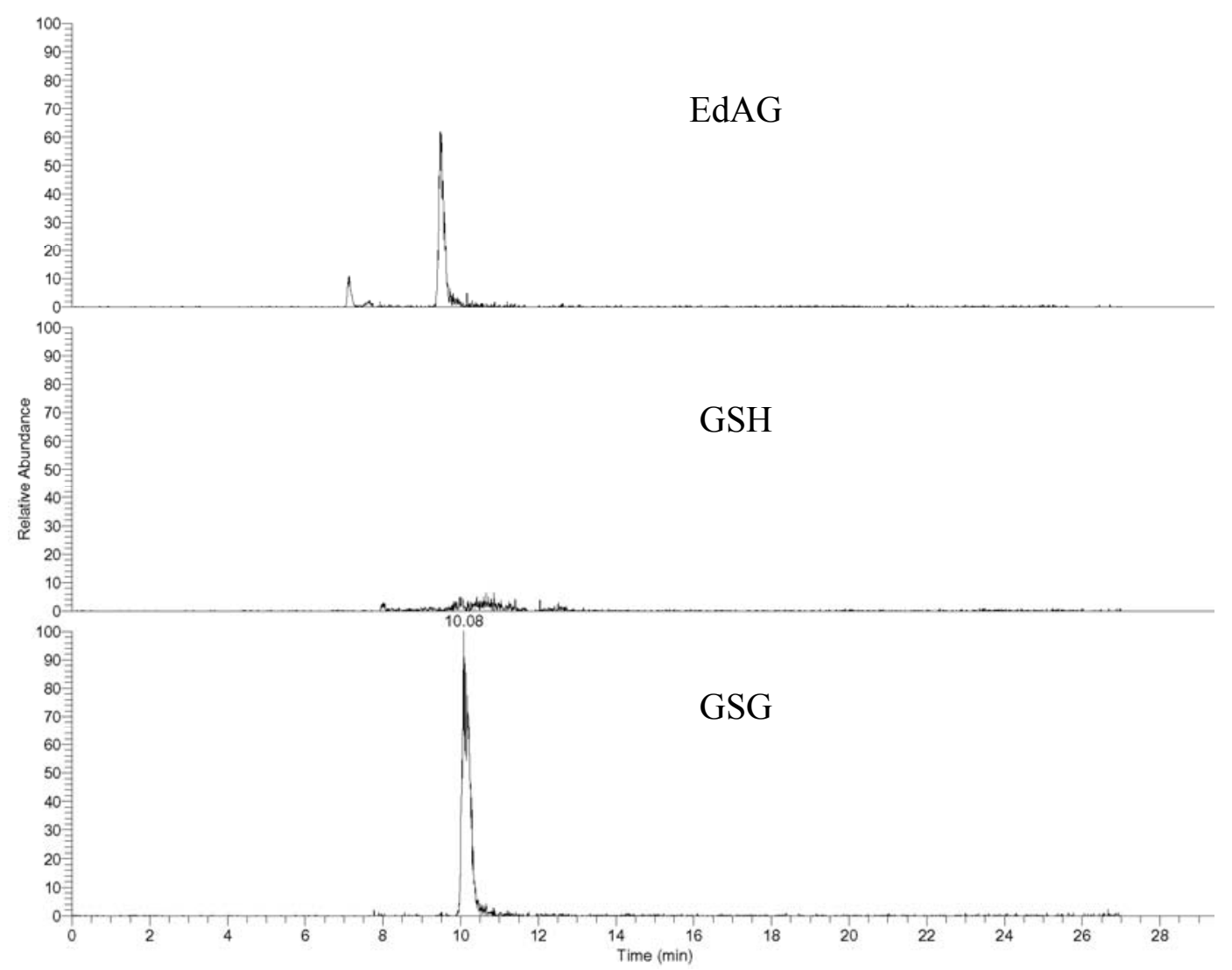

Figure 3.3.4 Nonenzymatic GSG formation after 180 minutes

In the presence of human liver cytosol, containing soluble isoforms of GST including the isoform most responsible for busulfan metabolism to EdAG (GSTA1-1), GSG formation was not increased, indicating that EdAG is not a substrate for GSTs as hypothesized. Figure 3.3.5 demonstrates the formation of GSG in the presence of GSTs after 180 minutes, with relatively less GSG formed than in the absence of GSTs after 3 hours. 


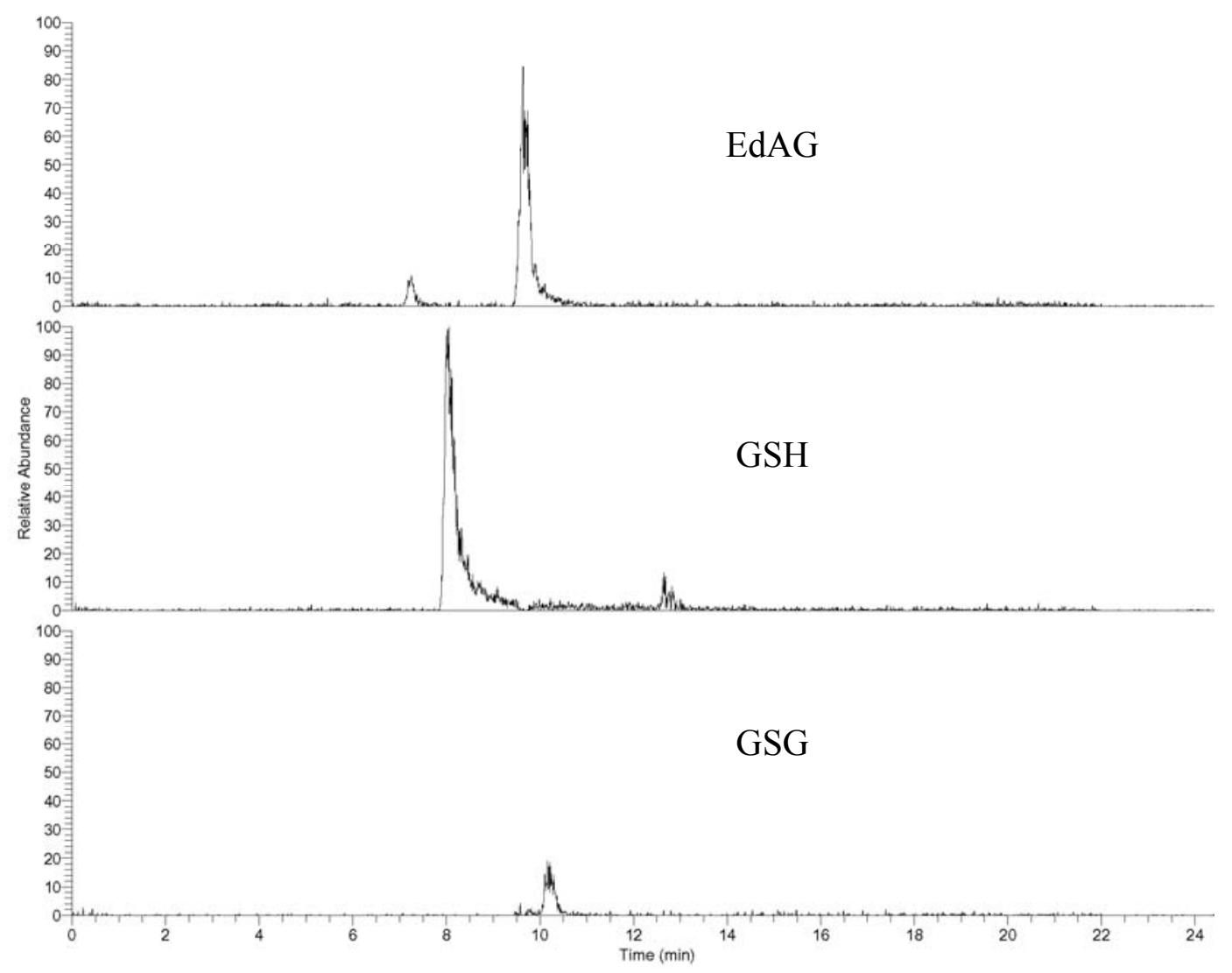

Figure 3.3.5 GSG formation in presence of GSTs after 180 minutes

\subsubsection{Reactivity of EdAG with Insulin}

The reactivity of EdAG toward thiols was extended to cysteine residues within proteins. Insulin is a relatively small protein containing 6 cysteine residues, with 4 on the A-chain and 2 on the B-chain. Within the A-chain, 2 cysteines are involved in an intra-chain disulfide bond and the other 2 cysteines are each involved in separate inter-chain disulfide bonds with the 2 cysteines on the B-chain (Figure 3.3.6). 


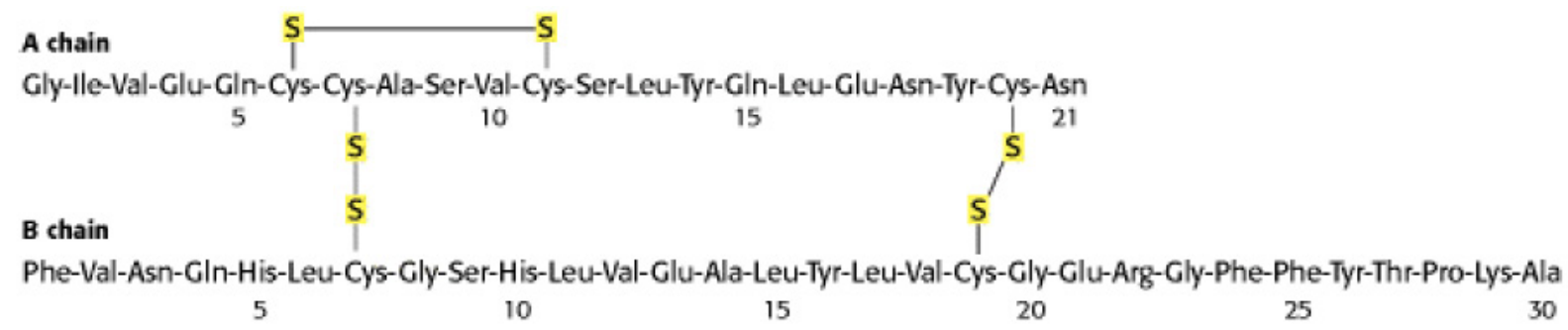

Figure 3.3.6 Bovine Insulin

Electrospray ionization (ESI) and matrix-assisted laser desorption ionization-time of flight (MALDI-TOF) mass spectrometry were both used to study both the multiply-charged ions and the singly-charged protein molecular ion, respectively. MALDI mass spectrometry utilizes a pulsed laser to softly ionize (not fragment) the analyte, while offering a higher mass range up to 8000 Da. Bovine insulin has a molecular weight of $5734 \mathrm{Da}$, which provides molecular ions (and their charge states) of m/z $1147(+5), \mathrm{m} / \mathrm{z} 1434(+4)$, and m/z $1911(+3)$ in ESI-MS (Figure 3.3.7). Intact insulin provides one molecular ion at $\mathrm{m} / \mathrm{z} 5734$ when analyzed by MALDI-TOF (data not shown). 


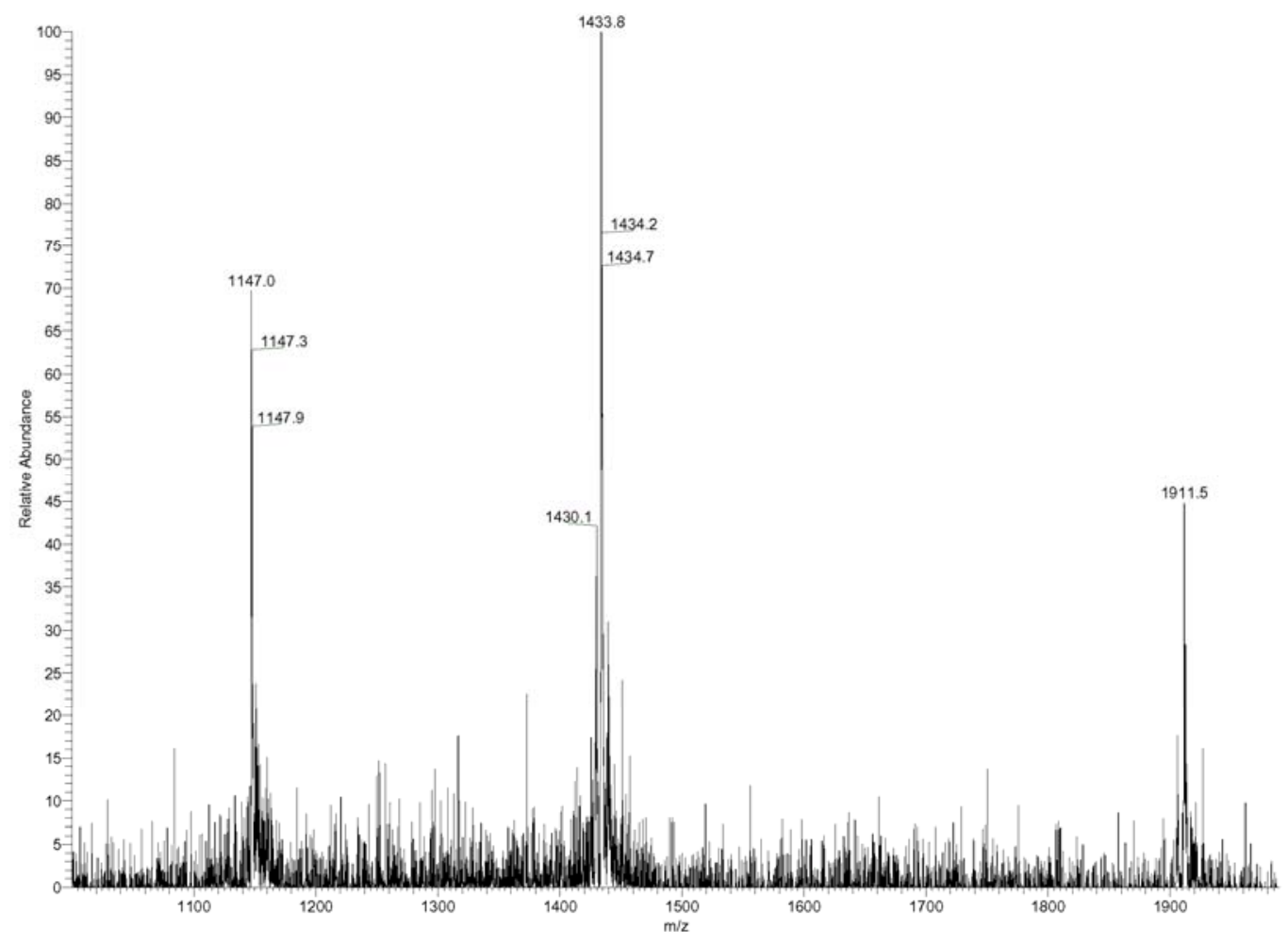

Figure 3.3.7 ESI mass spectrum of intact bovine insulin

In order for the cysteine thiols to be reactive, the two inter-chain disulfide bonds must be reduced by the reducing agent TCEP. Figure 3.3.8 depicts the ESI mass spectrum of the B-chain by the ions at $m / z 850(+4), m / z 1134(+3)$, and $m / z 1700(+2)$, along with TCEP at $m / z 251$. The MALDI mass spectrum provides a single molecular ion for the B-chain at $\mathrm{m} / \mathrm{z} 3400$ with some nonreduced, intact bovine insulin at $\mathrm{m} / \mathrm{z} 5735$ (Figure 3.3.9). 


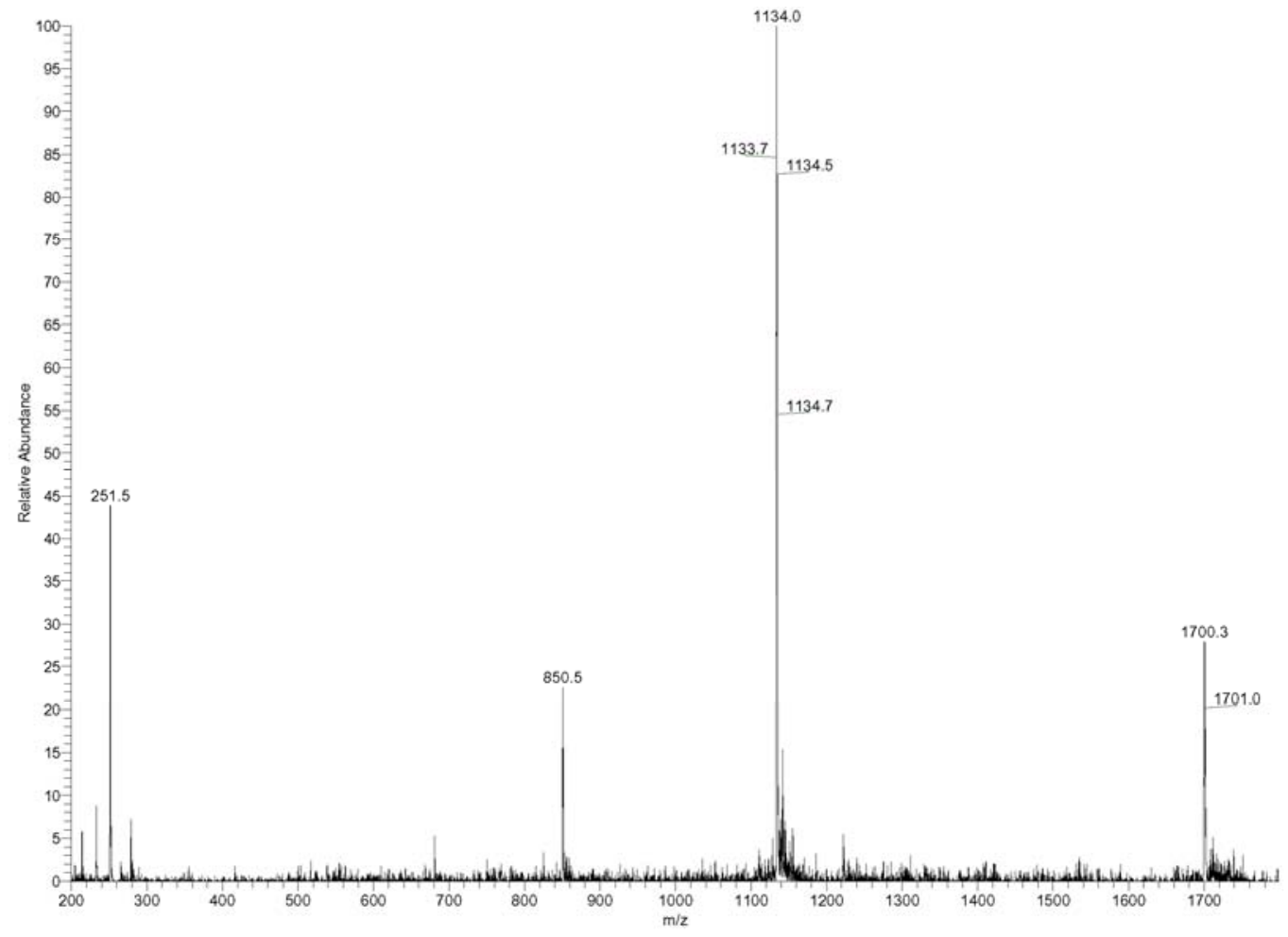

Figure 3.3.8 ESI mass spectrum of bovine insulin B-chain 


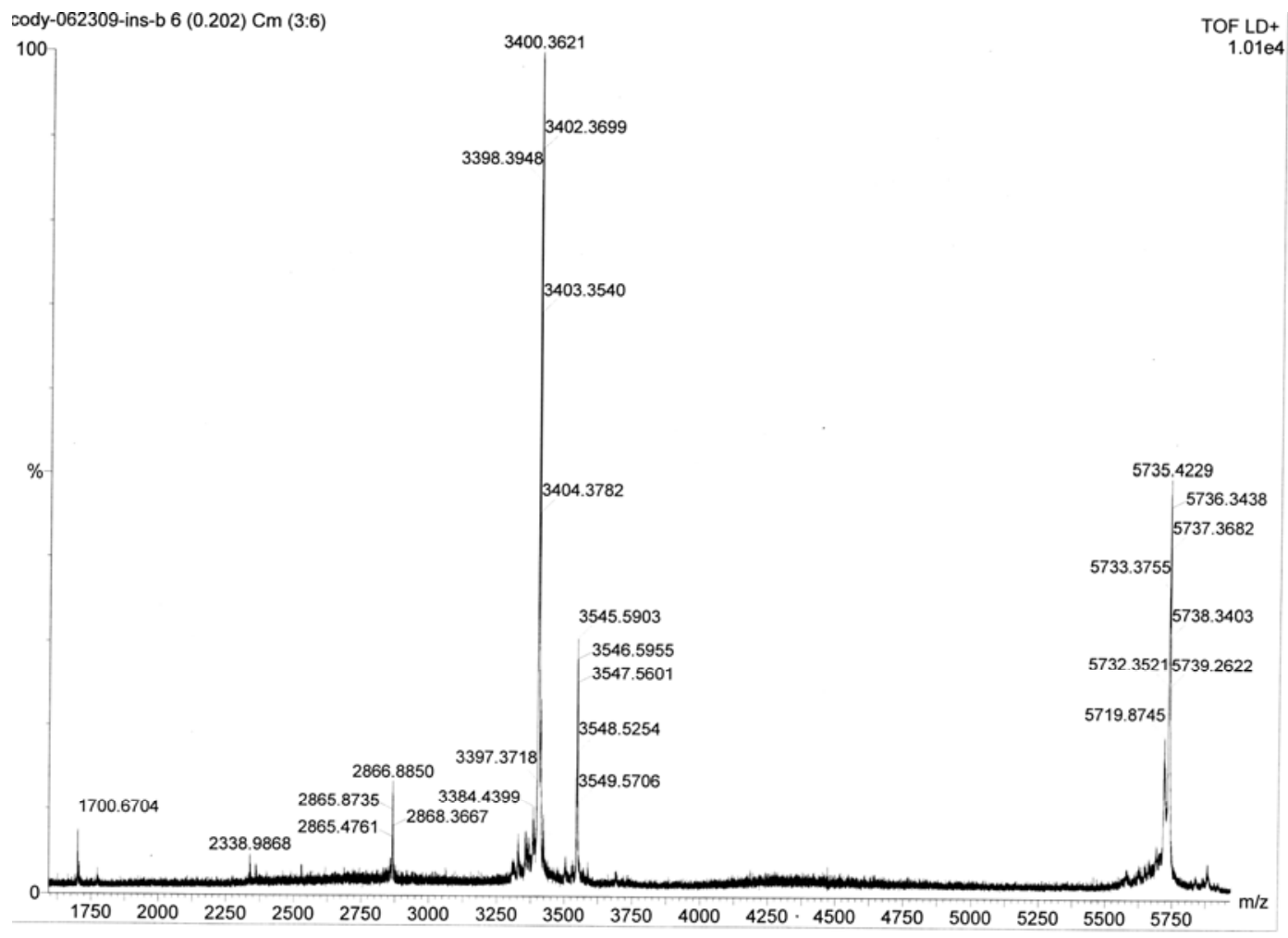

Figure 3.3.9 MALDI mass spectrum of bovine insulin B-chain

To ensure the B-chain cysteine residues are reactive towards Michael acceptors, NEM was incubated with B-chain. The ESI mass spectrum provides molecular ions at $\mathrm{m} / \mathrm{z} 913(+4)$, $\mathrm{m} / \mathrm{z} 1217(+3)$, and $\mathrm{m} / \mathrm{z} 1825(+2)$, which corresponded to the B-chain conjugated to two molecules of NEM, along with a TCEP-NEM adduct at $\mathrm{m} / \mathrm{z} 376$ (Figure 3.3.10). The singlycharged molecular ion of the B-chain $+2 \mathrm{NEM}$ adduct was demonstrated to be $\mathrm{m} / \mathrm{z} 3650$ by MALDI-TOF (Figure 3.3.11). 


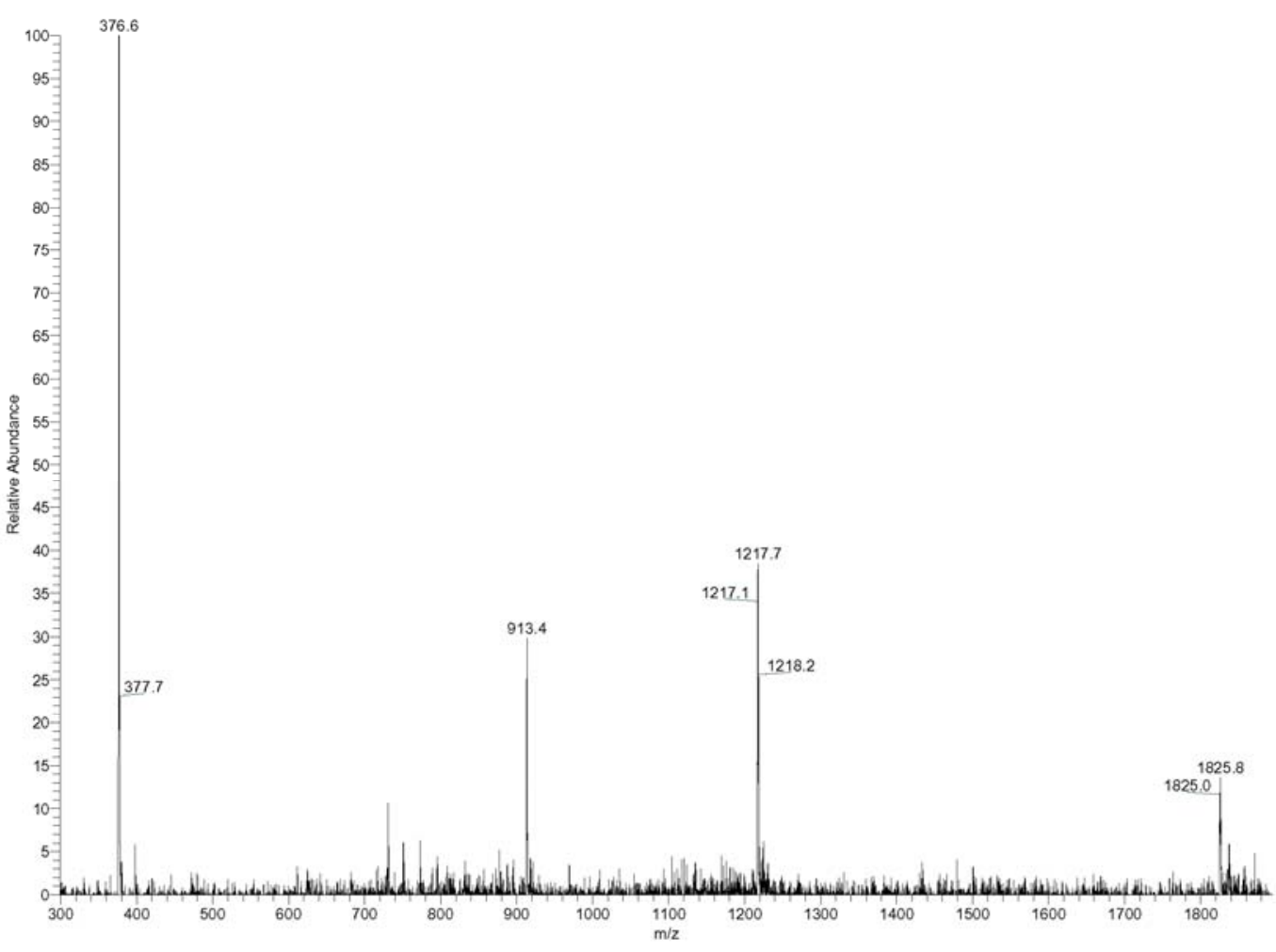

Figure 3.3.10 ESI mass spectrum of the B-chain+2NEM adduct 


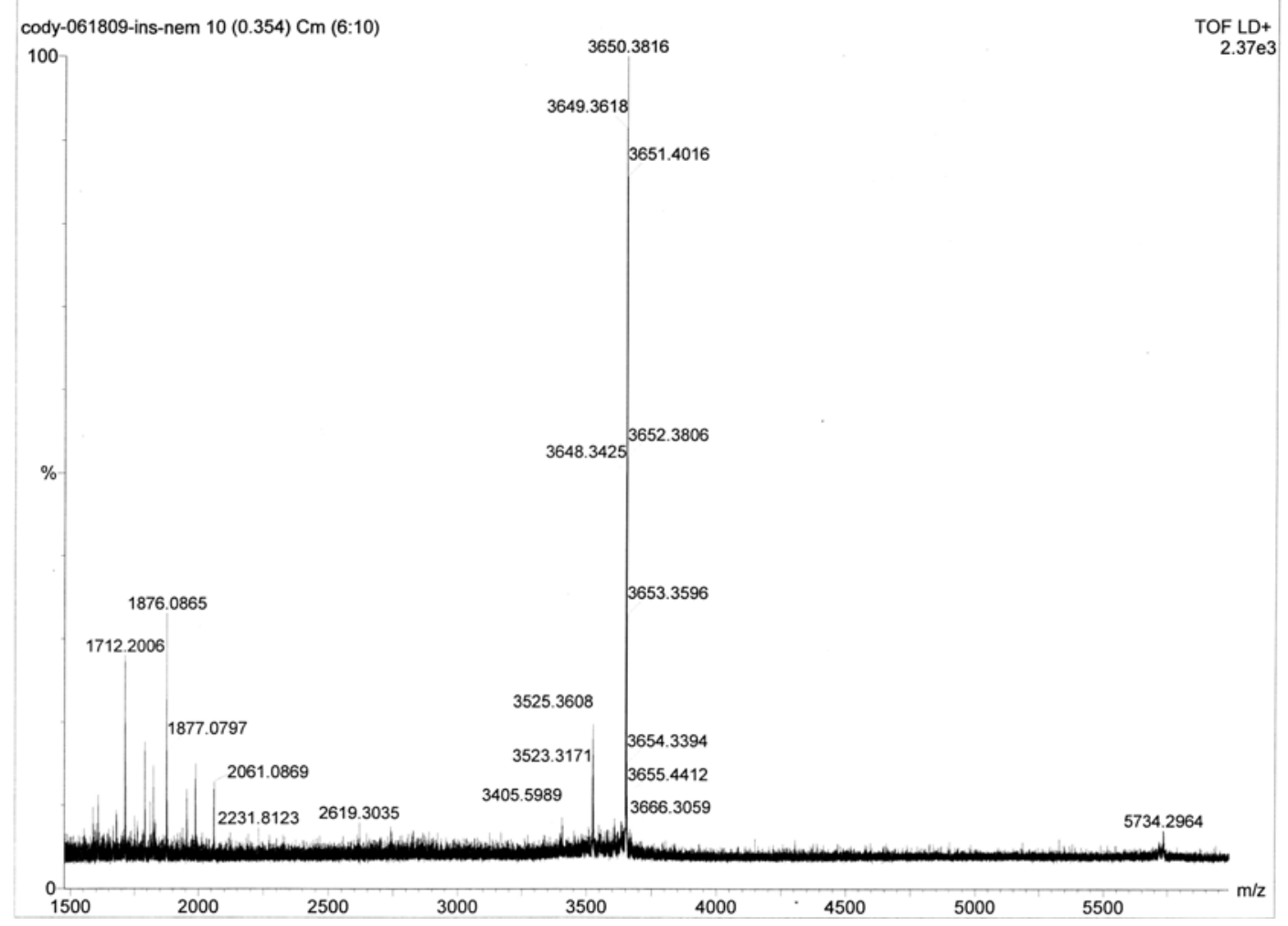

Figure 3.3.11 MALDI mass spectrum of the B-chain+2NEM adduct

Finally, EdAG was incubated with unreacted, reduced bovine insulin B-chain in order to demonstrate that the electrophilic EdAG can react with thiols of cysteine residues on proteins. The MALDI mass spectrum depicts a singly-charged molecular ion at $\mathrm{m} / \mathrm{z} 3946$ corresponding to the B-chain conjugated to two molecules of EdAG; however most of the B-chain remained unreacted at $\mathrm{m} / \mathrm{z} 3400$ (Figure 3.3.12). The multiply-charged molecular ions from ESI were not clearly recognized (data not shown). 


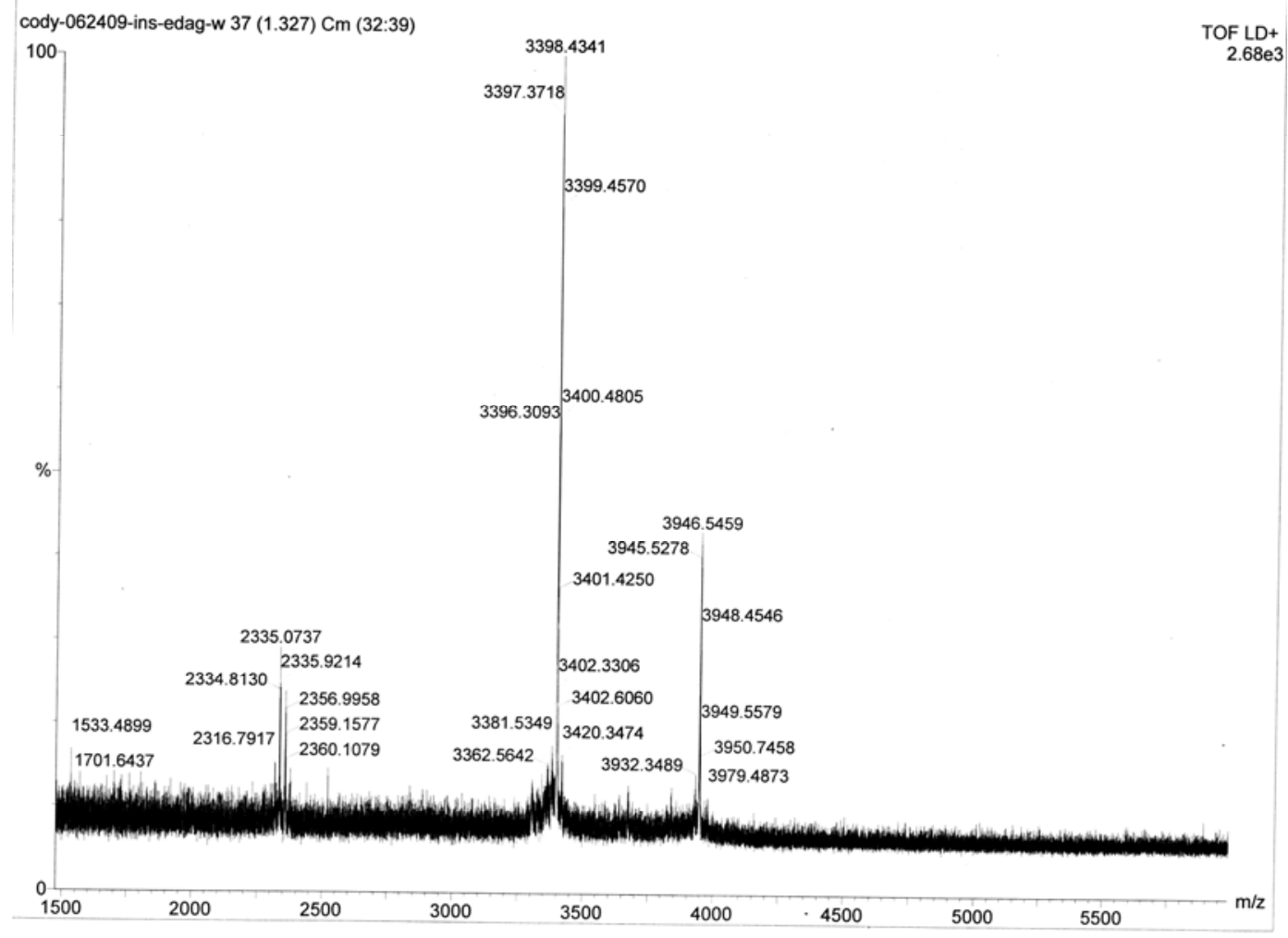

Figure 3.3.12 MALDI mass spectrum of the B-chain+2EdAG adduct

\subsubsection{Reactivity of EdAG with Albumin}

The reactivity of EdAG with a cysteine-rich and physiologically-relevant protein,

albumin, was evaluated. Human serum albumin is one of the major plasma proteins responsible for drug binding that is a marker of oxidative stress (33). Bovine serum albumin (BSA) was used as an albumin model for reactivity toward EdAG. Several attempts to alkylate the lone free cysteine at residue 34 of BSA with EdAG failed under a variety of reaction conditions, possibly due to disulfide formation between the free thiols of two BSA molecules. To activate cysteine thiols toward electrophiles, the thiol reducing agent TCEP was added to reduce all 35 cysteine residues prior to incubation with excess EdAG or NEM (as positive control). Table 3.1 provides 
the relevant trypsin-digested BSA peptides containing cysteine residues that were alkylated by EdAG.

\begin{tabular}{|l|l|l|l|l|}
\hline Residues & Sequence & Unmodified & NEM-Modified & EdAG-Modified \\
\hline $581-587$ & CCAADDK & 725.26 & 975.26 & 1271.26 \\
\hline $499-507$ & CCTESLVNR & 1024.46 & 1274.46 & 1570.46 \\
\hline $460-468$ & CCTKPESER & 1052.45 & 1302.45 & 1598.45 \\
\hline $286-297$ & YICDNQDTISSK & 1386.62 & 1511.62 & 1659.62 \\
\hline $106-117$ & ETYGDMADCCEK & 1364.48 & 1614.48 & 1910.48 \\
\hline $375-386$ & EYEATLEECCAK & 1388.57 & 1638.57 & 1934.57 \\
\hline
\end{tabular}

Table 3.1 BSA peptides from trypsin digestion

Due to the abundance of modified peptides, only the most clearly recognizable EdAG-modified peptides were presented to demonstrate the reactivity toward cysteine residues of albumin.

Figure 3.3.13 depicts the MALDI mass spectra of mass range $m / z$ 1240-1300, where (A) is NEM-alkylated BSA and (B) is EdAG-alkylated BSA. Both spectra contain signals at $\mathrm{m} / \mathrm{z}$ 1249 and $\mathrm{m} / \mathrm{z}$ 1283, which correspond to peptides that do not contain cysteine residues, and thus are not modified in either incubation. Figure 3.3.13 A contains a signal at $\mathrm{m} / \mathrm{z} 1274$, which corresponds to a 9-residue peptide from positions 499-507, that shows NEM-modified cysteines that are unique to the NEM-alkylated BSA (also see Table 3.1). Figure 3.3.13 B contains a signal at $\mathrm{m} / \mathrm{z}$ 1271, which corresponds to a 7-residue peptide from positions 581-587. This peptide is consistent with two EdAG-modified cysteines. 


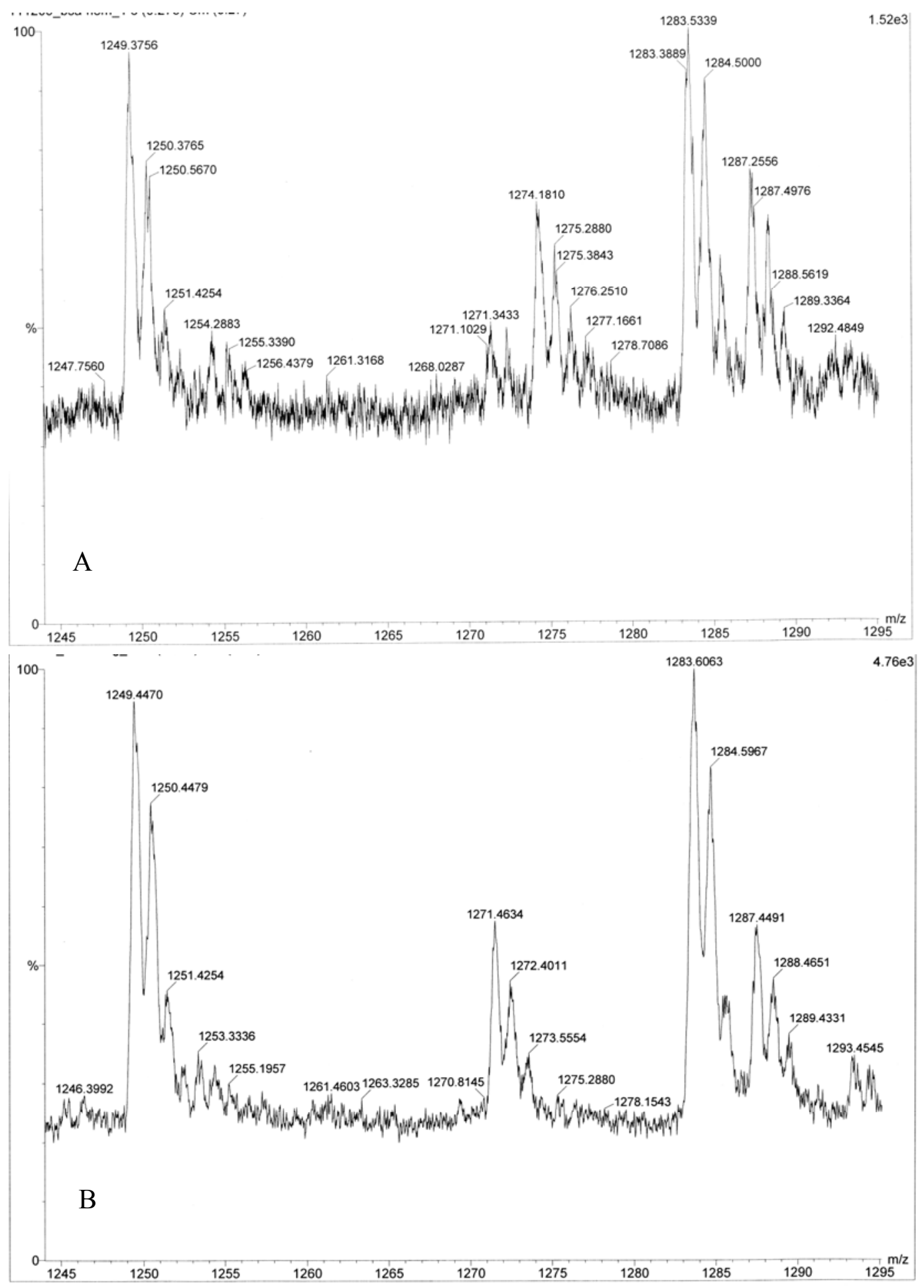

Figure 3.3.13 MALDI mass spectra of mass range $\mathrm{m} / \mathrm{z}$ 1240-1300 
Figure 3.3.14 depicts the MALDI mass range from $m / z$ 1585-1675, where (A) is the NEM-alkylated BSA and (B) is EdAG-alkylated BSA. Both spectra contain unmodified peptides with signals at $\mathrm{m} / \mathrm{z} 1590, \mathrm{~m} / \mathrm{z} 1605$, and $\mathrm{m} / \mathrm{z}$ 1640. Figure 3.3.14 A contains a unique 12-residue peptide at $\mathrm{m} / \mathrm{z} 1639$ that contains two NEM-modified cysteines corresponding to residues 375-386 (see Table 3.1). Figure 3.3.14 B contains two peptides unique to the EdAGalkylated BSA. The first is a 9-residue peptide from positions 460-468 with two EdAG-modified cysteines, corresponding to $\mathrm{m} / \mathrm{z} 1598$. The second is a 12-residue peptide from positions 286 297 with one EdAG-modified cysteine, corresponding to $\mathrm{m} / \mathrm{z} 1661$, although the expected mass was 1659 amu. This 2 mass units difference was accepted because it was unique to the EdAGalkylated BSA solution and did not correspond to any unmodified peptides. It is not known if the 2 Da difference represents a dehydrogenation reaction.

Figure 3.3.15 depicts the MALDI mass range from $m / z$ 1880-1960, where (A) is the NEM-alkylated BSA and (B) is EdAG-alkylated BSA. Both spectra contain unmodified peptides at $\mathrm{m} / \mathrm{z} 1889, \mathrm{~m} / \mathrm{z} 1927$, and $\mathrm{m} / \mathrm{z}$ 1949. Figure 3.3.15 A contains a unique 16-residue peptide from positions 508-523 with one NEM-modified cysteine corresponding to $\mathrm{m} / \mathrm{z} 1948$. Figure 3.3.15 B contains two modified peptides that are unique to EdAG-alkylated BSA. The first is a 12-residue peptide from positions 106-117 with two EdAG-modified cysteines, corresponding to $\mathrm{m} / \mathrm{z} 1911$. The second unique peptide is a 12-residue peptide from positions 375-386 with two EdAG-modified cysteines, corresponding to m/z 1934.

The EdAG-modified peptides of trypsin-digested BSA, as well as bovine insulin, are evidence of the formation of lanthionine bridges in proteins in vitro. 

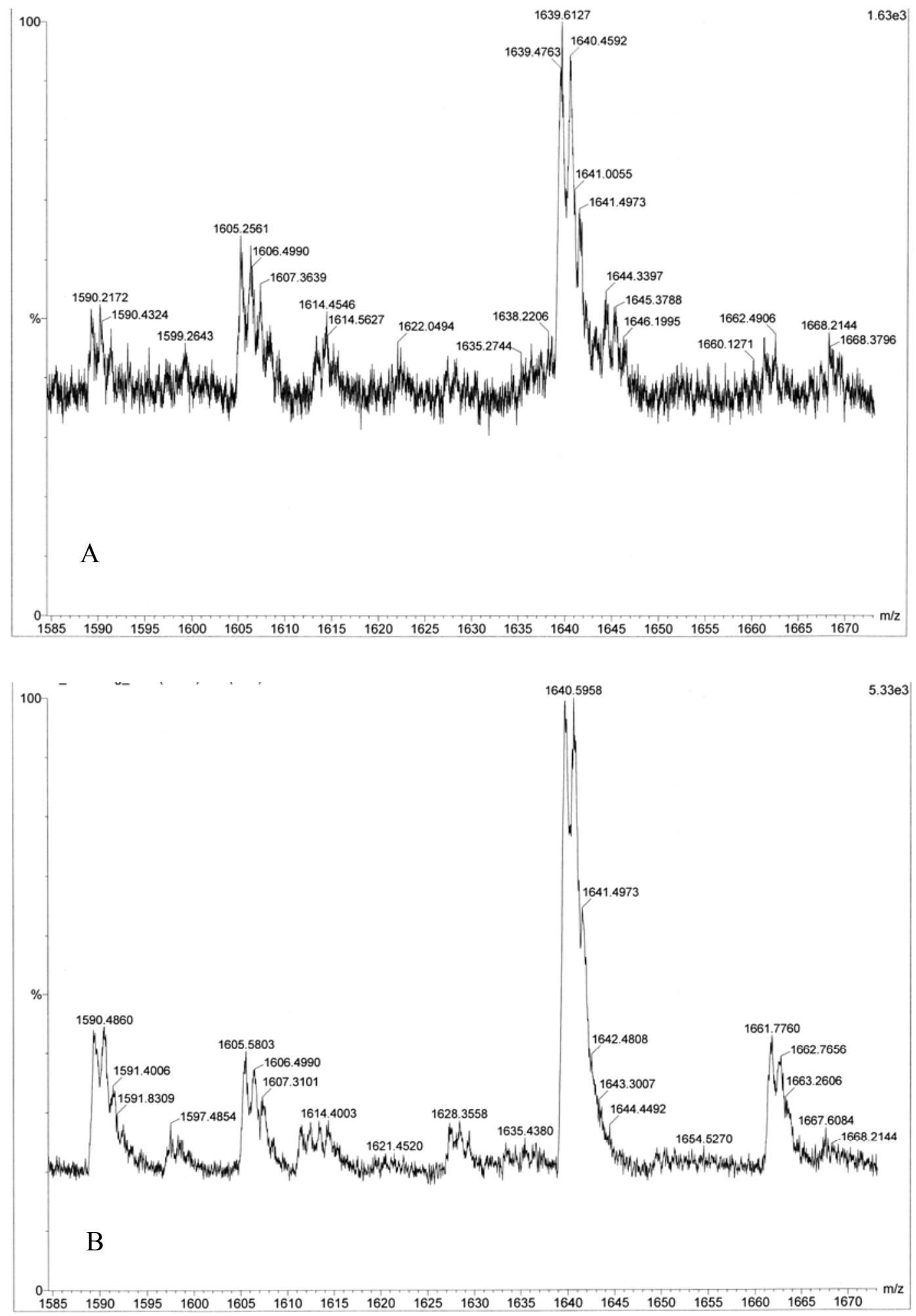

Figure 3.3.14 MALDI mass spectra of mass range m/z 1585-1675 

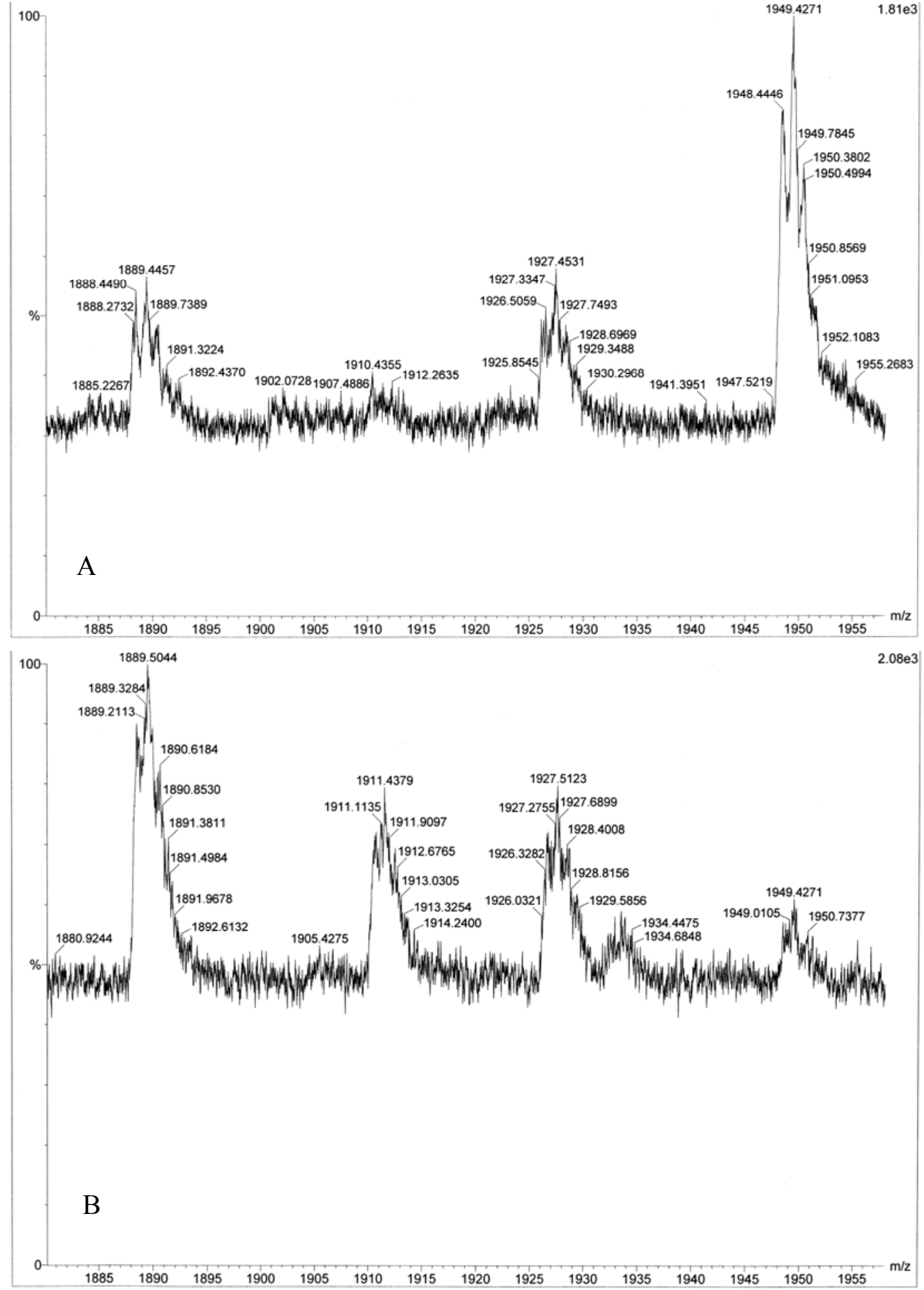

Figure 3.3.15 MALDI mass spectra of mass range m/z 1880-1960 


\section{DISCUSSION}

\section{4}

Dehydroalanines occur frequently in nature, such as in albumin, human lens proteins, and lantibiotics, as well as chemically during food processing. These dehydroalanines have been shown to be reactive with cellular nucleophiles, most notably GSH, which subsequently forms a non-reducible lanthionine bond.

The reactivity of dehydroalanines was analogous to the electrophilic dehydroalanine analog of glutathione, EdAG, which is a metabolite of the anticancer agent busulfan. Both the amino acid L-cysteine, which contains a nucleophilic side chain thiol, and the most abundant form of cellular thiols, the tripeptide glutathione, were reacted with EdAG to determine the extent of conjugation that occurs nonenzymatically. When equimolar amounts of EdAG and either L-cysteine or reduced glutathione were incubated in solution under physiological conditions of $\mathrm{pH} 7.4$ and $37^{\circ} \mathrm{C}$, conjugation of the thiol to EdAG was detected by mass spectrometry. Tandem mass spectrometry (MS/MS) was used, employing CID to confirm the structure of each adduct to ensure the correct conjugate was formed. These experiments suggest that the dehydroalanine moiety in EdAG is sufficiently reactive towards cellular thiols when present as the individual amino acid cysteine or the tripeptide GSH. The non-reducible lanthionine bond was formed by nucleophilic addition of a cysteine, either L-cysteine or GSH, to a dehydroalanine residue in a tripeptide. Thus, EdAG could serve as a model peptide-containing dehydroalanine to further study lanthionine formation in vivo.

Glutathione is a substrate for all GSTs and due to the high intracellular levels of GSH, most cytosolic GSTs have GSH constantly bound in the hydrophilic glutathione binding site (G- 
site). Hydrophobic substrates, such as busulfan, bind to the hydrophobic binding site (H-site), which positions the substrate near the reactive thiol of GSH (actually the thiyl GS-) to induce conjugation. EdAG was shown to bind GSTA1-1 on a GSH-agarose gel (34), but that does not necessarily imply that EdAG is a ligand of either the G- or H-site. EdAG and GSH were incubated in the presence of GSTs to determine if EdAG could bind the H-site and position itself in close proximity to the reactive thiol of GSH in the G-site of GSTs such that GSG formation is catalyzed by GSTs. After 3 hours, the reaction was complete and there was no significant increase in GSG formation in the presence of GSTs relative to the nonenzymatic equimolar incubation of EdAG and GSH. This outcome was expected because EdAG is a hydrophilic molecule and probably doesn't bind well or at all in the H-site, thus catalysis for GSG formation is less likely.

The glutathionylation of proteins is a common post-translational modification (PTM), which normally occurs when GSH attacks a cysteine or a dehydroalanine residue on a protein. The dehydroalanine residue was originally either a cysteine or a serine that lost the heteroatom functional group as a PTM. It was desired here to determine if EdAG could react with cysteine residues on proteins to essentially glutathionylate the proteins, albeit in the reverse order with the cysteine residue attacking the dehydroalanine of EdAG. Insulin was used as a model protein that contains two reactive cysteine residues when reduced to its B-chain. Both electrospray ionization (ESI), which produces multiply-charged molecular ions, and matrix-assisted laser desorption ionization (MALDI), which produces singly-charged molecular ions, were used to study the insulin conjugates with the Michael acceptors N-ethyl maleimide (NEM) and EdAG. NEM is a well-known Michael acceptor and was used as a model electrophile to demonstrate the ability of Michael acceptors to be conjugated by the reactive cysteine thiols of reduced bovine 
insulin B-chain. NEM proved to be an excellent model for Michael acceptors, with mostly all the B-chain reacting with NEM. Because there are two reactive cysteine residues on each Bchain, there are two NEM molecules added to the B-chain, counting for an additional molecular weight of $250 \mathrm{amu}$. When incubated in the same molar ratio to B-chain as in the NEM reaction, EdAG also reacted with B-chain. Two EdAG molecules were conjugated per B-chain, accounted for by the additional molecular weight of 546 amu. However, there was mostly unreacted B-chain present in the MALDI mass spectrum (Figure 3.3.11), and the B-chain-EdAG adduct was a minor product. This suggests EdAG is a reactive Michael acceptor, but not as reactive as NEM. The evidence that EdAG is a relatively weak Michael acceptor is supported by Li et al that suggested electron donation by the enamine functionality weakens the electrophilicity of dehydroalanines in comparison with the electrophilicity of more classical Michael acceptor compounds (35).

In conclusion, the electrophilic dehydroalanine analog of glutathione (EdAG) was shown to be reactive towards the cellular nucleophiles L-cysteine, GSH, and cysteine residues on bovine insulin B-chain and bovine serum albumin. This reactivity demonstrated the potential of EdAG to trap intracellular GSH with a non-reducible thioether bond that could further deplete GSH levels when the anticancer agent busulfan is administered. Also, EdAG has the potential to bind cellular proteins and form non-reducible lanthionines that could alter their function and disrupt cellular processes. One protein of particular interest that EdAG could react with is the enzyme responsible for its formation, GSTA1-1. EdAG was shown to bind GSTA1-1 from studies by Younis et al (34), however it was suggested that EdAG is not a substrate of the H-site of GSTs, due to the lack of GST-catalyzed GSG formation. EdAG could however be a substrate of the G-site due to its structural similarities to GSH, with the one obvious exception of the lack 
of the thiol group. In the next chapter, it was desired to determine the extent of EdAG binding in the G-site and if EdAG could act as a competitive inhibitor of GSTs, particularly GSTA1-1 because it is the most abundant cytosolic GST isoform in the liver and is also the predominant isoform responsible for EdAG formation from busulfan. 


\section{REFERENCES}

(1) Boyland, E., and Chasseaud, L. F. (1967) Enzyme-catalysed conjugations of glutathione with unsaturated compounds. Biochem. J. 104(1), 95-102.

(2) Lopez-Mirabal, H. R., and Winther, J. R. (2008) Redox characteristics of the eukaryotic cytosol. Biochim. Biophys. Acta 1783(4), 629-640.

(3) Mannervik, B., Awasthi, Y. C., Board, P. G., Hayes, J. D., Di, I. C., Ketterer, B., Listowsky, I., Morgenstern, R., Muramatsu, M., Pearson, W. R., and . (1992) Nomenclature for human glutathione transferases. Biochem. J. 282 ( Pt 1), 305306.

(4) Friedman, M., Finley, J. W., and Yeh, L. S. (1977) Reactions of proteins with dehydroalanines. Adv. Exp. Med. Biol. 86B, 213-224.

(5) Kim, J. S., and Kim, H. J. (2001) Matrix-assisted laser desorption/ionization time-offlight mass spectrometric observation of a peptide triplet induced by thermal cleavage of cystine. Rapid Commun. Mass Spectrom. 15(23), 2296-2300.

(6) Nakanishi, T., Sato, T., Sakoda, S., Yoshioka, M., and Shimizu, A. (2004) Modification of cysteine residue in transthyretin and a synthetic peptide: analyses by electrospray ionization mass spectrometry. Biochim. Biophys. Acta 1698(1), 4553.

(7) Thakur, S. S., and Balaram, P. (2008) Fragmentation of peptide disulfides under conditions of negative ion mass spectrometry: studies of oxidized glutathione and contryphan. J. Am. Soc. Mass Spectrom. 19(3), 358-366. 
(8) Bar-Or, R., Rael, L. T., and Bar-Or, D. (2008) Dehydroalanine derived from cysteine is a common post-translational modification in human serum albumin. Rapid Commun. Mass Spectrom. 22(5), 711-716.

(9) Grune, T., Reinheckel, T., and Davies, K. J. (1997) Degradation of oxidized proteins in mammalian cells. FASEB J. 11(7), 526-534.

(10) Linetsky, M., Hill, J. M., LeGrand, R. D., and Hu, F. (2004) Dehydroalanine crosslinks in human lens. Exp. Eye Res. 79(4), 499-512.

(11) Srivastava, O. P., Kirk, M. C., and Srivastava, K. (2004) Characterization of covalent multimers of crystallins in aging human lenses. J. Biol. Chem. 279(12), 1090110909.

(12) Finley, J. W., and Friedman, M. (1977) New amino acid derivatives formed by alkaline treatment of proteins. Adv. Exp. Med. Biol. 86B, 123-130.

(13) Cloos, P. A., and Jensen, A. L. (2000) Age-related de-phosphorylation of proteins in dentin: a biological tool for assessment of protein age. Biogerontology. 1(4), 341356.

(14) Kamei, A. (1993) Glutathione levels of the human crystalline lens in aging and its antioxidant effect against the oxidation of lens proteins. Biol. Pharm. Bull. 16(9), $870-875$.

(15) Linetsky, M., and LeGrand, R. D. (2005) Glutathionylation of lens proteins through the formation of thioether bond. Mol. Cell Biochem. 272(1-2), 133-144.

(16) Bodanszky, M., Scozzie, J. A., and Muramatsu, I. (1970) Dehydroalanine residues in thiostrepton. J. Antibiot. (Tokyo) 23(1), 9-12. 
(17) Consevage, M. W., and Phillips, A. T. (1985) Presence and quantity of dehydroalanine in histidine ammonia-lyase from Pseudomonas putida. Biochemistry 24(2), 301308.

(18) Corpet, D. E., Tache, S., Archer, M. C., and Bruce, W. R. (2008) Dehydroalanine and lysinoalanine in thermolyzed casein do not promote colon cancer in the rat. Food Chem. Toxicol. 46(9), 3037-3042.

(19) Jensen, P. H., Laursen, I., Matthiesen, F., and Hojrup, P. (2007) Posttranslational modifications in human plasma MBL and human recombinant MBL. Biochim. Biophys. Acta 1774(3), 335-344.

(20) Gavaret, J. M., Nunez, J., and Cahnmann, H. J. (1980) Formation of dehydroalanine residues during thyroid hormone synthesis in thyroglobulin. J. Biol. Chem. 255(11), 5281-5285.

(21) Gupta, A., and Chauhan, V. S. (1990) Synthetic and conformational studies on dehydroalanine-containing model peptides. Biopolymers 30(3-4), 395-403.

(22) Hallinan, E. A. (1991) Formation of a dehydroalanyl residue from S-benzylcysteine upon HF cleavage of a [Sar1, Cys8]-angiotensin II peptide resin. Int. J. Pept. Protein Res. 38(6), 601-602.

(23) Hodgins, D. S. (1971) Yeast phenylalanine ammonia-lyase. Purification, properties, and the identification of catalytically essential dehydroalanine. J. Biol. Chem. 246(9), 2977-2985.

(24) Levengood, M. R., and van der Donk, W. A. (2006) Dehydroalanine-containing peptides: preparation from phenylselenocysteine and utility in convergent ligation strategies. Nat. Protoc. 1(6), 3001-3010. 
(25) Lombardi, A., D'Agostino, B., Nastri, F., D'Andrea, L. D., Filippelli, A., Falciani, M., Rossi, F., and Pavone, V. (1998) A novel super-potent neurokinin A receptor antagonist containing dehydroalanine. Bioorg. Med. Chem. Lett. 8(10), 11531156.

(26) Murkin, A. S., and Tanner, M. E. (2002) Dehydroalanine-based inhibition of a peptide epimerase from spider venom. J. Org. Chem. 67(24), 8389-8394.

(27) Naidu, B. N., Sorenson, M. E., Connolly, T. P., and Ueda, Y. (2003) Michael addition of amines and thiols to dehydroalanine amides: a remarkable rate acceleration in water. J. Org. Chem. 68(26), 10098-10102.

(28) Schuster, B., and Retey, J. (1995) The mechanism of action of phenylalanine ammonialyase: the role of prosthetic dehydroalanine. Proc. Natl. Acad. Sci. U. S. A 92(18), 8433-8437.

(29) Srinivasan, A., Stephenson, R. W., and Olsen, R. K. (1977) Synthesis of dehydroalanine peptides from beta-chloroalanine peptide derivatives. J. Org. Chem. 42(13), 22532256.

(30) Tomkinson, B., Grehn, L., Fransson, B., and Zetterqvist, O. (1994) Use of a dehydroalanine-containing peptide as an efficient inhibitor of tripeptidyl peptidase II. Arch. Biochem. Biophys. 314(2), 276-279.

(31) Wang, J., Schiller, S. M., and Schultz, P. G. (2007) A biosynthetic route to dehydroalanine-containing proteins. Angew. Chem. Int. Ed Engl. 46(36), 68496851.

(32) Li, F., Chordia, M. D., Woodling, K. A., and Macdonald, T. L. (2007) Irreversible alkylation of human serum albumin by zileuton metabolite 2- 
acetylbenzothiophene-S-oxide: a potential model for hepatotoxicity. Chem. Res. Toxicol. 20(12), 1854-1861.

(33) Himmelfarb, J., and McMonagle, E. (2001) Albumin is the major plasma protein target of oxidant stress in uremia. Kidney Int. 60(1), 358-363.

(34) Younis, I. R., Elliott, M., Peer, C. J., Cooper, A. J., Pinto, J. T., Konat, G. W., Kraszpulski, M., Petros, W. P., and Callery, P. S. (2008) Dehydroalanine analog of glutathione: an electrophilic busulfan metabolite that binds to human glutathione S-transferase A1-1. J. Pharmacol. Exp. Ther. 327(3), 770-776.

(35) Li, G., and van der Donk, W. A. (2007) Efficient synthesis of suitably protected betadifluoroalanine and gamma-difluorothreonine from L-ascorbic acid. Org. Lett. $9(1), 41-44$. 


\title{
Chapter 4
}

\section{EdAG-Mediated Inhibition of Glutathione-S}

\author{
Transferases
}




\section{INTRODUCTION}

\subsubsection{Glutathione-S Transferases (GSTs) Superfamily of enzymes}

GSTs use reduced glutathione (GSH) to adduct electrophilic xenobiotics, such as busulfan, to aid in their metabolism and elimination from the body. There are eight major families of mammalian GSTs (alpha, kappa, mu, omega, pi, sigma, theta, and zeta) (1-5), where the key residue for GSH activation is a serine for the theta class, but is a tyrosine for all others $(6,7)$. The tyrosine residue is acidic and helps stabilize the bound GSH to allow a basic residue, suggested to be Arg15 (8), to activate the GSH by deprotonating the thiol group. The use of Arg15 in forming the thiyl group is exclusive to isoform GSTA1-1. These interactions lower the pKa of the GSH thiol group from 9.0 to a range between 6.9-6.0, allowing the formation of the thiyl conjugate $\left(\mathrm{GS}^{-}\right)$at physiological $\mathrm{pH} 7.4$ and causing a 200-300-fold rate increase in the GSH-substrate conjugation $(9,10)$. The structurally-conserved tyrosine for GST class alpha is Tyr-9, class mu is Tyr-6, and class pi is Tyr-7 (6). GSTs allow glutathione to conjugate electrophilic substrates, which in this case is busulfan. Although busulfan has been shown to be metabolized by three of the major cytosolic isoforms of GSTs (alpha, mu, and pi), GSTA1-1 is the predominant isoenzyme responsible due to its high abundance in liver (11).

\subsubsection{GST A1-1}

GSTA1-1 is a cytosolic enzyme existing as a homodimer, with each subunit containing 222 amino acids. GSTA1-1 has been shown to be the predominant isoenzyme responsible for busulfan conjugation and metabolism (11). In addition to the predominant enzyme for busulfan metabolism, GSTA1-1 is also the most abundant isoenzyme found in human liver comprising 
approximately $3 \%$ of soluble proteins in human hepatocytes $(2,12)$. Each of the two subunits in the homodimeric GSTA1-1 contain a catalytic site, consisting of a hydrophilic GSH-binding site (G-site), and a hydrophobic substrate-binding site (H-site), where the hydrophobic busulfan binds. GSTA1-1 is known to metabolize a wide variety of different hydrophobic substrates due to its substrate promiscuity (13). The topography of the H-site determines the substrate specificity among the various isoenzymes of the cytosolic GST family (14). There also exists an intersubunit solvent-accessible cleft on most GSTs. On GSTA1-1, the cleft is predominantly positively charged and has been shown to have greater affinity towards hydrophilic, multivalent negatively charged compounds (15). Among the substrates believed to bind this cleft are bilirubin $(16,17)$ and steroids $(18)$, where both have been shown to noncompetitively inhibit through a mechanism involving binding a site other that the catalytic site.

EdAG has been previously shown to bind GSTA1-1 (19), although that does not necessarily imply that EdAG is a ligand for GSTA1-1 in either of the two binding sites (G-, Hsite). Previous work in this dissertation has demonstrated that the reaction between EdAG and GSH is not catalyzed by GSTs, even though GSH is a substrate of the enzyme. This indicated that EdAG is not a substrate for the hydrophobic substrate binding site (H-site). Due to the structural similarities of EdAG and GSH, it was hypothesized that EdAG could compete with GSH for binding in the G-site and competitively inhibit GSTs, namely isoform GSTA1-1. 


\section{EXPERIMENTAL PROCEDURES}

\subsubsection{Materials}

GSH, 1-chloro-2,4-dinitrobenzene (CDNB), $\alpha$-cyano-4-hydroxycinnamic acid, and Tris buffer, pH 7.4, were purchased from Sigma-Aldrich (St. Louis, MO). All water used was deionized with a NANOpure ${ }^{\circledR}$ water purification system (Barnstead, San Jose, CA). Pooled human liver cytosol (HLC) from multiple donors, containing all cytosolic isoforms of GST (20 $\mathrm{mg} / \mathrm{mL}$ total protein concentration) was purchased from BD Gentest (Bedford, MA). Pure human recombinant GSTA1-1 $(2.5 \mathrm{mg} / \mathrm{mL})$ was purchased from US Biological (Swampscott, MA). Microcon-10 filters (Millipore Corp., Billerica, MA) with a mass cutoff of $10 \mathrm{kDa}$ was used to retain GST from solution.

\subsubsection{Instrumentation}

Ultraviolet (UV) absorbance was measured on a Beckman DU $640 \mathrm{UV} / \mathrm{Vis}$ spectrophotometer (Beckman-Coulter, Fullerton, CA). Matrix-assisted laser desorption ionization-time of flight (MALDI-TOF) mass spectrometric analyses were performed on a Micromass Reflectron MALDI-TOF, (Waters, Milford, MA).

\subsubsection{Inhibition of GSTs in Human Liver Cytosol by EdAG}

Inhibition of GST by EdAG was determined using a modified established GST activity assay (20) using CDNB, GSH, and human liver cytosol (containing most soluble GST isoenzymes) as the catalyst. CDNB (final conc $1 \mathrm{mM}$ ) in methanol (final methanol conc less than $0.5 \%$ ), and GSH (final conc $1 \mathrm{mM}$ ) in Tris buffer, $\mathrm{pH} 7.4$ were mixed and diluted with 
water to a final volume of $2 \mathrm{~mL}$ before addition of human liver cytosol (final conc $10 \mu \mathrm{g}$ protein $/ \mathrm{mL}$ ) to start the reaction. The reaction was monitored over $5 \mathrm{~min}$ for the change in absorbance at $340 \mathrm{~nm}$. CDNB concentration was held constant at $1 \mathrm{mM}$ to saturate the $\mathrm{H}$-site, while varying concentrations of GSH in the G-site to determine competitive inhibition at the Gsite with EdAG. Specific activities (or reaction velocities) were calculated as the velocity of product S-(2,4-dinitrophenyl)glutathione, or GS-DNB, formed per unit of enzyme over 5 minutes using the extinction coefficient of $\varepsilon=9.6 \mathrm{mM}^{-1} \mathrm{~cm}^{-1}(20)$ in the equation below.

$v=\frac{d A_{340 \mathrm{~nm}} / d T / 9,6 \mathrm{mM}^{-1}}{[E]}$, where $\mathrm{dA}_{340 \mathrm{~nm}} / \mathrm{dt}$ is the change in absorbance at $340 \mathrm{~nm}$ over time (5 minutes), and $[\mathrm{E}]$ is the enzyme concentration. Specific activity is expressed in $\mu \mathrm{moles} / \mathrm{mg} / \mathrm{min}$. GSH concentrations were $0.01,0.05,0.1,0.5$, and $1.0 \mathrm{mM}$. EdAG concentrations were $0,0.01$, 0.1, 0.5 and $1.0 \mathrm{mM}$. Each experiment was performed twice and average velocities were plotted by SigmaPlot 8.0 (Systat Software, Inc., Point Richmond, CA) to calculate kinetic parameters.

\subsubsection{Inhibition of human GSTA1-1 by EdAG}

Inhibition of GSTA1-1 by EdAG was determined using the same procedure as above with $\mathrm{CDNB}$, GSH, and pure recombinant human GSTA1-1 as the catalyst. CDNB (final conc 1 $\mathrm{mM}$ ) in methanol (final methanol conc less than $0.5 \%$ ), and GSH (conc ranging $0.01-1.0 \mathrm{mM}$ ) in Tris buffer, $\mathrm{pH} 7.4$ were mixed and diluted with water to a final volume of $2 \mathrm{~mL}$ before addition of pure GSTA1-1 (final conc $62.5 \mathrm{ng} / \mathrm{mL}$ ) to start the reaction. Specific activities (or reaction velocities) were calculated as the velocity of GS-DNB formed per unit of enzyme over 5 minutes 
using the extinction coefficient of $\varepsilon=9.6 \mathrm{mM}^{-1} \mathrm{~cm}^{-1}$ (20). EdAG-induced GSTA1-1 inhibition was assayed at the same EdAG concentrations of $0,0.01,0.1,0.5$ and $1.0 \mathrm{mM}$.

\subsubsection{Irreversibility of EdAG-mediated inhibition of GSTA1-1}

Pure GSTA1-1 $(1.25 \mu \mathrm{g})$ was exposed to varying amounts of EdAG for $30 \mathrm{~min}$ at room temperature to allow for potential irreversible binding to occur based on a previous study (21). The solution was then filtered through a Microcon-10 filter apparatus to remove unreacted excess EdAG and retain GSTA1-1. To condition the filters before use, a pre-wash of $100 \mu \mathrm{L}$ of $100 \mathrm{mM}$ Tris buffer, $\mathrm{pH} 7.4$ was performed with the filters centrifuged at 10,000 rpm $(13,725 \mathrm{x}$ g) for $30 \mathrm{~min}$ in an Eppendorf Mini Spin mini-centrifuge (Eppendorf, Westbury, NY). After the 30 min incubation and pre-wash, the solution containing GSTA1-1 and EdAG was filtered by centrifugation at 10,000 rpm for $45 \mathrm{~min}$. The enzyme was recovered by reconstitution with 100 $\mu \mathrm{L}$ of $100 \mathrm{mM}$ Tris buffer followed by a 30 min wash filtration of $100 \mu \mathrm{L}$ of $\mathrm{H}_{2} \mathrm{O}$. To the recovered enzyme in buffer, GSH and CDNB (final concentrations $1 \mathrm{mM}$ ) were added and diluted with water to a final volume of $2 \mathrm{~mL}$. The reaction was monitored for 30 minutes to assess enzyme activity by monitoring the change in UV absorbance at $340 \mathrm{~nm}$. Positive controls were incubated with $10 \mathrm{mM}$ ethacrynic acid (EA). Negative controls were incubated without EdAG, filtered and reconstituted with substrates, and velocities over 30 minutes were normalized to $100 \%$ recovered enzyme activity. EdAG concentrations during pre-incubations were 0.01 , $0.1,1.0$, and $10 \mathrm{mM}$. Experiments were performed in triplicate and the average percent recoveries calculated. The means \pm standard error of the means (SEM) were plotted and significance was determined using a two-tailed, unpaired Student's t-test, with $p<0.05$ as statistically significant. 


\subsubsection{Proteomic analysis of the GSTA1-1/EdAG adduct}

In order for EdAG to form a covalent adduct with GSTA1-1, pure GSTA1-1 (1.8 $\mu \mathrm{M})$ was mixed with $10 \mathrm{mM}$ EdAG for one hour in $10 \mathrm{mM}$ Tris buffer, $\mathrm{pH}$ 7.4. Excess, unreacted EdAG was removed by filtration, as described above, and reconstituted in $80 \mu \mathrm{L}$ of water. Matrix-assisted laser desorption ionization-time of flight (MALDI-TOF) mass spectrometric analysis in the positive ion mode was used to determine if GSTA1-1 experienced a mass shift of 273 Da, equivalent to the mass addition of one EdAG molecule. A control of unreacted GSTA11 was used to calibrate the MALDI MS signal at 25,730 Da.

To elucidate the potential residue of GSTA1-1 that is conjugated to EdAG, a trypsin digestion was performed. Again, 1.8 $\mu \mathrm{M}$ GSTA1-1 was incubated with $10 \mathrm{mM}$ EdAG for one hour in $10 \mathrm{mM}$ Tris buffer, $\mathrm{pH}$ 7.4, followed by filtration to remove excess unreacted EdAG. The GSTA1-1 was reconstituted from the filters with $80 \mu \mathrm{L}$ of $100 \mathrm{mM}$ ammonium bicarbonate buffer, pH 8.6 in order to optimize enzyme conditions for the trypsin digest. A $1 \mathrm{mg} / \mathrm{mL}$ solution of trypsin was made in deionized water adjusted to $\mathrm{pH} 3.0$ with $10 \mathrm{mM} \mathrm{HCl}$. A 1/20 (w/w) addition of trypsin/GSTA1-1 was made to the reconstituted GSTA1-1 in $100 \mathrm{mM}$ $\mathrm{NH}_{4} \mathrm{HCO}_{3}$ and incubated in a $37^{\circ} \mathrm{C}$ water bath overnight for 18 hours. The samples were mixed with $\alpha$-cyano-4-hydroxycinnamic acid as matrix (1:1, v:v), spotted and dried on MALDI plate prior to analysis by a Micromass Reflectron MALDI-TOF mass spectrometer in the positive ion mode. A protein BLAST search was performed to list expected peptides from tryptic digestion. The peptide containing the cysteine residue will be monitored in control and with EdAG to detect any modifications of that cysteine by EdAG. 


\section{RESULTS}

\subsubsection{Inhibition of GSTs in Human Liver Cytosol by EdAG}

The inhibitory effects of EdAG were first evaluated using soluble GST isoforms in human liver cytosol by varying GSH and EdAG concentrations over a 100-fold range. The data are consistent with enzyme inhibition or inactivation involving mechanisms other than simple competitive processes. Figure 4.3.1 displays the Michaelis-Menton plot that suggests either irreversible or noncompetitive inhibition. Human liver cytosol provided an in vitro model of in vivo GST activity in liver.

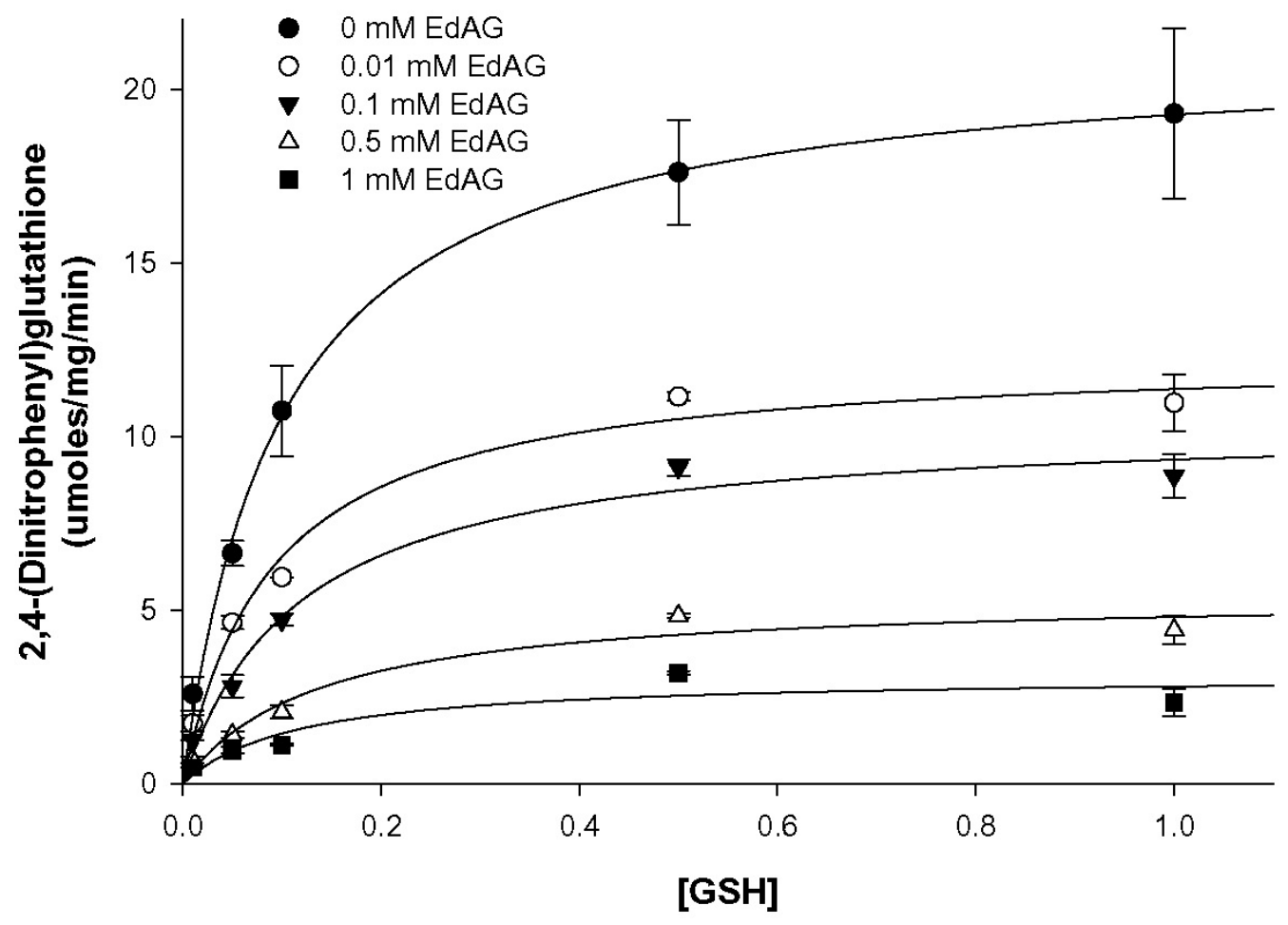

Figure 4.3.1 GST inhibition by EdAG 


\subsubsection{Inhibition of GSTA1-1 by EdAG}

To determine the effect of EdAG on the major isoform of GST associated with EdAG formation, inhibition studies with pure recombinant human GSTA1-1 were performed. Under the same incubation conditions as for the cytosolic fraction experiments, recombinant human GSTA1-1 was inhibited by EdAG in a manner that suggested either irreversible or noncompetitive inhibition. The mechanism of inhibition as noncompetitive is in accordance with the previous experiment involving all cytosolic isoforms of GSTs in human liver cytosol. The apparent $\mathrm{K}_{\mathrm{M}}$ and apparent $\mathrm{V}_{\mathrm{MAX}}$ for the reaction shown in Table 4.1 provide a calculated $\mathrm{IC}_{50}$ value of $99.2 \mu \mathrm{M}$ for inhibition of GSTA1-1 by EdAG.

\begin{tabular}{|l|l|l|l|l|l|}
\hline [EdAG] (mM) & $\mathbf{0}$ & $\mathbf{0 . 0 1}$ & $\mathbf{0 . 1}$ & $\mathbf{0 . 5}$ & $\mathbf{1}$ \\
\hline $\mathbf{K}_{\mathbf{M}}{ }^{\text {app }}(\mu \mathbf{M})$ & 85.8 & 131 & 177 & 67.8 & 79.7 \\
\hline $\begin{array}{l}\mathbf{V}_{\mathbf{M A X}}{ }^{\text {app }} \\
(\mu \mathbf{m o l e s} / \mathbf{m g} / \mathbf{m i n})\end{array}$ & 40.1 & 40.8 & 19.1 & 6.21 & 6.34 \\
\hline
\end{tabular}

Table 4.1 Enzyme Kinetic Parameters for GSTA1-1 Inhibition by EdAG Support Mixed Noncompetitive Inhibition

The results were not consistent with competitive inhibition at the G-site. The data were better explained by a complex inhibitory mechanism, as likely to be expected for a two-substrate enzyme, such as GST. The data support mixed noncompetitive inhibition based on unequal values given for both $\mathrm{K}_{\mathrm{IC}}(523 \mu \mathrm{M})$ and $\mathrm{K}_{\mathrm{IU}}(252 \mu \mathrm{M})$, respectively representing binding constants for competitive and uncompetitive inhibitors (22). The Michaelis-Menton plot is 
pictured in Figure 4.3.2 that suggests either irreversible or noncompetitive inhibition of GSTA11 by EdAG.

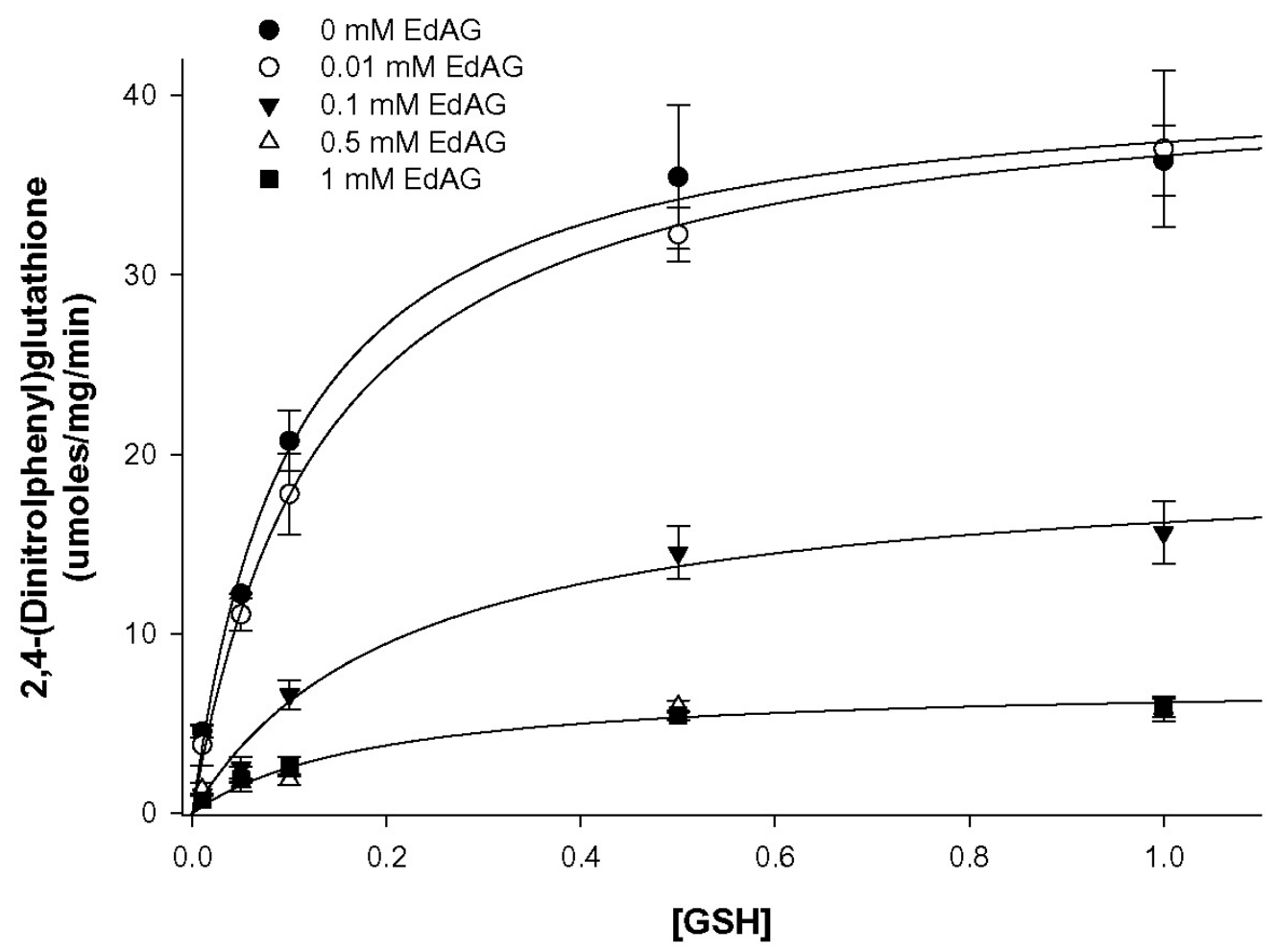

Figure 4.3.2 GSTA1-1 inhibition by EdAG

To further suggest noncompetitive inhibition, other kinetic diagrams were plotted such as Lineweaver-Burk (Figure 4.3.3), and Hanes-Woolf (Figure 4.3.4) plots, that both suggested noncompetitive inhibition based on the estimated extrapolated intersection point of the lines in the negative $\mathrm{x}$-axis and the unparallel lines. The slopes of both plots are equivalent to the inverse of $\mathrm{V}_{\mathrm{MAX}}$, and because $\mathrm{V}_{\mathrm{MAX}}$ is changing, so are the slopes. 


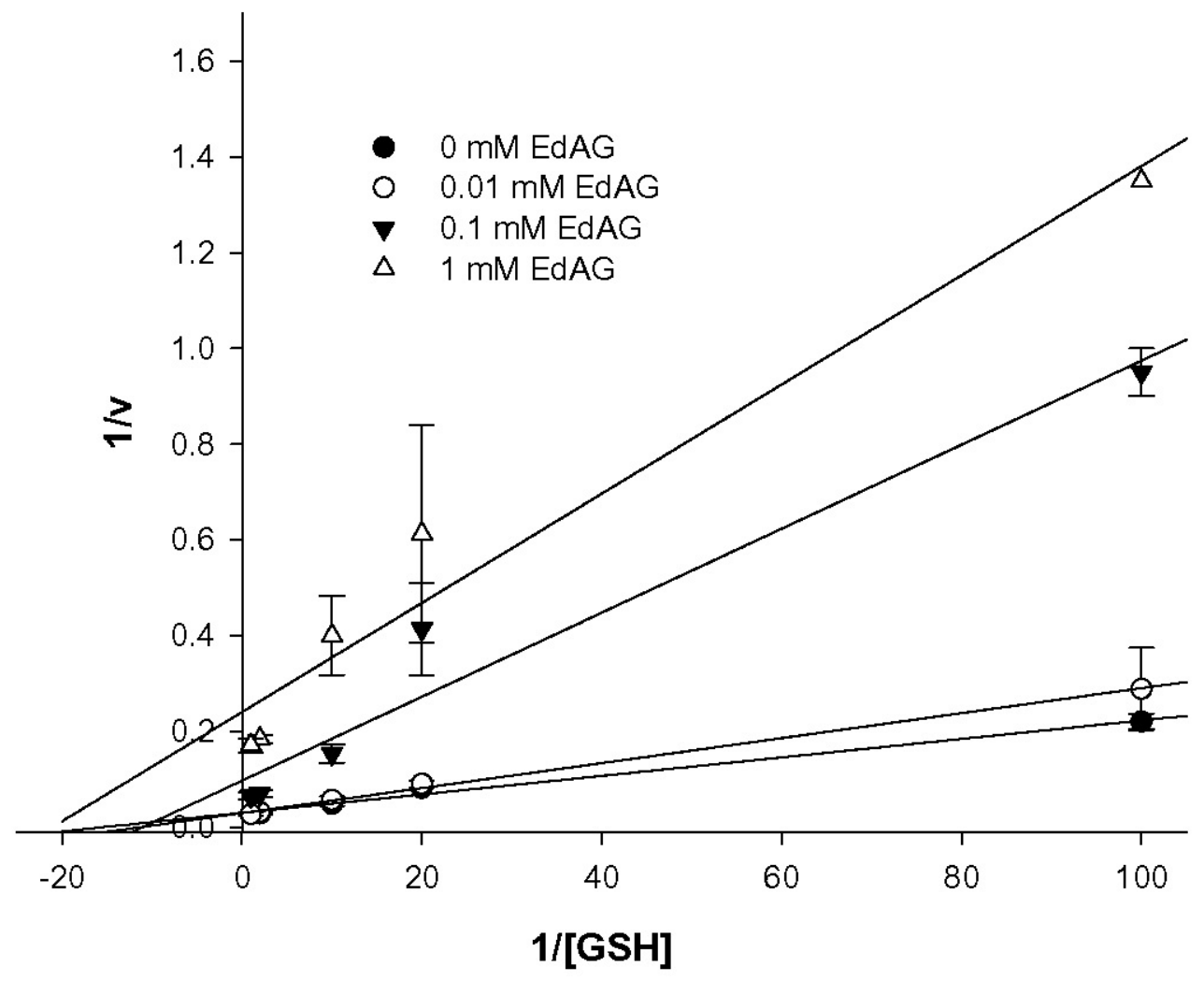

Figure 4.3.3 Lineweaver-Burke plot suggests noncompetitive inhibition of GSTA1-1 by EdAG 


\section{Hanes-Woolf Plot}

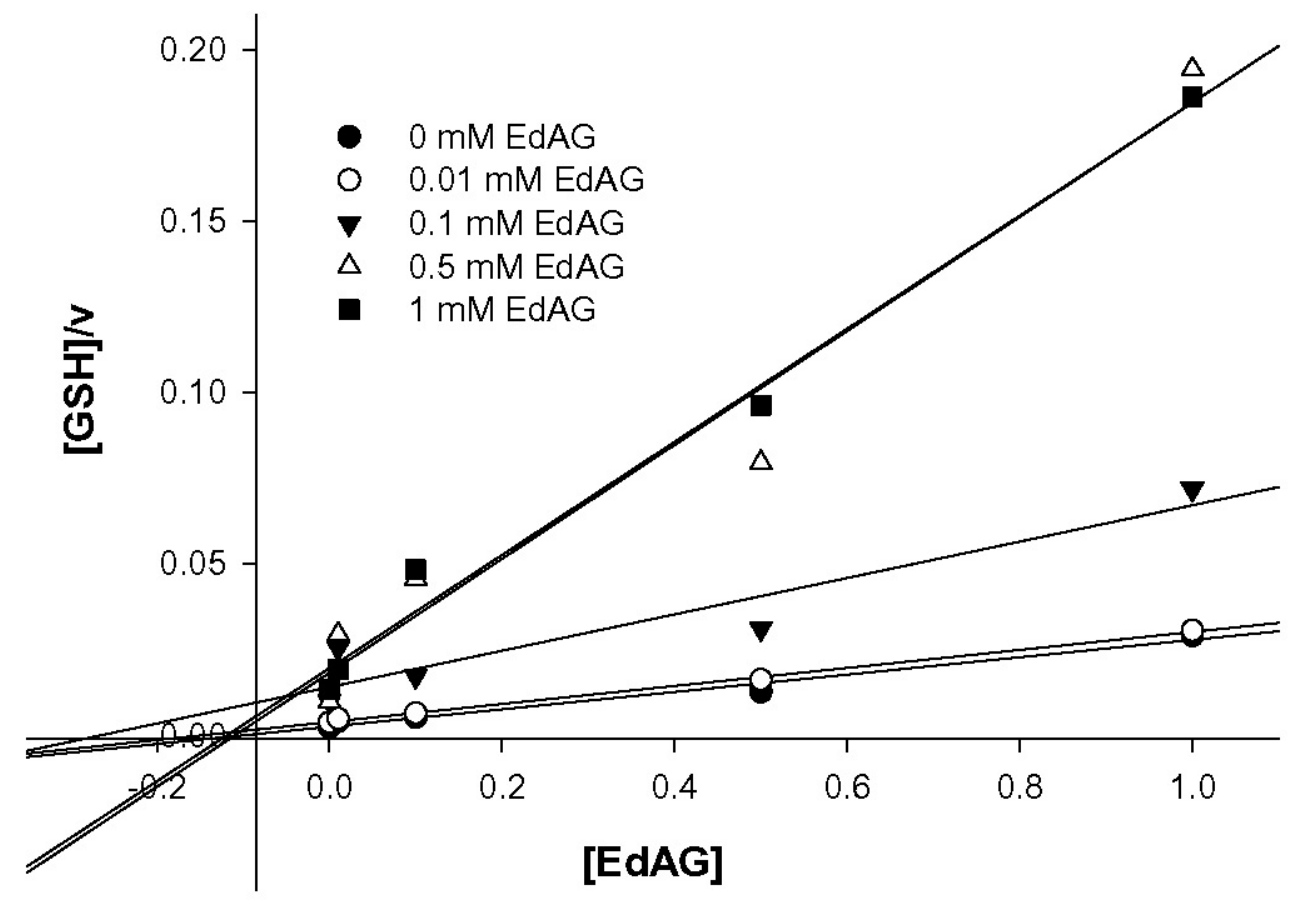

Figure 4.3.4 Hanes-Woolf plot suggests noncompetitive inhibition of GSTA1-1 by EdAG

To determine if there is a preference for inhibition at the G- or H-site of GSTA1-1, the enzyme assay was repeated holding GSH concentration constant at saturating levels (1.0 mM) and varying concentrations of CDNB. Concentrations of EdAG near the $\mathrm{IC}_{50}$ of $99.2 \mu \mathrm{M}$ displayed negligible decreases in velocity, suggesting that EdAG binding was not occurring at the H-site (Figure 4.3.5). The decrease in velocity when EdAG was $1 \mathrm{mM}$ results from the same GST inhibition observed in Figure 4.3.2, when all substrates and inhibitor were $1 \mathrm{mM}$. This experiment strongly suggested that EdAG is binding a separate non-substrate binding, or 
ligandin, site that is neither the G-site where GSH binds nor the H-site where CDNB and other hydrophobic xenobiotics bind.

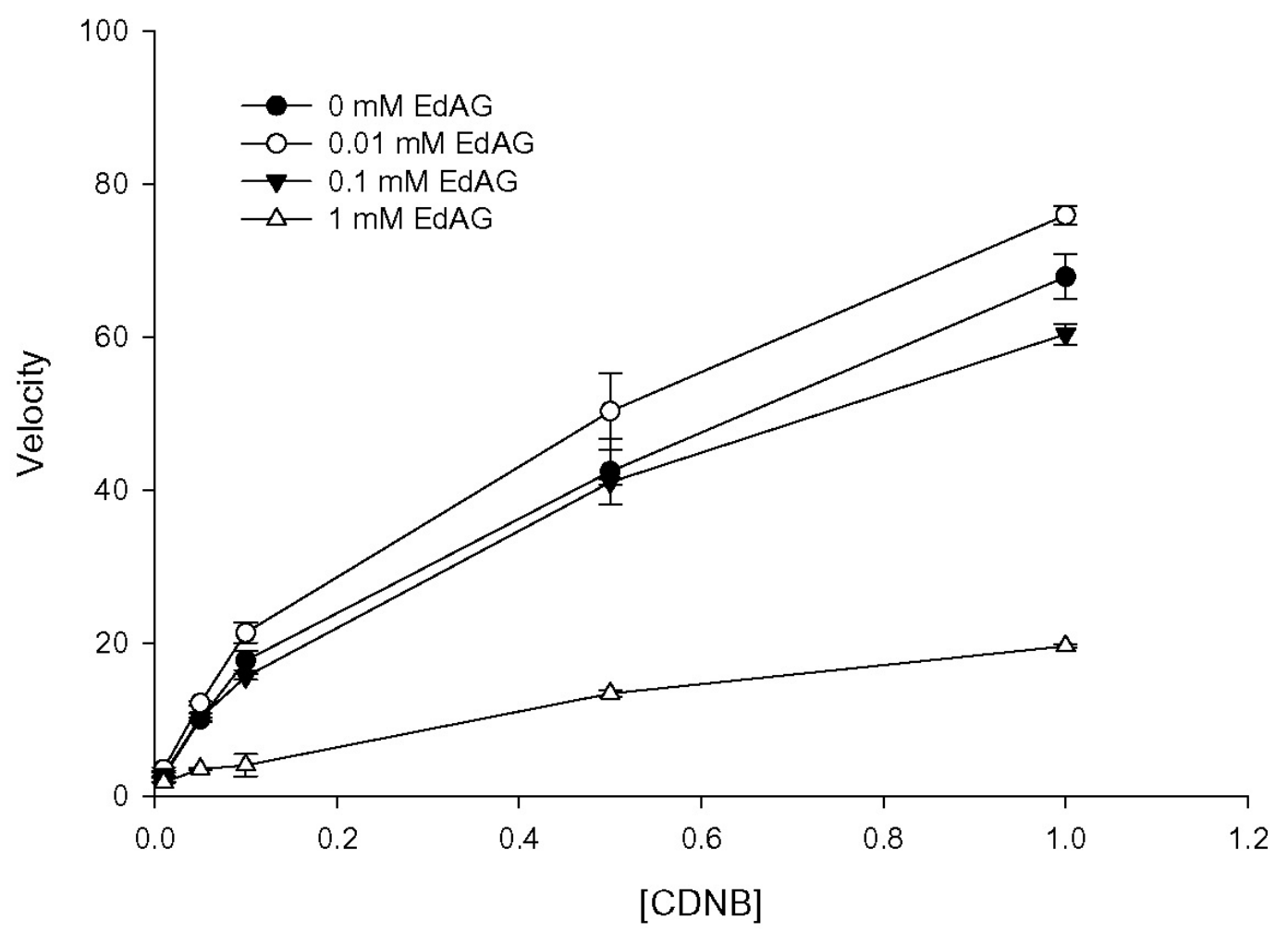

Figure 4.3.5 EdAG not inhibiting at substrate binding site

\subsubsection{Irreversible Inhibition of GST by EdAG}

The potential for irreversible inhibition of GST by EdAG was evaluated. The MichaelisMenton kinetic profiles of noncompetitive and irreversible inhibitors are similar in that inhibition is concentration-dependent and enzyme activity cannot be restored by increasing substrate (GSH) concentration. To determine if EdAG is also an irreversible inhibitor, EdAG was incubated at room temperature with GSTA1-1 in the absence of substrates to allow for 
irreversible binding to occur. Figure 4.3.6 shows that a 30-minute preincubation of EdAG at 10 mM concentration with GSTA1-1 in the absence of GSH and CDNB showed irreversible inhibition. Inhibition by preincubation concentrations at or below $1 \mathrm{mM} \mathrm{EdAG} \mathrm{was} \mathrm{not}$ observed after 30 minutes. As a control for irreversible inhibition, preincubation of GSTA1-1 with ethacrynic acid $(10 \mathrm{mM})$ inactivated the enzyme irreversibly (23). Experiments were conducted that demonstrated the binding reaction was complete after 30 minutes and no further inhibition occurred with preincubation times up to 2 hours (data not shown). Preincubations in a $37^{\circ} \mathrm{C}$ water bath compared to at room temperature also did not affect binding efficiency. Each bar is the average percent recovered GSTA1-1 activity $(n=3) \pm$ SEM. A two-tailed, unpaired Student's t-test was used to calculate significant difference in $\%$ activity (*P value $<0.05$ for reactions containing $10 \mathrm{mM}$ EA versus $0.01-1 \mathrm{mM}$ EdAG was considered statistically significant). 


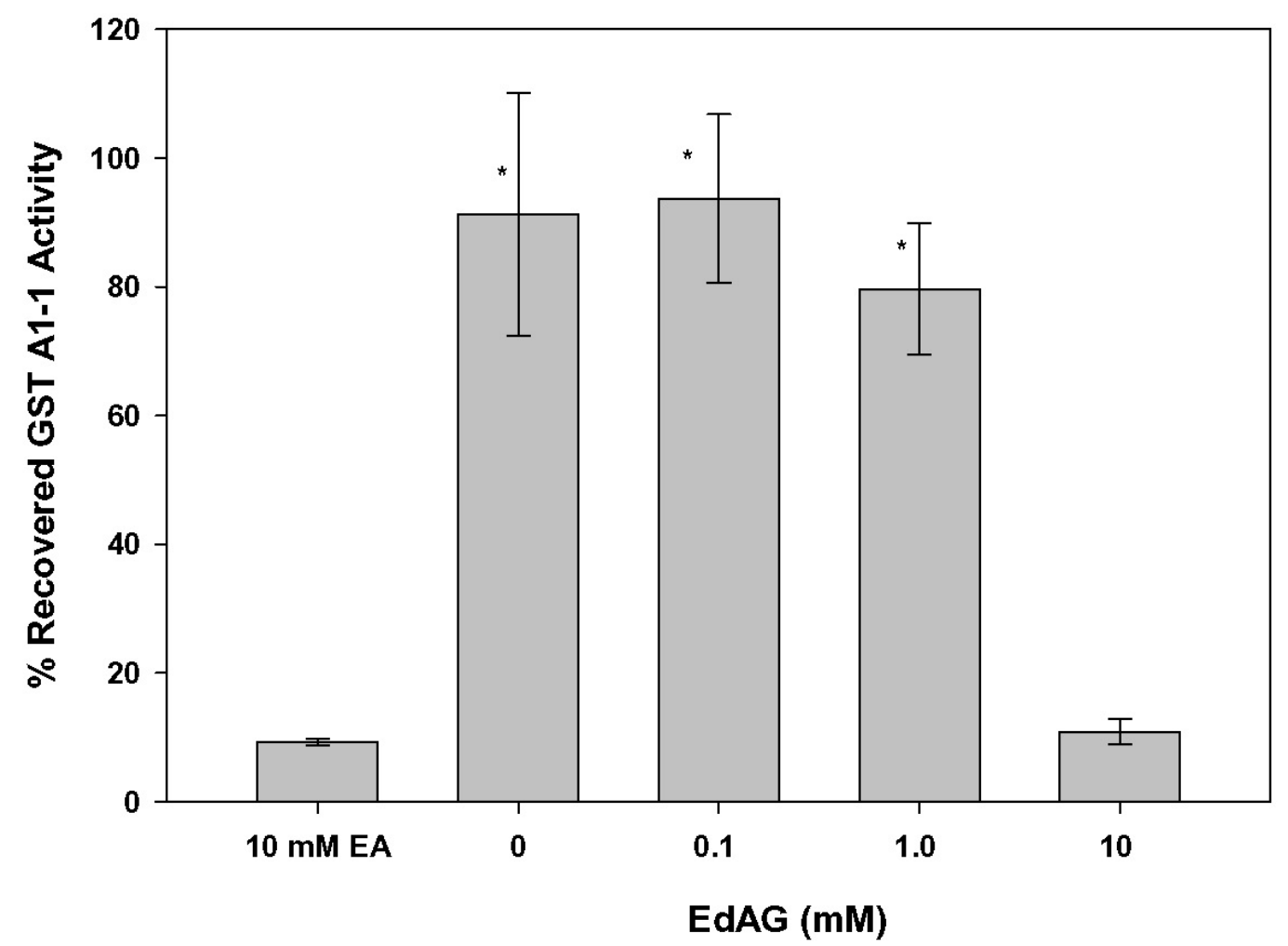

Figure 4.3.6 Irreversible inhibition of GSTA1-1 by EdAG and ethacrynic acid

Further experiments were performed to determine whether EdAG was covalently binding GSTA1-1. GSTA1-1 is a homodimer, with each subunit containing a cysteine residue at position 112. The most likely alkylation site is at a cysteine residue of GST. In the native homodimer, the two cysteines are located in the solvent-accessible cleft that forms between the two monomers. Figure 4.3.7 depicts the native conformation of the GSTA1-1 homodimer with the two cysteine residues (highlighted in green) courtesy of molecular modeling of the known crystal structure by John Jett. The position of these cysteine residues in the solvent cleft could determine the mechanism of GSTA1-1 irreversible inhibition by $10 \mathrm{mM} \mathrm{EdAG.} \mathrm{It} \mathrm{was} \mathrm{shown} \mathrm{in}$ Chapter 4 that EdAG is noncompetitively inhibiting GSTA1-1 at lower concentrations $(\leq 1 \mathrm{mM})$ 
at a site other than the catalytic sites (G-, H-site), and literature has demonstrated compounds that noncompetitively inhibit by binding at the solvent cleft $(16,17)$.

\subsubsection{Proteomic analysis of the GSTA1-1/EdAG adduct}

The irreversible inhibition of GSTA1-1 by $10 \mathrm{mM}$ EdAG was further characterized to determine if EdAG, an electrophilic compound previously shown to be conjugated by cysteine residues in insulin and albumin, is being conjugated by one or both of the cysteine residues in the solvent cleft. GSTA1-1 was preincubated with $10 \mathrm{mM}$ EdAG for up to one hour before removal of excess EdAG and reconstitution of GSTA1-1 in water for analysis by MALDI looking for an intact total mass shift corresponding to EdAG adduction.

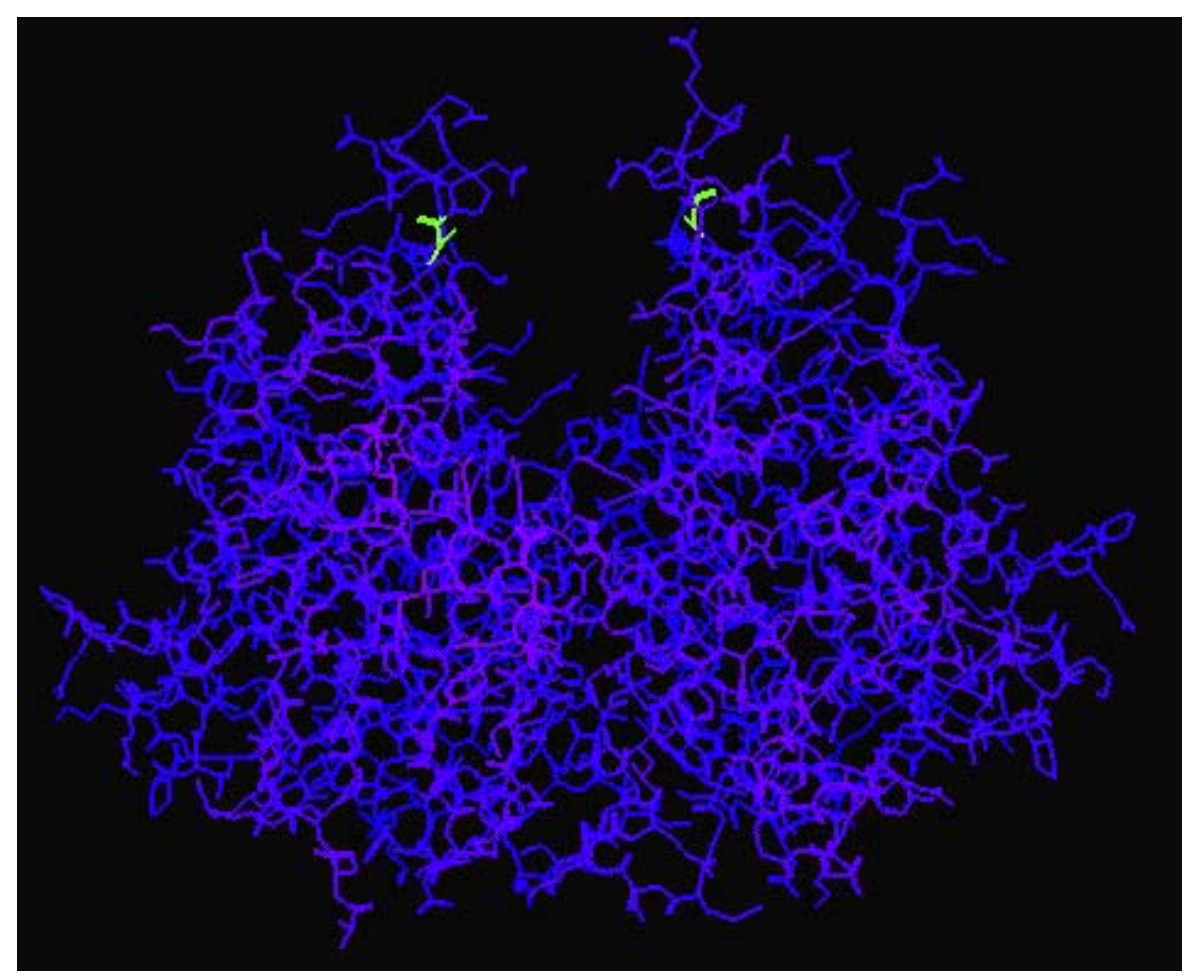

Figure 4.3.7 GSTA1-1 contains two cysteine residues in the solvent cleft 
Compared to control GSTA1-1, no mass shift was detected by MALDI for incubations of GSTA1-1 with $\leq 1 \mathrm{mM}$ EdAG, which was expected. However, at $10 \mathrm{mM}$ EdAG, a mass shift of 400+ Da was detected. The exact implications of this shift were not entirely clear, yet it corresponds with Figure 4.3.6 that only the $10 \mathrm{mM}$ EdAG incubation irreversibly inhibited GSTA1-1.

To more fully characterize a potential alkylation of EdAG by GSTA1-1, a trypsin digestion was performed prior to analysis by a Micromass Reflectron MALDI-TOF. Roughly 60 $\%$ of the expected tryptic peptides of GSTA1-1 were detected, but not the peptide containing the cysteine residue at position 112. Thus, it was not possible to elucidate whether EdAG was covalently adducted to Cys112. In the sample containing $10 \mathrm{mM}$ EdAG, the same GSTA1-1 tryptic peptides were detected as in control, indicating that EdAG was not conjugated to any other residues. 


\section{DISCUSSION}

\section{4}

The metabolism of the chemotherapeutic agent busulfan results in the formation of a dehydroalanine analog of glutathione (EdAG) that contains an $\alpha, \beta$-unsaturated system and renders the molecule an electrophilic Michael acceptor. Although dehydroalanines are relatively weak Michael acceptors because enamine intermediates contribute electron density to the electrophilic site (24), EdAG does react with the Michael donors GSH and cysteine, forming a thioether or lanthionine bond (19), as well as cysteine residues on insulin B-chain and serum albumin, previously demonstrated in Chapter 3 of this dissertation. The reactivity of EdAG toward proteins was extended to GSTs, which were previously indicated to bind GSTs and the mechanism of inhibition from binding of EdAG was characterized.

GST transfers glutathione to electrophilic substrates by a ternary complex mechanism (25). EdAG is an electrophilic compound that has been shown to bind GSTA1-1 (19). EdAG is not a substrate for GST, yet acts as a GST inhibitor. When incubated with human liver cytosolic fraction or pure recombinant human GSTA1-1, micromolar concentrations of EdAG demonstrated mixed noncompetitive inhibition, with a predominantly uncompetitive mechanism due to the value of $\mathrm{K}_{\mathrm{IU}}$ being lower than $\mathrm{K}_{\mathrm{IC}}$ as calculated by SigmaPlot. The apparent $\mathrm{V}_{\mathrm{MAX}}$ decreased substantially in the presence of increasing concentrations of EdAG, while the apparent $\mathrm{K}_{\mathrm{M}}$ values varied relatively less (Table 4.1), further indicating noncompetitive inhibition. Mixed noncompetitive inhibition was suggested based on the observation of changes in both the apparent $\mathrm{V}_{\mathrm{MAX}}$ and apparent $\mathrm{K}_{\mathrm{M}}$. If only apparent $\mathrm{K}_{\mathrm{M}}$ changes, the mechanism is considered simple competitive inhibition, and if only apparent $\mathrm{V}_{\mathrm{MAX}}$ changes, its pure noncompetitive. 
When both apparent $\mathrm{V}_{\mathrm{MAX}}$ and $\mathrm{K}_{\mathrm{M}}$ values change, it is considered mixed-type inhibition, and because apparent $V_{\mathrm{MAX}}$ values changed significantly more than apparent $\mathrm{K}_{\mathrm{M}}$, the term mixed noncompetitive inhibition was appropriate. Furthermore, when the values calculated for $\mathrm{K}_{\mathrm{IC}}$ and $\mathrm{K}_{\mathrm{IU}}$ (binding constants of competitive and uncompetitive inhibitors, respectively) are both calculated by SigmaPlot and are equal, the reaction is classified as pure noncompetitive (22). Since $\mathrm{K}_{\mathrm{IC}}$ was calculated to be greater than $\mathrm{K}_{\mathrm{IU}}$, EdAG exhibited predominantly uncompetitive inhibition, which in this instance indicated that EdAG was binding at a non-catalytic binding site, or ligandin site.

One explanation for the mixed noncompetitive inhibition is the suggested participation of a separate inhibitor binding site of higher affinity for EdAG than that of the G-site or H-site. The solvent cleft formed between the two subunits of the homodimeric GSTA1-1 could serve as the non-catalytic binding site for EdAG, which is supported by literature $(16-18,26)$. From these experiments, it is not clear whether multiple binding sites and multiple substrates are participating in the overall reaction. Another explanation of the complex mechanism of GSTA1-1 inhibition by EdAG was irreversible binding. Michaelis-Menton plots of noncompetitive and irreversible inhibition look similar. Noncompetitive inhibition was initially proposed due to the Lineweaver-Burk and Hanes-Woolf plots that both suggested mixed noncompetitive inhibition by the changing slopes and the location of the intersection of the bestfit lines in the negative $\mathrm{x}$-axis. Irreversibility experiments were performed nonetheless to rule it out and further support the mixed noncompetitive inhibition mechanism at concentrations at or less than $1 \mathrm{mM}$ EdAG.

GSTA1-1 was preincubated with various concentrations of EdAG (1000-fold concentration range) for 30 minutes, which is ample time for covalent or irreversible binding to 
occur. At micromolar EdAG concentrations, GSTA1-1 was not irreversibly inhibited, although minimal irreversible inhibition occurred (10-20\%), which could also be attributed to less enzyme protein recovery. At higher concentrations than expected for physiological conditions, millimolar concentrations of EdAG (10 mM) irreversibly inactivated GSTA1-1, presumably through covalent bond formation. EdAG concentrations of $1 \mathrm{mM}$ showed more irreversible inhibition than micromolar concentrations, but not to the same extent as the $10 \mathrm{mM}$ EdAG or the $10 \mathrm{mM}$ ethacrynic acid positive control, a known irreversible inhibitor of GSTs (23). The EdAG concentration of $10 \mathrm{mM}$ irreversibly inhibited GSTA1-1 to the same extent as the ethacrynic acid control. This suggested that at lower concentrations, EdAG demonstrates reversible mixed noncompetitive inhibition and at concentrations at or above $10 \mathrm{mM}$, EdAG demonstrates irreversible inhibition.

EdAG is a reactive electrophile previously shown in this dissertation to be conjugated by cysteine residues on proteins such as insulin B-chain and serum albumin. GSTA1-1 is irreversibly inactivated by high concentrations of EdAG and is suggested to be covalently bound. Because cysteine residues are the most nucleophilic, thus most likely, residue to conjugate EdAG, it was hypothesized that EdAG was bound to a cysteine residue in GSTA1-1. The GSTA1-1 homodimer contains only two cysteine residues out of a total of 444 residues, with one on each subunit at position 112. The published crystal structure of GSTA1-1 was modeled and showed that the two cysteine residues reside in the solvent cleft (Figure 4.3.7), where EdAG is suggested to interact with GSTA1-1. High mass MALDI mass spectrometry was performed and detected a mass shift in the intact GSTA1-1 when incubated with $10 \mathrm{mM}$ EdAG. Incubations with less than $10 \mathrm{mM}$ did not register a mass shift for GSTA1-1 by MALDI. When digested with trypsin, the peptide containing the cysteine residue was not detected by a Reflectron 
MALDI-TOF, thus it could not be concluded whether EdAG was covalently bound to Cys 112 . An alternative hypothesis for the reversible inhibition at lower EdAG concentrations and irreversibly at $10 \mathrm{mM} \mathrm{EdAG}$ is that EdAG is binding tightly but not covalently. Thus, at high concentrations (nonphysiological), the noncovalent interactions are strong enough to irreversibly inhibit GSTA1-1.

It is known that when GSH is not bound in the G-site, located in the C-terminal domain of GSTA1-1, the native homodimer is suggested to assume numerous conformations (27-29), although this has not been demonstrated through crystal structures. The propensity of GSH to bind in the G-site ultimately drives GSTA1-1 to assume its normal homodimeric native conformation that has been crystallized. When substrates are not bound, the C-terminal domain in the apo GSTA1-1, which is mostly $\alpha$-helical, can shift such that the G-site is more accessible to GSH (30). The C-terminal can ultimately denature, however the favored binding of GSH can slow the denaturing of the overall quaternary structure by stabilizing the C-terminal $\alpha$-helix $(6,31)$. When a ligandin, or non-substrate, is bound in the solvent cleft, this can alter the Cterminal domain that governs access to GSH binding to the G-site (6). If EdAG is noncovalently, but tightly binding in the solvent cleft, this could be preventing GSH from binding the G-site, thus causing irreversible inhibition of GSTA1-1.

In conclusion, it was demonstrated in this chapter that the electrophilic dehydroalanine analog of glutathione, EdAG, induces a concentration-dependent inhibition of GSTA1-1 activity. At concentrations at or below $1 \mathrm{mM}$, the mechanism is reversible mixed noncompetitive, but above $1 \mathrm{mM}$, EdAG irreversibly inactivates GSTA1-1. Since EdAG inhibits GST-mediated busulfan metabolism and is a metabolite of the GSH adduct of busulfan, it is suggested that product inhibition kinetics could influence busulfan pharmacokinetics. With a calculated $\mathrm{IC}_{50}$ of 
99.2 $\mu \mathrm{M}$ of EdAG for GSTA1-1, physiological concentrations of EdAG could induce GST inhibition and prolong the presence of busulfan in the liver, potentially causing hepatotoxicity. 


\section{REFERENCES}

(1) Board, P. G., Coggan, M., Chelvanayagam, G., Easteal, S., Jermiin, L. S., Schulte, G. K., Danley, D. E., Hoth, L. R., Griffor, M. C., Kamath, A. V., Rosner, M. H., Chrunyk, B. A., Perregaux, D. E., Gabel, C. A., Geoghegan, K. F., and Pandit, J. (2000) Identification, characterization, and crystal structure of the Omega class glutathione transferases. J. Biol. Chem. 275(32), 24798-24806.

(2) Mannervik, B. (1985) The isoenzymes of glutathione transferase. Adv. Enzymol. Relat Areas Mol. Biol. 57, 357-417.

(3) Pemble, S. E., Wardle, A. F., and Taylor, J. B. (1996) Glutathione S-transferase class Kappa: characterization by the cloning of rat mitochondrial GST and identification of a human homologue. Biochem. J. 319 ( Pt 3), 749-754.

(4) Board, P. G., Coggan, M., Wilce, M. C., and Parker, M. W. (1995) Evidence for an essential serine residue in the active site of the Theta class glutathione transferases. Biochem. J. 311 ( Pt 1), 247-250.

(5) Board, P. G., and Anders, M. W. (2005) Human glutathione transferase zeta. Methods Enzymol. 401, 61-77.

(6) Cameron, A. D., Sinning, I., L'Hermite, G., Olin, B., Board, P. G., Mannervik, B., and Jones, T. A. (1995) Structural analysis of human alpha-class glutathione transferase A1-1 in the apo-form and in complexes with ethacrynic acid and its glutathione conjugate. Structure. 3(7), 717-727.

(7) Oakley, A. J., Lo, B. M., Battistoni, A., Ricci, G., Rossjohn, J., Villar, H. O., and Parker, M. W. (1997) The structures of human glutathione transferase P1-1 in complex 
with glutathione and various inhibitors at high resolution. J. Mol. Biol. 274(1), 84100.

(8) Bjornestedt, R., Stenberg, G., Widersten, M., Board, P. G., Sinning, I., Jones, T. A., and Mannervik, B. (1995) Functional significance of arginine 15 in the active site of human class alpha glutathione transferase A1-1. J. Mol. Biol. 247(4), 765-773.

(9) Graminski, G. F., Kubo, Y., and Armstrong, R. N. (1989) Spectroscopic and kinetic evidence for the thiolate anion of glutathione at the active site of glutathione Stransferase. Biochemistry 28(8), 3562-3568.

(10) Kong, K. H., Takasu, K., Inoue, H., and Takahashi, K. (1992) Tyrosine-7 in human class Pi glutathione S-transferase is important for lowering the pKa of the thiol group of glutathione in the enzyme-glutathione complex. Biochem. Biophys. Res. Commun. 184(1), 194-197.

(11) Czerwinski, M., Gibbs, J. P., and Slattery, J. T. (1996) Busulfan conjugation by glutathione S-transferases alpha, mu, and pi. Drug Metab Dispos. 24(9), 10151019.

(12) Hayes, P. C., Harrison, D. J., Bouchier, I. A., McLellan, L. I., and Hayes, J. D. (1989) Cytosolic and microsomal glutathione S-transferase isoenzymes in normal human liver and intestinal epithelium. Gut 30(6), 854-859.

(13) Hou, L., Honaker, M. T., Shireman, L. M., Balogh, L. M., Roberts, A. G., Ng, K. C., Nath, A., and Atkins, W. M. (2007) Functional promiscuity correlates with conformational heterogeneity in A-class glutathione S-transferases. J. Biol. Chem. 282(32), 23264-23274. 
(14) Le, T., I, Stenkamp, R. E., Ibarra, C., Atkins, W. M., and Adman, E. T. (2002) 1.3-A resolution structure of human glutathione S-transferase with S-hexyl glutathione bound reveals possible extended ligandin binding site. Proteins 48(4), 618-627.

(15) Mahajan, S. S., Hou, L., Doneanu, C., Paranji, R., Maeda, D., Zebala, J., and Atkins, W. M. (2006) Optimization of bivalent glutathione S-transferase inhibitors by combinatorial linker design. J. Am. Chem. Soc. 128(26), 8615-8625.

(16) Bhargava, M. M., Listowsky, I., and Arias, I. M. (1978) Ligandin. Bilirubin binding and glutathione-S-transferase activity are independent processes. J. Biol. Chem. 253(12), 4112-4115.

(17) Jagt, D. L., Wilson, S. P., Dean, V. L., and Simons, P. C. (1982) Bilirubin binding to rat liver ligandins (glutathione S-transferases A and B). Relationship between bilirubin binding and transferase activity. J. Biol. Chem. 257(4), 1997-2001.

(18) Ohl, V. S., and Litwack, G. (1977) Selective inhibition of glutathione S-transferases by 17 beta-estradiol disulfate. Arch. Biochem. Biophys. 180(1), 186-190.

(19) Younis, I. R., Elliott, M., Peer, C. J., Cooper, A. J., Pinto, J. T., Konat, G. W., Kraszpulski, M., Petros, W. P., and Callery, P. S. (2008) Dehydroalanine analog of glutathione: an electrophilic busulfan metabolite that binds to human glutathione S-transferase A1-1. J. Pharmacol. Exp. Ther. 327(3), 770-776.

(20) Habig, W. H., Pabst, M. J., and Jakoby, W. B. (1974) Glutathione S-transferases. The first enzymatic step in mercapturic acid formation. J. Biol. Chem. 249(22), 71307139.

(21) Dieckhaus, C. M., Roller, S. G., Santos, W. L., Sofia, R. D., and Macdonald, T. L. (2001) Role of glutathione S-transferases A1-1, M1-1, and P1-1 in the 
detoxification of 2-phenylpropenal, a reactive felbamate metabolite. Chem. Res. Toxicol. 14(5), 511-516.

(22) Nomenclature Committee of the International Union of Biochemistry (NC-IUB) (1983) Symbolism and Terminology in Enzyme Kinetics. Biochem.J. 213, 561-571.

(23) Ploemen, J. H., van, O. B., Bogaards, J. J., and van Bladeren, P. J. (1993) Ethacrynic acid and its glutathione conjugate as inhibitors of glutathione S-transferases. Xenobiotica 23(8), 913-923.

(24) Li, G., and van der Donk, W. A. (2007) Efficient synthesis of suitably protected betadifluoroalanine and gamma-difluorothreonine from L-ascorbic acid. Org. Lett. $9(1), 41-44$.

(25) Pabst, M. J., Habig, W. H., and Jakoby, W. B. (1974) Glutathione S-transferase A. A novel kinetic mechanism in which the major reaction pathway depends on substrate concentration. J. Biol. Chem. 249(22), 7140-7147.

(26) McTigue, M. A., Williams, D. R., and Tainer, J. A. (1995) Crystal structures of a schistosomal drug and vaccine target: glutathione S-transferase from Schistosoma japonica and its complex with the leading antischistosomal drug praziquantel. $J$. Mol. Biol. 246(1), 21-27.

(27) Nieslanik, B. S., Dietze, E. C., Atkins, W. M., Trong, I. L., and Adman, E. (1999) The locally denatured state of glutathione S-transferase A1-1: transition state analysis of ligand-dependent formation of the C-terminal helix. Pac. Symp. Biocomput., 554-565.

(28) Nieslanik, B. S., Dabrowski, M. J., Lyon, R. P., and Atkins, W. M. (1999) Stopped-flow kinetic analysis of the ligand-induced coil-helix transition in glutathione S- 
transferase A1-1: evidence for a persistent denatured state. Biochemistry 38(21), 6971-6980.

(29) Nieslanik, B. S., Ibarra, C., and Atkins, W. M. (2001) The C-terminus of glutathione Stransferase A1-1 is required for entropically-driven ligand binding. Biochemistry 40(12), 3536-3543.

(30) Gustafsson, A., Etahadieh, M., Jemth, P., and Mannervik, B. (1999) The C-terminal region of human glutathione transferase A1-1 affects the rate of glutathione binding and the ionization of the active-site Tyr9. Biochemistry 38(49), 1626816275.

(31) Sinning, I., Kleywegt, G. J., Cowan, S. W., Reinemer, P., Dirr, H. W., Huber, R., Gilliland, G. L., Armstrong, R. N., Ji, X., Board, P. G., and . (1993) Structure determination and refinement of human alpha class glutathione transferase A1-1, and a comparison with the Mu and Pi class enzymes. J. Mol. Biol. 232(1), 192212.

(32) DeLeve, L. D., and Wang, X. (2000) Role of oxidative stress and glutathione in busulfan toxicity in cultured murine hepatocytes. Pharmacology 60(3), 143-154. 


\section{Chapter 5}

\section{Reactivity of EdAG as a Radical Scavenger}




\section{INTRODUCTION}

\subsubsection{Dehydroalanines as free radical scavengers}

$\mathrm{N}$-Acylated dehydroalanines, of which EdAG is an example, have radical scavenging properties through a captodative mechanism (1). This unusual class of reactive oxygen species (ROS) scavengers involves carbon-based radical intermediates that differentiate captodative mechanisms from the scavenging properties of GSH, $N$-acetylcysteine, and ascorbic acid (2). GSH is the most abundant cellular thiol and plays a major role in ROS scavenging, as well as in transition metal transport due to the ability of the thiol moiety to donate a hydrogen atom with an electron pair (Figure 5.1.1).

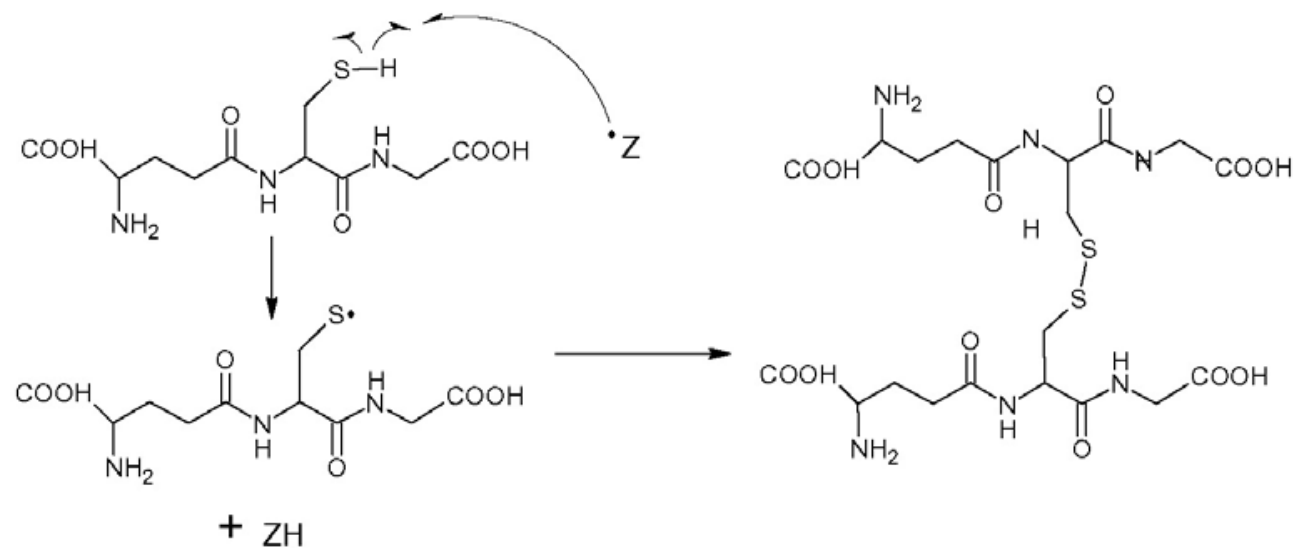

Figure 5.1.1 Glutathione redox mechanism for radicals

Amino acids and peptides that undergo a one-electron oxidation are examples of captodative stabilization of carbon-based radicals (3). The structural features that stabilize a captodative radical on the $\alpha$-carbon of a peptide are adjacent groups consisting of a lone pair-donating amide nitrogen and an electron-withdrawing carbonyl. Captodative free radical intermediates have 
been identified in the addition of hydroxyl radical to dehydroalanine compounds (4) as demonstrated in Figure 5.1.2.

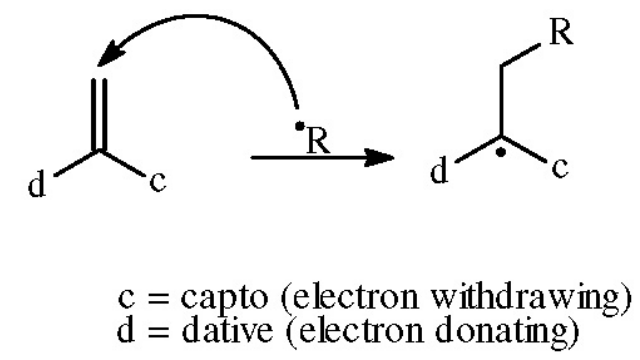

Figure 5.1.2 Captodative mechanism of radical scavenging by dehydroalanines

Dehydroalanine-containing compounds constitute an unusual class of radical scavengers that have been studied for the past few decades and the proposed mechanisms are different from redox mechanisms utilized by the more common reducing agents GSH, $N$-acetyl cysteine, and ascorbic acid. $N$-acyldehydroalanines contain both electron-withdrawing and electron-donating groups that form a captodative substitution system (5), which surrounds a central alpha carbon in a dehydroalanine amino acid residue. The carbonyl system serves as the electron-withdrawing group, allowing the $\beta$ carbon adjacent to the central $\alpha$ carbon to obtain a partial positive charge and make an electrophilic site for attack by ${ }^{\bullet} \mathrm{OH}$. The resulting free electron is then stabilized on the $\alpha$-carbon by captodative mechanisms, where the amide group adjacent to the $\alpha$ carbon is the electron-donating group and the carbonyl group adjacent to the $\alpha$ carbon is the electronwithdrawing group (Figure 5.1.2). The stabilized radical does not polymerize, but rather dimerizes with other stabilized radicals (6). There are numerous examples of low molecular weight compounds containing dehydroalanine moieties that are able to scavenge ROS such as hydroxyl $\left({ }^{\bullet} \mathrm{OH}\right)$ and superoxide $\left(\mathrm{O}_{2}^{-\bullet}\right)$ radical, as well as reducing lipid peroxidation $(1,4,7-13)$. 
Acyldehydroalanines reduce the amount of hydroxyl radical formed in the Fenton reaction, suggesting that these compounds could be successful as ROS scavengers in biological systems. Suzen et al synthesized eleven $N$-acyl dehydroalanine compounds to study their free radical scavenging properties by testing the protection against lipid peroxidation as well as scavenging of 1,1-diphenyl-2-picrylhydrazyl (DPPH) radical, where the dehydroalanine compounds protected against peroxidation but did not scavenge DPPH radical due to the lack of a redox thiol functional group as expected (12).

In this chapter, the dehydroalanine analog of glutathione, EdAG, was tested using the previously mentioned reactions (DPPH, Fenton reaction and EPR) to study carbon-based radical formation through hydroxyl radical trapping. Mass spectrometry was added to structurally identify the EdAG-hydroxyl radical adduct. 


\section{EXPERIMENTAL PROCEDURES}

\subsubsection{Materials}

GSH, Tris buffer, pH 7.4, 5,5-dimethyl-1-pyrroline- $N$-oxide (DMPO), $\alpha$-(4-pyridyl $N$ oxide)- $N$-tert-butylnitrone (POBN), 1,1-diphenyl-2-picrylhydrazyl (DPPH), $N$-acetylcysteine (NAC), Tris buffer, $\mathrm{pH}$ 7.4, PBS, serum free media (SFM), Dulbecco's modified eagle's medium (DMEM), fetal bovine serum (FBS), hydrogen peroxide $\left(\mathrm{H}_{2} \mathrm{O}_{2}\right)$, sodium dichromate, xanthine, xanthine oxidase, and ferrous sulfate $\left(\mathrm{FeSO}_{4}\right)$ were purchased from Sigma-Aldrich (St. Louis, MO). All water used was deionized with a NANOpure ${ }^{\circledR}$ water purification system (Barnstead, San Jose, CA). RAW 264.7 mouse monocyte macrophages and BEAS-2B bronchial epithelial cells were purchased from American Type Culture Collection (ATCC, Rockville, MD). The spin trap DMPO was purified by charcoal decolorization and vacuum distillation to remove any electron spin resonance (EPR)-detectable impurities.

\subsubsection{Instrumentation}

Ultraviolet (UV) absorbance was measured on a Beckman DU 640 UV/Vis spectrophotometer (Beckman-Coulter, Fullerton, CA). Electron paramagnetic resonance (EPR) (or electron spin resonance, EPR) was performed on a Bruker EMX spectrometer (Bruker Instruments, Billerica, MA) with a flat-cell assembly. High-performance liquid chromatography (HPLC) was performed on a Shimadzu 10 Avp binary pump HPLC system equipped with a UV/Vis detector (Shimadzu Scientific Instruments, Columbia, MD). Mass spectrometry was performed using an electrospray ionization (ESI) quadrupole ion trap (QIT) LCQ DECA (ThermoFisher Scientific, Waltham, MA), capable of multi-stage mass spectrometry (MS, 
$\mathrm{MS} / \mathrm{MS}, \mathrm{MS}^{3}$ ). Accurate mass analysis was performed on an ESI LTQ-FT (ThermoFisher, Waltham, MA).

\subsubsection{Reactivity of DPPH radical with GSH and EdAG}

The stable free radical $\mathrm{DPPH}^{\bullet}$ in methanol (final conc. $250 \mu \mathrm{M}$ ) was mixed with varying concentrations of GSH, $\mathrm{N}$-acetylcysteine (NAC), or EdAG (final methanol conc. was 50\%) based on literature procedures using DPPH (12). A control DPPH ${ }^{\bullet}$ solution in $1 \mathrm{mM}$ Tris buffer, pH 7.4 was used to determine baseline changes in absorbance at $515 \mathrm{~nm}$ over 30 minutes. Concentrations of GSH, NAC, and EdAG ranged from 0-500 $\mu \mathrm{M}$. Reduction of $\mathrm{DPPH}^{\bullet}$ to reduced DPPH was calculated using the extinction coefficient for DPPH ${ }^{\bullet}$ at $515 \mathrm{~nm}, 1.16 \times 10^{4} \mathrm{M}^{-}$

${ }^{1} \mathrm{~cm}^{-1}(2)$. Experiments were performed in duplicate and averaged to determine velocity of $\mathrm{DPPH}^{\bullet}$ to reduced DPPH.

\subsubsection{Formation of the EdAG Captodative Radical In Vitro}

Hydroxyl radical scavenging activity by EdAG was monitored by electron paramagnetic resonance (EPR) experiments conducted on a Bruker EMX spectrometer (Billerica, MA). Hydroxyl radical $\left({ }^{\bullet} \mathrm{OH}\right)$ was generated by the Fenton reaction containing $\mathrm{H}_{2} \mathrm{O}_{2}(0.1 \mathrm{mM})$ and $\mathrm{FeSO}_{4}(0.1 \mathrm{mM})$. The reaction mixture also contained either DMPO $(100 \mathrm{mM})$ or POBN (20 $\mathrm{mM})$ as spin traps, and the captodative radical scavenger EdAG $(0-50 \mathrm{mM})$ in a final volume of $300 \mu \mathrm{L}$ containing $\mathrm{PBS}, \mathrm{pH}$ 7.4. $\mathrm{H}_{2} \mathrm{O}_{2}$ was added last to initiate the formation of ${ }^{\bullet} \mathrm{OH}$ before the reaction was immediately transferred to a flat-cell for EPR analysis. As the short-lived hydroxyl radical is formed, it is immediately trapped by DMPO or POBN to obtain the DMPO-OH ${ }^{\bullet}$ or $\mathrm{POBN}-\mathrm{OH}^{\bullet}$ spin adduct, respectively, that is observed as a spin-trapped signal in the EPR. Note 
that only one spin trap (DMPO or POBN) is used in a run. Presence of other ${ }^{\bullet} \mathrm{OH}$-scavenging compounds will decrease that signal through competition for hydroxyl radical, and a rate constant of scavenging for EdAG $\left(\mathrm{k}_{\mathrm{E}}\right)$ can be calculated compared to that of DMPO $\left(\mathrm{k}_{\mathrm{D}}\right)$ as described previously $(14,15)$. Experiments were performed under ambient air at room temperature in triplicate, and the average values used to determine the rate of scavenging for $\operatorname{EdAG}\left(\mathrm{k}_{\mathrm{E}}\right)$ from the equation:

$\mathrm{V} / \mathrm{v}-1=\mathrm{k}_{\mathrm{E}}[\mathrm{EdAG}] / \mathrm{k}_{\mathrm{D}}[\mathrm{DMPO}]$, where $\mathrm{V}$ and $\mathrm{v}$ are the velocities of ${ }^{\bullet} \mathrm{OH}$ scavenging in the presence and absence of EdAG, respectively, and $\mathrm{k}_{\mathrm{E}}$ and $\mathrm{k}_{\mathrm{D}}$ represent the rate constant for hydroxyl radical scavenging by EdAG and DMPO, respectively. The literature value used for $\mathrm{k}_{\mathrm{D}}$ was $2.1 \times 10^{-9} \mathrm{M}^{-1} \mathrm{~s}^{-1}(16)$. POBN was used as a supplemental spin trap to draw further conclusions as to the mechanism of EdAG-hydroxyl radical scavenging, and not for rates of scavenging calculations. The mean \pm SEM were plotted for each concentration of EdAG and a two-tailed, unpaired Student's t-test was employed to determine statistical differences.

* Significance was determined with a $p<0.05$.

The scavenging ability of EdAG was qualitatively assessed against the superoxide radical $\left(\mathrm{O}_{2}{ }^{-\bullet}\right)$. Superoxide was generated by the xanthine $[3.5 \mathrm{mM}]$ and xanthine oxidase $[2.0 \mathrm{U} / \mathrm{ml}]$ system. A single concentration of $\operatorname{EdAG}(20 \mathrm{mM})$ was mixed with xanthine and xanthine oxidase and immediately transferred to a flat cell for EPR analysis.

\subsubsection{Mass spectrometric identification of EdAG-hydroxyl radical adduct}

A Fenton reaction using $2 \mathrm{mM}$ of both $\mathrm{H}_{2} \mathrm{O}_{2}$ and $\mathrm{FeSO}_{4}$ with either 5 or $10 \mathrm{mM} \mathrm{EdAG}$ was performed in a test tube and immediately analyzed by LC/MS. Controls of EdAG with only $\mathrm{Fe}_{2} \mathrm{SO}_{4}$ and $\mathrm{EdAG}$ with only $\mathrm{H}_{2} \mathrm{O}_{2}$ were run to ensure the desired product is strictly formed from 
the reaction of EdAG with hydroxyl radical formed by the Fenton reaction. HPLC conditions performed on a Shimadzu 10 AVP (Shimadzu Scientific, Columbia, MD) following an injection volume of $10 \mu \mathrm{L}$ were as follows: A Thermo Hybercarb column $(100 \times 2.1 \mathrm{~mm}, 5 \mu \mathrm{m}$, ThermoFisher, Waltham, MA) was used to isolate the product from other constituents in the reaction mixture. A gradient of mobile phase A $(0.5 \%$ formic acid in $90 / 10, \mathrm{v} / \mathrm{v}$, water/acetonitrile) and mobile phase B ( $0.1 \%$ formic acid in 50/50, v/v, isopropanol $/$ methanol) at $0.2 \mathrm{~mL} / \mathrm{min}$ was run from $0-5$ minutes using $100 \% \mathrm{~A}$; from $5-7$ minutes, $0 \rightarrow 50 \% \mathrm{~B}$; from $7-9$ minutes, $50 \rightarrow 0 \% \mathrm{~B}$; and from 9-15 minutes, $100 \% \mathrm{~A}$. The LCQ DECA mass spectrometer was operated in the positive ion full scan mode with a spray voltage of $5.2 \mathrm{kV}$, capillary voltage of 30 $\mathrm{V}$, capillary temperature of $300^{\circ} \mathrm{C}$, and a nitrogen sheath gas flow rate of 80 arbitrary units. Tandem mass spectrometry (MS/MS) was performed on the major product in the reaction mixture $(\mathrm{m} / \mathrm{z} 581.2)$ with an isolation width of $1.0 \mathrm{amu}$ and a percent collision energy of $30 \%$ on the LCQ DECA ion trap mass spectrometer.

\subsubsection{Accurate mass analysis of the ESG dimer}

Accurate mass calculations of the EdAG-OH (the serine analog of GSH, or ESG) dimer were performed on a LTQ-FT mass spectrometer (ThermoFisher, Waltham, MA) in the Fourier transfer (FT) mode. The LTQ-FT calculated a theoretical mass from the chemical formula $\mathrm{C}_{20} \mathrm{H}_{33} \mathrm{~N}_{6} \mathrm{O}_{14}$, consistent with protonated ESG dimer. The Fenton reaction consisting of $2 \mathrm{mM}$ of both $\mathrm{H}_{2} \mathrm{O}_{2}$ and $\mathrm{FeSO}_{4}$ was mixed with $10 \mathrm{mM}$ EdAG to produce the ESG dimer and directly injected into the LTQ-FT mass spectrometer for accurate mass analysis. The observed accurate mass obtained and its accuracy compared to the theoretical accurate mass was calculated. Mass differences of $5 \mathrm{ppm}$ or less from the theoretical mass are considered to be accurate. Tandem 
MS was performed in the FT mode on the LTQ-FT to obtain accurate mass calculations of the MS/MS fragment ions of the ESG dimer. The parent ion $m / z 581.20$ was fragmented with a collision-induced dissociation (CID) energy setting of 35 .

\subsubsection{Reaction of EdAG with Hydroxyl Radical ( $\left.{ }^{\circ} \mathrm{OH}\right)$ in RAW 264.7 Cells}

RAW 264.7 cells $\left(3 \times 10^{6} /\right.$ well) were cultured in DMEM over 24 hours in a $37^{\circ} \mathrm{C}$

incubator. The cells were then incubated with varying concentrations of EdAG for a period of 1 hour in a $37^{\circ} \mathrm{C}$ incubator. After 1 hour, the cells were transferred to a mini centrifuge tube, followed by the addition of potassium tetraperoxochromate (source of $\mathrm{Cr}[\mathrm{VI}]$ ) and DMPO to induce ROS production (specifically ${ }^{\circ} \mathrm{OH}$ ) and trap radicals for detection in EPR, respectively. The solution was mixed and quickly transferred to a flat cell for EPR analysis.

\subsubsection{EdAG Cell Permeability}

Six wells of 500,000 BEAS-2B (bronchial epithelial cells) were plated overnight in a 37 ${ }^{\circ} \mathrm{C}$ incubator in DMEM growth serum. Solutions of EdAG in PBS $(100,200$, and $500 \mu \mathrm{M})$ were aliquoted and $2 \mathrm{~mL}$ added to cells after $2 \mathrm{~mL}$ of fetal serum albumin (FBS) growth media was removed. EdAG was incubated with cells for 1 hour at $37{ }^{\circ} \mathrm{C}$. Afterwards, $1 \mathrm{~mL}$ of media was removed and transferred to a mini-centrifuge tube and centrifuged at 13,000 rpm for 15 minutes at $4{ }^{\circ} \mathrm{C}$. The supernatant, which contained EdAG that did not enter cell, was analyzed by LC/MS to quantify EdAG. The remaining media was removed from each well and replaced with $500 \mu \mathrm{L}$ of either deionized water (to 3 wells containing each EdAG concentration) or $0.05 \%$ digitonin (to other 3 wells) in order to lyse the cells. Cells were immediately scraped to detach them from the 
plate and the $500 \mu \mathrm{L}$ media was transferred to a mini-centrifuge tube where it sat for 30 minutes at room temperature to lyse the cell. After 30 minutes, tubes were centrifuged at 13,000 rpm for 15 minutes at $4{ }^{\circ} \mathrm{C}$ to removed cell fractions and obtain cytosol as supernatant. Samples were then analyzed by LC/MS to quantify EdAG in cytosol of cells. Chromatographic separation was achieved using a Thermo Hypercarb column (100 x $2.1 \mathrm{~mm}$ I.D., $5 \mu \mathrm{m}$ particle size)

(ThermoFisher, Waltham, MA). A gradient of mobile phase A ( $0.5 \%$ formic acid in $90 / 10$, v/v, water/acetonitrile) and mobile phase B ( $0.1 \%$ formic acid in 50/50, v/v, isopropanol/methanol) at $0.2 \mathrm{~mL} / \mathrm{min}$ was run from $0-5$ minutes using $100 \% \mathrm{~A}$; from $5-7$ minutes, $0 \rightarrow 50 \% \mathrm{~B}$; from $7-9$ minutes, $50 \rightarrow 0 \% \mathrm{~B}$; and from 9-15 minutes, $100 \% \mathrm{~A}$. The LCQ DECA mass spectrometer was operated in the positive ion full scan mode with a spray voltage of $5.2 \mathrm{kV}$, capillary voltage of 30 $\mathrm{V}$, capillary temperature of $300^{\circ} \mathrm{C}$, and a nitrogen sheath gas flow rate of 80 arb. units. The following ions were monitored: $m / z$ 274, and $m / z$ 308, corresponding to EdAG and GSH. GSH was monitored to determine if cells were effectively lysed. The presence of GSH in cell lysates after addition of digitonin confirmed the lysing of the cells. 


\section{RESULTS}

\subsubsection{Comparison of the reactivity of GSH, N-Acetylcysteine and EdAG with DPPH}

radical

In comparison to Suzen et al that demonstrated the inability of dehydroalanine-containing compounds to scavenge DPPH radical $\left(\mathrm{DPPH}^{\bullet}\right)(12), \mathrm{EdAG}$ was mixed with $\mathrm{DPPH}^{\bullet}$ to determine its redox capabilities. Reduced GSH and $N$-acetylcysteine in $100 \mathrm{mM}$ Tris buffer, $\mathrm{pH}$ 7.4, when mixed with a final concentration of $250 \mu \mathrm{M} \mathrm{DPPH}^{\bullet}$ in methanol, were effective in reducing the absorbance of $\mathrm{DPPH}^{\bullet}$ at $515 \mathrm{~nm}$. Presumably, GSH and $N$-acetylcysteine were oxidized to disulfides concomitantly with the conversion of $\mathrm{DPPH}^{\bullet}$ to reduced DPPH. When present in equimolar amounts to $\mathrm{DPPH}^{\bullet}, \mathrm{GSH}$ and $N$-acetylcysteine reduced the radical at rates of 24.1 and 29.9 nmoles/min, respectively (Figure 5.3.1). Both GSH and $N$-acetylcysteine scavenged $\mathrm{DPPH}^{\bullet}$ as expected, whereas EdAG was ineffective in reducing $\mathrm{DPPH}^{\bullet}$ to reduced DPPH. EdAG scavenged at a statistically significant slower rate $(2.8 \mathrm{nmoles} / \mathrm{min})$, suggesting that EdAG scavenged radicals by a mechanism different from the sulfhydryl agents, GSH and $N$ acetylcysteine. A two-tailed, unpaired Student's t-test was used to calculate significant differences in reduction rates $(* P<0.05$ for reactions containing EdAG versus GSH control were considered statistically significant). The results of the DPPH radical reduction experiment were similar to the weak or absent reactivity with $\mathrm{DPPH}^{\bullet}$ by other $N$-acyl dehydroalanine compounds (12). 


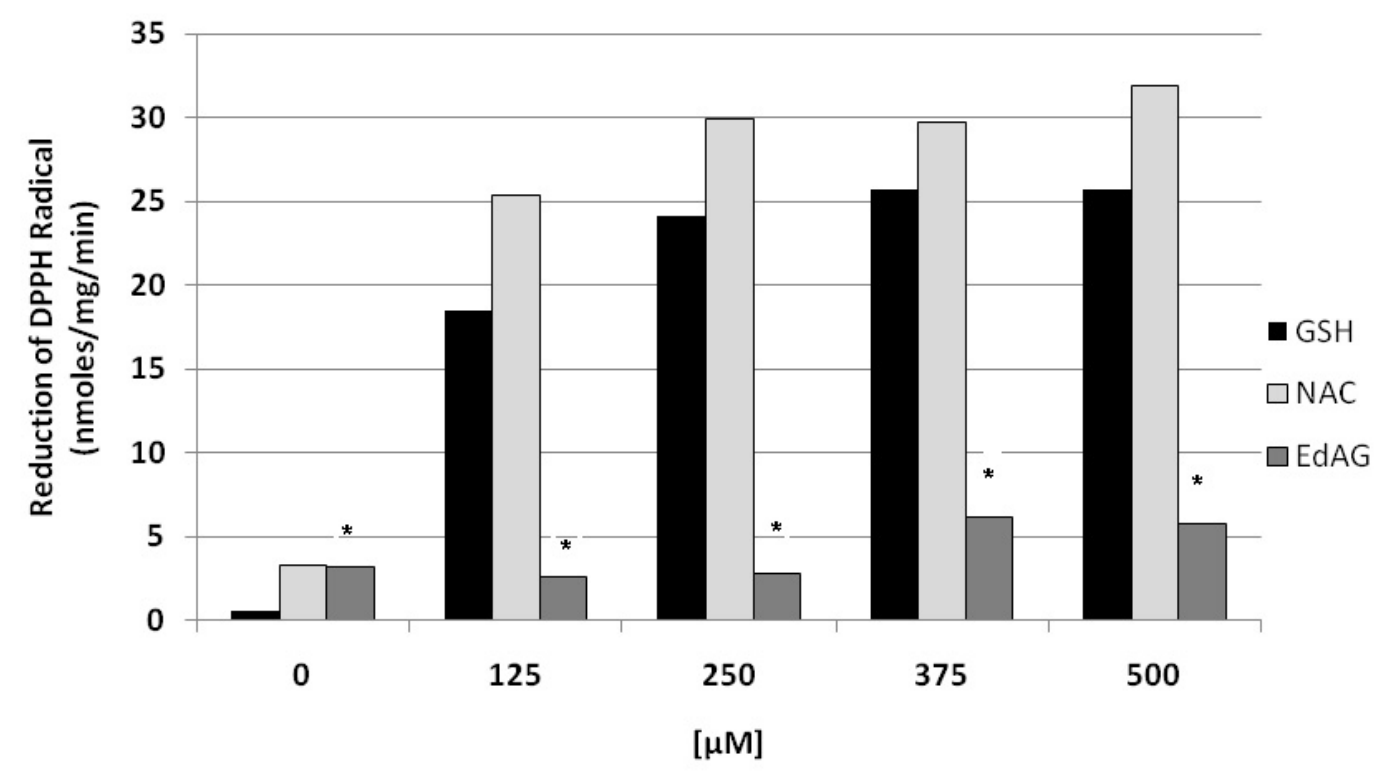

Figure 5.3.1 Reduction of DPPH radical

\subsubsection{Radical scavenging activity of EdAG}

The reactivity of EdAG with hydroxyl radical was determined by generating ${ }^{\bullet} \mathrm{OH}$ with the Fenton reaction in the presence of a spin trap (DMPO or POBN), and EdAG as monitored by EPR. EdAG (0-50 mM) was added to the reaction mixture containing $\mathrm{FeSO}_{4}, \mathrm{DMPO}$, and $\mathrm{H}_{2} \mathrm{O}_{2}$. An obvious decrease in the spin adduct (DMPO-OH $\left.{ }^{\bullet}\right)$ EPR signal was observed in an EdAG concentration-dependent manner, suggesting that EdAG is competing with DMPO for scavenging hydroxyl radical (Figure 5.3.2). A reaction rate constant $\left(\mathrm{k}_{\mathrm{E}}\right)$ of EdAG hydroxyl radical scavenging, determined from the signal height ratio of the signals marked with arrows (Figure 5.3.2 A/B), was calculated to be $8.41 \times 10^{9} \mathrm{M}^{-1} \mathrm{~s}^{-1}$, based on equations in the literature $(14,17)$. Figure 5.3.2 shows typical EPR spectra of the Fenton reaction containing $100 \mathrm{mM}$ DMPO with $0 \mathrm{mM}$ EdAG (Fig. 5.3.2 A), and $100 \mathrm{mM}$ DMPO with $20 \mathrm{mM}$ EdAG (Fig.5.3.2 B). 

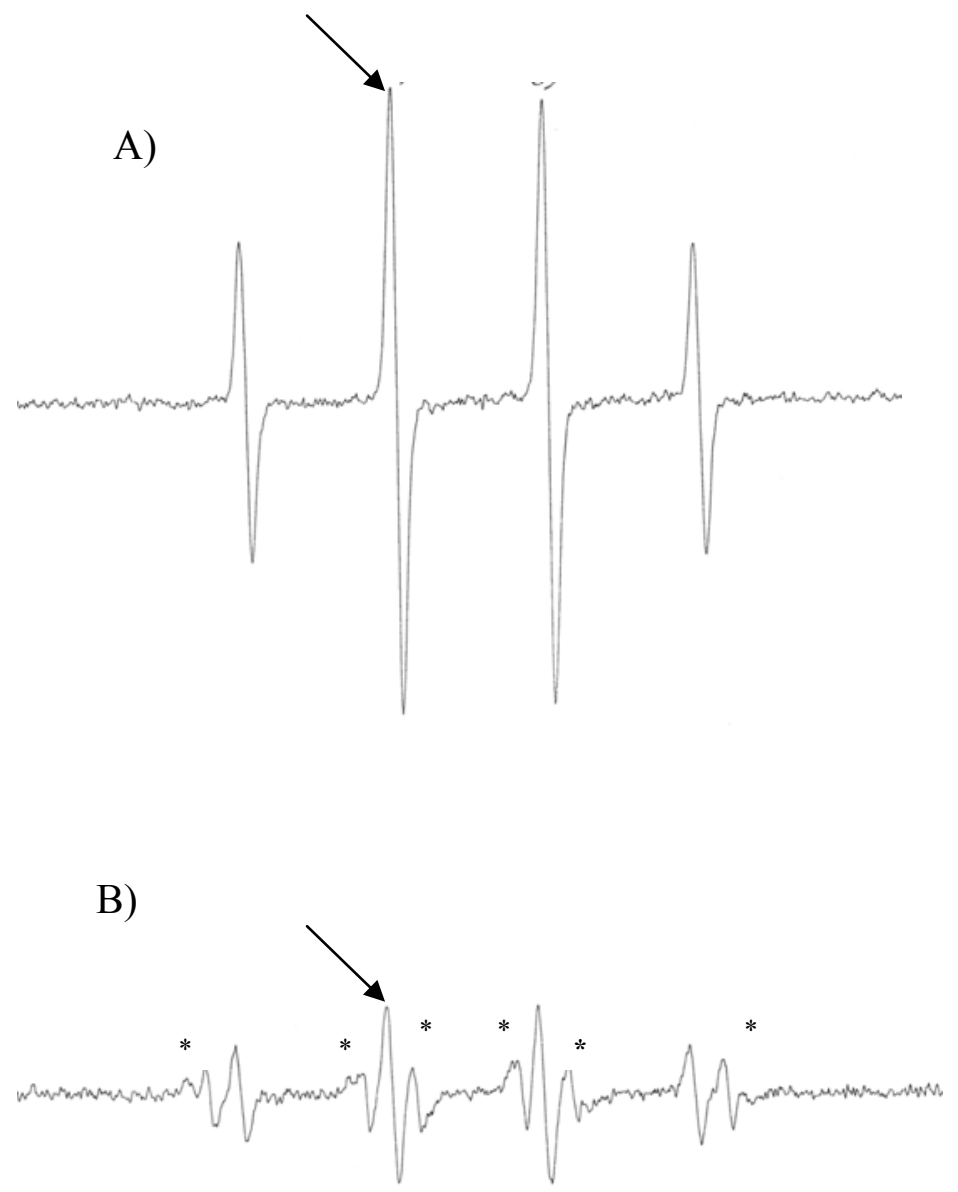

C)

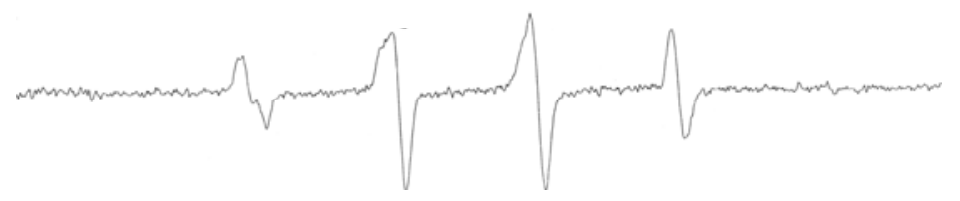

Figure 5.3.2 EPR Spectrum of Fenton Reaction with EdAG 


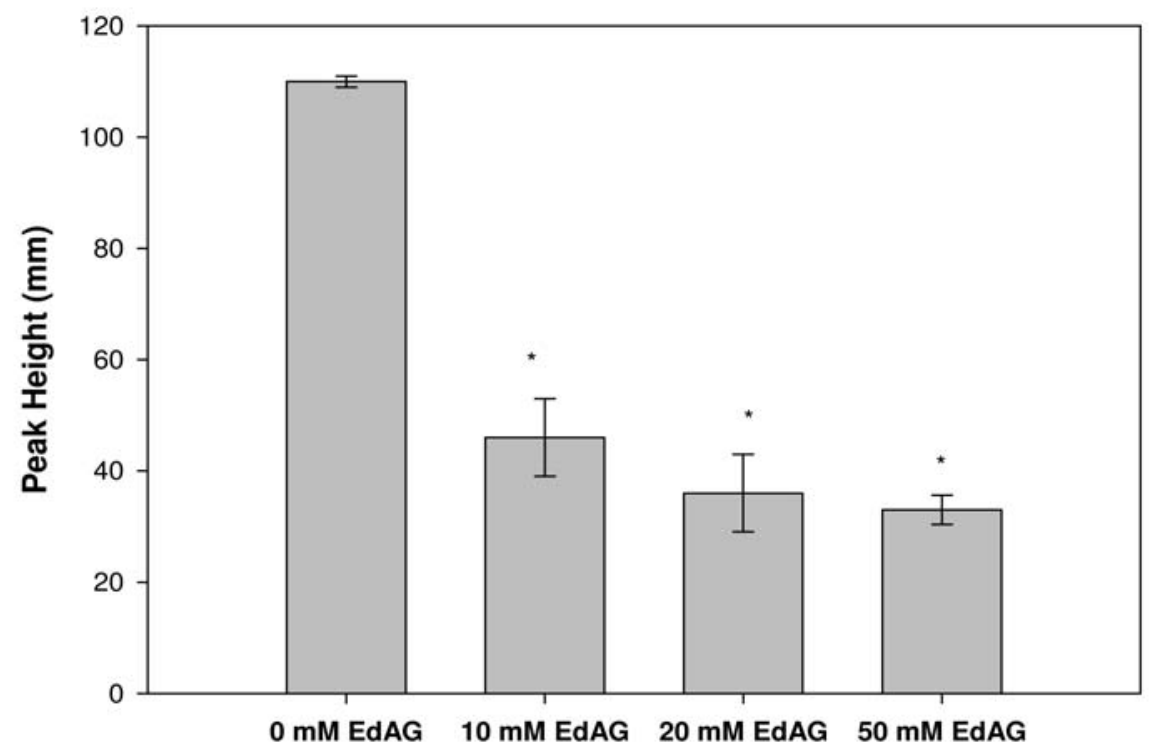

Figure 5.3.3 EdAG-dependent scavenging by EPR

Figure 5.3.3 demonstrates the concentration-dependent reduction in the DMPO-OH${ }^{\bullet}$ signal by EdAG. The signal heights in Figure 5.3.3 are expressed as means \pm SEM with $n=3$ for each group, where a two-tailed, unpaired Student's t-test was used to calculate significant difference (* $P$ value $<0.05$ for reactions containing EdAG versus control without EdAG). EdAG was also suggested to scavenge superoxide $\left(\mathrm{O}_{2}^{-\bullet}\right)$ produced by the oxidation of xanthine by xanthine oxidase. A complete disappearance of the DMPO-O ${ }_{2}^{\bullet}$ signal occurred when $20 \mathrm{mM}$ EdAG was present (data not shown).

Typically, when a radical scavenger is introduced to the Fenton reaction containing DMPO, a simple decrease in the DMPO-OH ${ }^{\bullet}$ EPR signal is observed. When EdAG was the scavenger, the presence of other radical species was indicated by additional absorption bands labeled with asterisks (Fig. 5.3.2B). In comparison, the same reaction containing GSH instead of EdAG produced no signals other than attenuated DMPO-OH signals (Fig. 5.3.2C). EPR 
simulations were performed using WinEPR simulation software and were based on the EPR spectra containing EdAG to aid in the identification of the other radical species present. Analysis of hyperfine splitting patterns and simulated EPR spectra were consistent with three radical species, consisting of $\mathrm{DMPO}-\mathrm{OH}^{\bullet}$ and two carbon-centered radical species. The two carbon-centered radical species are proposed to be diastereomers of DMPO-EdAG-OH${ }^{\bullet}$ (compound III in Scheme 5.3.1) based on a comparison to literature spectra of the products of $N$ acyl dehydroalanines with hydroxyl radical (17) and EPR simulations performed. Scheme 5.3.1 depicts the proposed mechanism of EdAG reaction with hydroxyl radical (I) followed by dimerization (II) or subsequent reaction with DMPO (III).

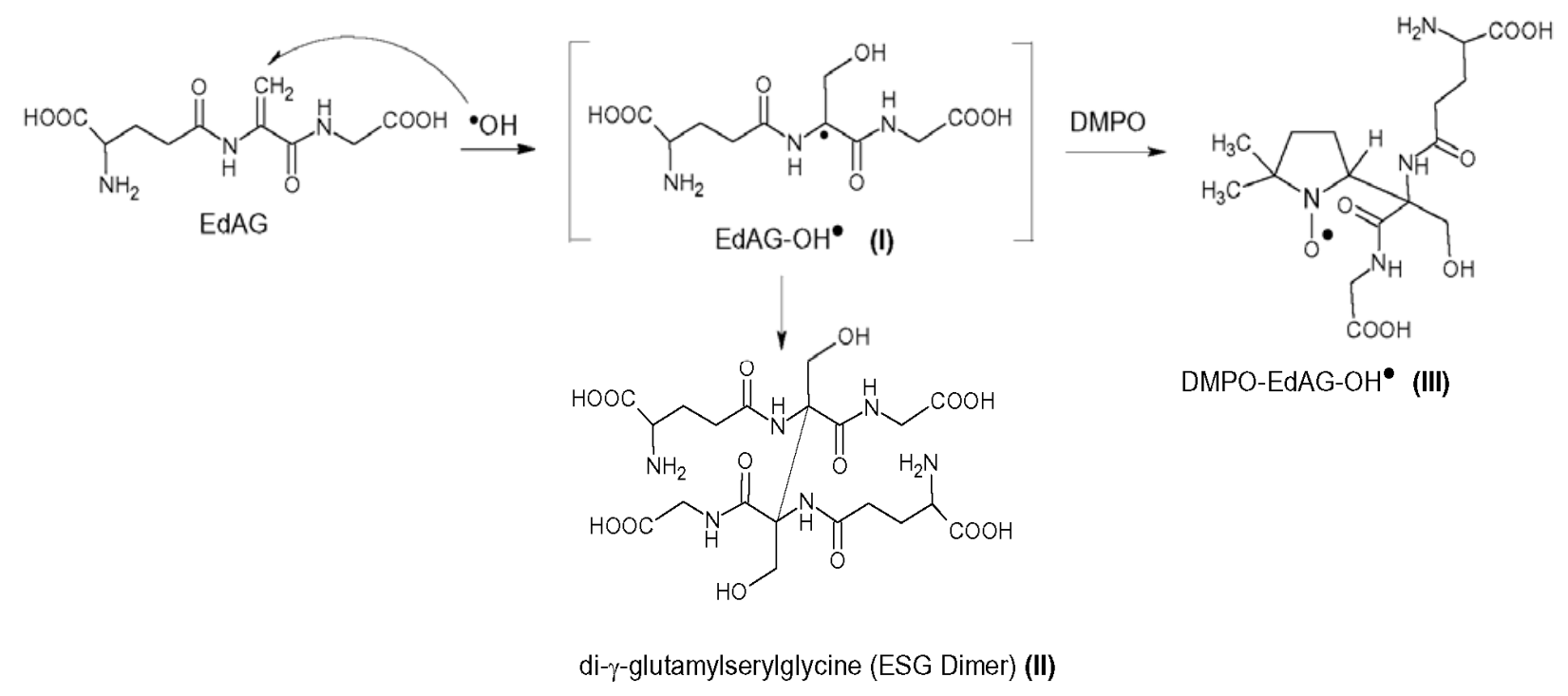

Scheme 5.3.1 Proposed Mechanism of Hydroxyl Radical Scavenging by EdAG 
Figure 5.3.4 depicts the mechanism of hydroxyl radical trapping by the spin trapping agents DMPO (top) and POBN (bottom). POBN was substituted as the spin trap in an attempt to better characterize the carbon-centered radical species. A different EPR spectrum was obtained corresponding to $\mathrm{POBN}-\mathrm{OH}^{\bullet}$ due to the different hyperfine splitting patterns with $\mathrm{POBN}$ compared to DMPO (Fig. 5.3.5A). When EdAG was introduced to the Fenton reaction containing POBN, an obvious decrease in the $\mathrm{POBN}-\mathrm{OH}^{\bullet}$ signal was observed, as well as the presence of another radical species indicated by asterisks (Fig. 5.3.5B). EPR simulations again were performed and it was concluded that the other radical species was a carbon-centered radical that corresponds with the diastereomeric carbon-centered radicals (III) present in the DMPOcontaining reactions, albeit with POBN substituted for DMPO.

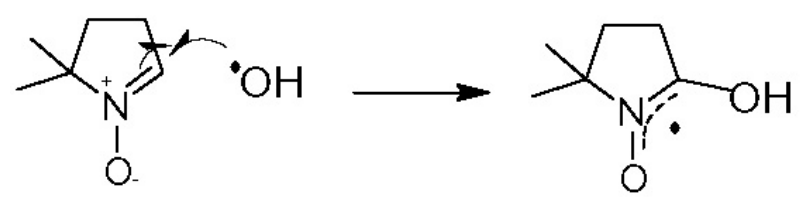<smiles>CC(C)(C)N([O-])C(C)(C)C(C)(C)CCO</smiles>

Figure 5.3.4 Mechanism of hydroxyl radical trapping 
A)

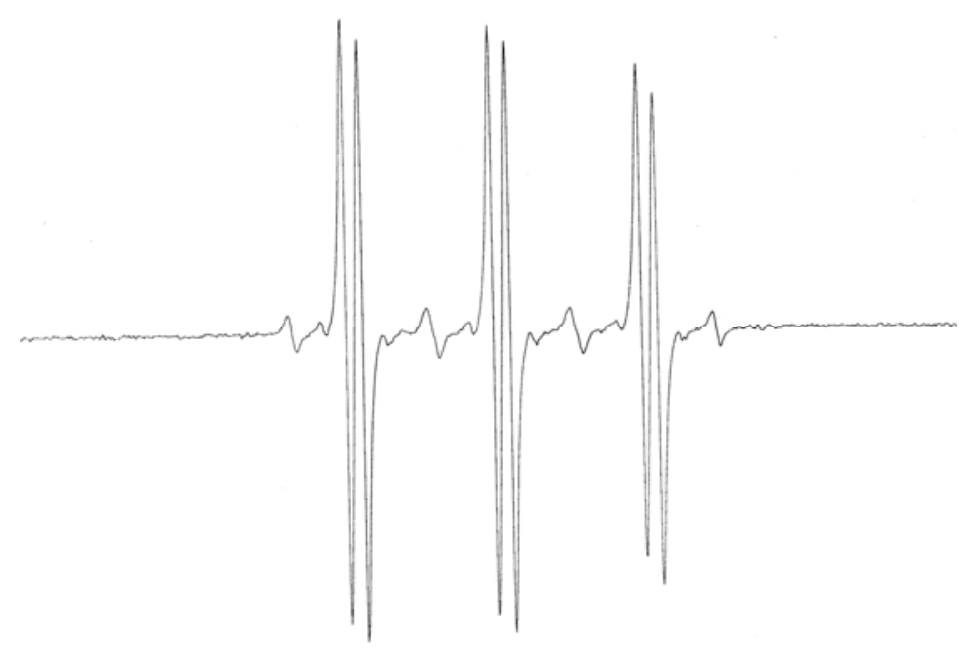

B)

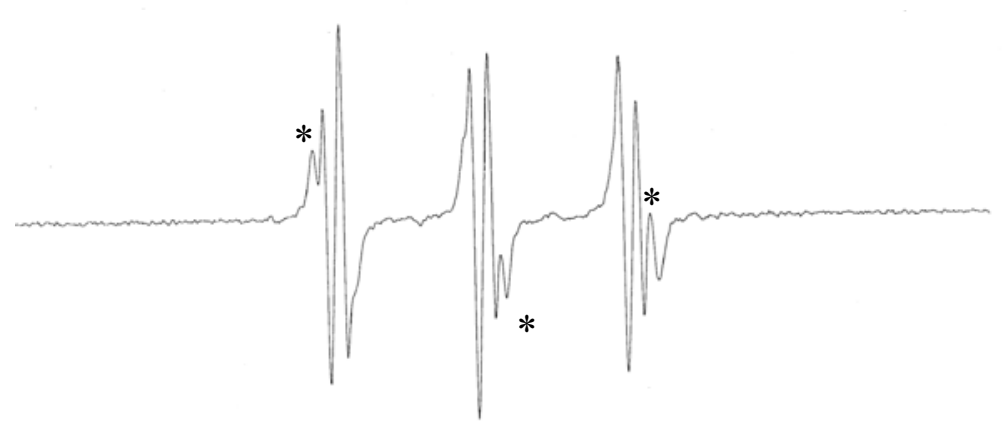

Figure 5.3.5 EPR Spectra of Fenton Reaction and POBN with EdAG

\subsubsection{Mass spectrometric identification of EdAG-hydroxyl radical adduct}

Mass spectrometry was performed to aid in the identification of the adduct of EdAG and • OH. A Fenton reaction mixture containing both $\mathrm{FeSO}_{4}$ and $\mathrm{H}_{2} \mathrm{O}_{2}$ was mixed with EdAG and immediately analyzed by LC/MS to provide structural evidence of the hydroxyl radical conjugated to EdAG (EdAG-OH${ }^{\bullet}$ or $\left.\mathrm{ESG}^{\bullet} ; \mathbf{I}\right)$. Tandem mass spectrometry (MS/MS) was then 
performed on the major reaction product detected in the ion trap mass spectrometer at $\mathrm{m} / \mathrm{z} 581$. The molecular weight of the product ion at $\mathrm{m} / \mathrm{z} 581$ was consistent with a dimer of hydroxylated EdAG (HO-EdAG-EdAG-OH, aka di- $\gamma$-glutamylserylglycine; aka ESG dimer; II) and is in accordance with the literature that carbon-centered radicals formed via captodative mechanisms can dimerize (6).

Assignment of the mass spectral fragmentation ions consistent with the loss of water $(\mathrm{m} / \mathrm{z}$ 563), two water molecules ( $\mathrm{m} / \mathrm{z} 545)$, and pyroglutamic acid plus water ( $\mathrm{m} / \mathrm{z} 434)$ suggest that the dehydroalanine group has reacted with hydroxyl radical forming a serine residue carboncentered radical (ESG) that dimerizes into a di- $\gamma$-glutamylserylglycine compound (Figure 5.3.6). 


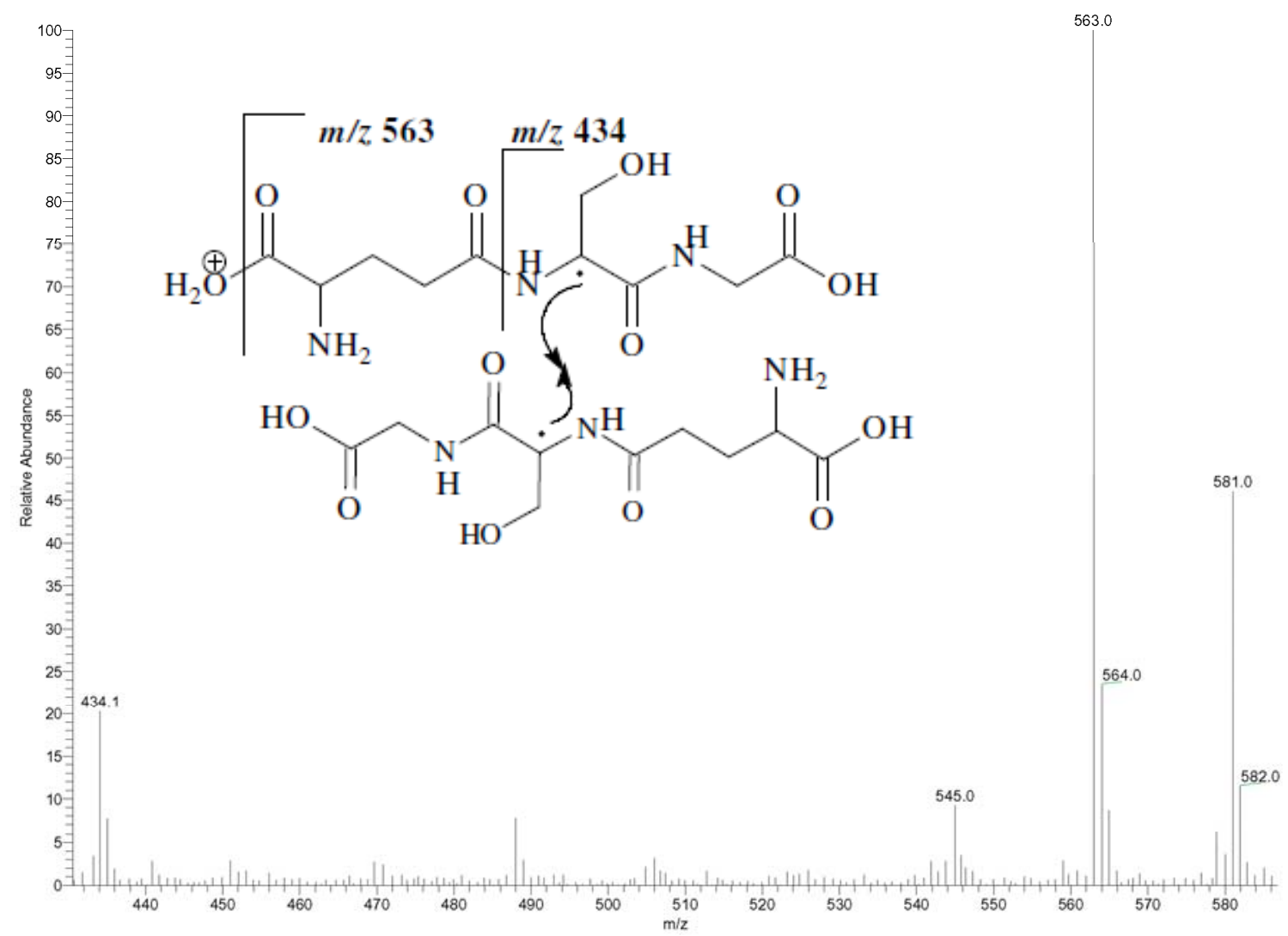

Figure 5.3.6 Accurate MS/MS spectrum of ESG dimer

Scheme 5.3.1 depicts the captodative reaction proposed to occur when EdAG scavenges hydroxyl radical forming the serine analog of glutathione radical intermediate (I) that either dimerizes (II) or is trapped with DMPO (III). The mass spectral evidence supports a mechanism where $\mathrm{ESG}^{\bullet}(\mathbf{I})$ condenses with another I to form a stable ESG dimer (II).

Accurate mass spectrometry, performed on a Fourier-transfer mass spectrometer (FTMS), calculated the theoretical accurate mass for the chemical formula of the ESG dimer (II) as 581.20493 amu. The observed accurate mass spectrum of the dimer from addition of EdAG to the Fenton reaction was $581.20474 \mathrm{amu}$, with a difference of $0.3 \mathrm{ppm}$. FT-MS/MS was 
performed in the accurate mass spectrometer and the resulting product ions were $563.19437 \mathrm{amu}$, 545.18389 amu, and 434.15202 amu, which are consistent with the neutral losses of one water molecule (18.0106 amu), two water molecules (36.0212 amu), and pyroglutamic acid plus water (147.053 amu), respectively. Thus, the major product formed in the reaction of iron, hydrogen peroxide, and EdAG was structurally identified as compound II (Scheme 5.3.1).

\subsubsection{Reaction of EdAG with Hydroxyl Radical ( $\left.{ }^{\circ} \mathrm{OH}\right)$ in RAW 264.7 Cells}

Due to the successful captodative scavenging of ${ }^{\bullet} \mathrm{OH}$ by EdAG in vitro, it was desired to apply this in cells. RAW 264.7 cells were grown overnight in fetal bovine serum (FBS), followed by exposure to EdAG for one hour before addition of chromium to induce ${ }^{\bullet} \mathrm{OH}$ production intracellularly and DMPO to scavenge ${ }^{\bullet} \mathrm{OH}$ radicals and obtain a control EPR signal. EdAG was found to be unable to scavenge hydroxyl radical intracellularly. There was no significant decrease in the DMPO-OH${ }^{\bullet}$ spin adduct signal in the EPR. It was hypothesized that EdAG might be getting conjugated by GSH upon entering the cell, thus forming GSG and eliminating its captodative ability to scavenge hydroxyl radical. A more plausible explanation was that the hydrophilic EdAG was unable to cross the cell membrane into the cell, thus unable to scavenge hydroxyl radicals intracellularly.

\subsubsection{EdAG Cell Permeability}

To determine whether EdAG was traversing the cellular plasma membrane, 6 wells of 500,000 BEAS-2B (bronchial epithelial cells) were plated overnight, then incubated with 100, 200, or $500 \mu \mathrm{M}$ EdAG in either PBS or serum free media (SFM) for 1 hour. After 1 hour, the media was removed and analyzed by LC/MS to determine how much EdAG remained, i.e. how 
much EdAG did not enter cells. Cells were lysed with $0.5 \%$ digitonin for $30 \mathrm{~min}$, centrifuged and then analyzed by LC/MS to determine the amount of EdAG in the cytosol. GSH was detected in the aliquots obtained after digitonin, suggesting that the cells were sufficiently lysed to release intracellular GSH. It was determined that no EdAG was present in the cytosol fraction, indicating that EdAG did not traverse the plasma membrane. Figure 5.3.7 shows that EdAG is present at each concentration level $(100,200$, and $500 \mu \mathrm{M})$, when PBS media supernatent was run on LC/MS prior cell lysing by digitonin (PBS-sn). After the $30 \mathrm{~min}$ exposure to digitonin that lysed cells, the lysate (lys $+\mathrm{d}$, Figue 5.3.6) was run and LC/MS detected no EdAG at any concentration level. The bars corresponding to intracellular EdAG after lysis are not visible because there was no detectable EdAG. This provides an explanation as to why EdAG did not scavenge intracellular hydroxyl radicals as detected by EPR.

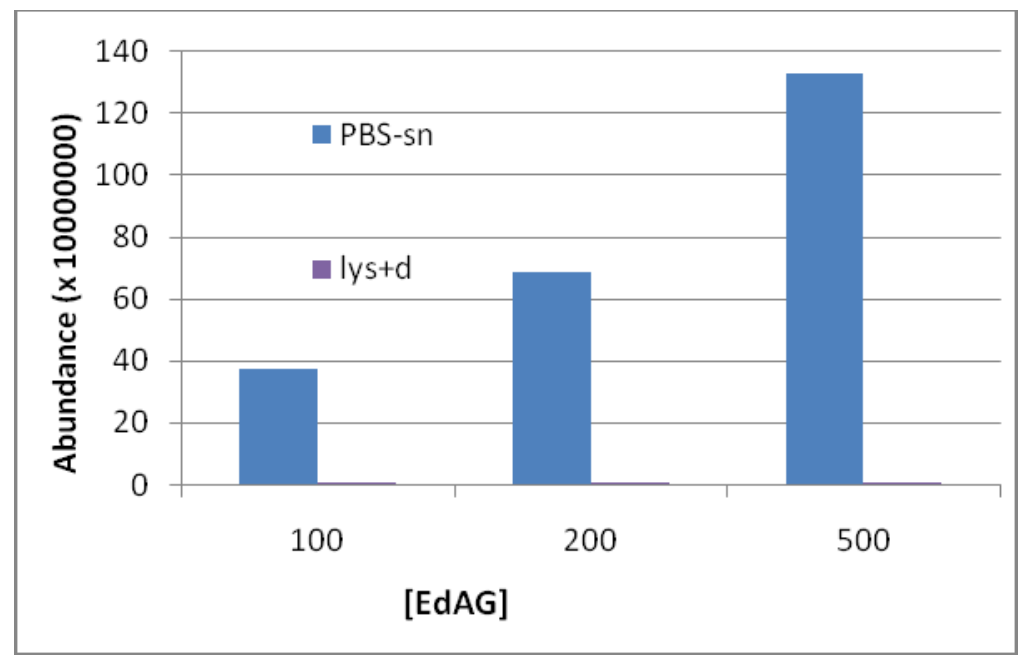

Figure 5.3.7 Cell membrane excludes EdAG 


\section{DISCUSSION}

\section{4}

GSH is an abundant cellular antioxidant that scavenges intracellular ROS (18). In the depleted state of GSH during busulfan treatments, other ROS scavengers may play an increased role. $N$-Acyl dehydroalanine residues have been evaluated for the ability to scavenge hydroxyl radical $(1,12)$ due to the reactivity of the electrophilic dehydroalanine moieties. In addition to EdAG, there are numerous examples of low molecular weight compounds containing dehydroalanine moieties that are able to scavenge ROS such as hydroxyl $\left({ }^{\bullet} \mathrm{OH}\right)$ and superoxide $\left(\mathrm{O}_{2}^{-\bullet}\right)$ radical, as well as reducing lipid peroxidation $(1,4,7-12,17,19)$.

As is the case for $\mathrm{N}$-acyl dehydroalanine esters, compounds operating though a captodative mechanism would not be expected to reduce DPPH radical due to the lack of a redox-active thiol group present in GSH. Consistent with other dehydroalanines (12), EdAG did not scavenge $\mathrm{DPPH}^{\bullet}$, which suggests that EdAG is not a redox-based scavenger. Thus, EPR and mass spectrometry (MS) were used to determine the mechanism by which EdAG scavenges radicals.

EdAG scavenged ${ }^{\bullet} \mathrm{OH}$ generated in the Fenton reaction as shown by attenuation of the EPR signal from spin-trapped radicals with DMPO (Figure 5.3.2). EdAG also significantly attenuated the DMPO- $\mathrm{O}_{2}{ }^{\bullet}$ signal (data not shown), suggesting that EdAG also scavenged superoxide as has been observed for other dehydroalanine scavengers of ROS (1). The radical intermediate formed, EdAG-OH${ }^{\bullet}(\gamma$-glutamylserylglycine; I), stabilizes the free radical on the $\alpha$ carbon of the resulting serine residue, where it is too unreactive to attack the alkene of another EdAG, and in a recombination reaction, dimerizes to form compound II. The rate of the concentration-dependent scavenging of ${ }^{\bullet} \mathrm{OH}$ by EdAG $\left(\mathrm{k}_{\mathrm{E}}\right)$ was calculated as $8.41 \times 10^{9} \mathrm{M}^{-1} \mathrm{~s}^{-1}$. 
This rate is comparable to the scavenging rate constant of the $N$-acyl dehydroalanine AD- 5 of 4.5 x $10^{9} \mathrm{M}^{-1} \mathrm{~s}^{-1}$ (17) and of the redox active resveratrol $9.45 \times 10^{8} \mathrm{M}^{-1} \mathrm{~s}^{-1}$ (14). The presence of additional radical species in Figure 5.3.2B was consistent with literature (17) and EPR simulations were performed to better characterize those radical species. Based on the known hyperfine splitting patterns for $\mathrm{DMPO}-\mathrm{OH}^{\bullet}\left(a_{\mathrm{N}} \cong a_{\mathrm{H}} \cong 14.9\right.$ gauss $)$, there were two additional radical species suggested to be present with hyperfine splittings consistent with $a_{\mathrm{N}}>a_{\mathrm{H}}$, which indicated carbon-centered radicals. Further analysis of the simulation results revealed that the two carbon-centered radicals present resembled diastereomers of the same compound, suggested to be compound III from Scheme 5.3.1.

A second spin trap, POBN, was used to further characterize the hydroxyl radical scavenging properties of EdAG and assess the validity of diastereomers of spin traps adducted to compound $\mathbf{I}$. The use of a different spin trap aside from DMPO could help differentiate each carbon-centered radical species by EPR. Figure 5.3.5B does show the presence of another radical species (other than $\mathrm{POBN}-\mathrm{OH}^{\bullet}$ ) as indicated by asterisks, but EPR simulations determined there to be only one carbon-centered radical species present, not two as the case when DMPO was the spin trap. It was concluded that both spin traps adduct compound $\mathbf{I}$ at a chiral center, however POBN produces only one carbon-centered radical species detectable by EPR whereas DMPO produces diastereomers of the same carbon-centered radical species. Alternatively, POBN could be producing diastereomers that have signals that are superimposed. Mass spectrometric identification of the dimer (II) as a product in the Fenton reaction with EdAG supports the indication that a captodative radical intermediate is likely to be formed in the reaction of EdAG with hydroxyl radical (Scheme 5.3.1). Spin traps (DMPO, POBN) were excluded from these experiments so to not produce spin trapped carbon-centered radicals, thus 
leaving only the EdAG-hydroxyl radical product. Confirmation of the structure was performed by FT-MS/MS for accurate mass neutral loss, where II lost fragments of water and pyroglutamic acid. Pyroglutamic acid is a common neutral loss for GSH-containing compounds when analyzed by ESI-MS/MS $(20,21)$. The simplicity of the MS/MS product ion spectrum of II (di$\gamma$-glutamylserylglycine) as shown in Figure 5.3.6 suggests a symmetrical compound.

A mechanism involving an energetically favorable captodative radical is a plausible explanation for the Michael addition of hydroxyl radical to EdAG to form a carbon-centered radical on the $\alpha$-carbon of the dehydroalanine moiety. This captodative, or push-pull radical (5), is stabilized by resonance associated with electron donation by the adjacent nitrogen atom, and delocalization through the electron-withdrawing carbonyl group. This reactive radical (I) can condense with a second radical to form an ESG dimer (II), or can be trapped with DMPO (III). The dimerization is in accordance with previous studies involving hydroxyl radical and dehydroalanine-containing compounds (6). It is interesting to note that the captodative mechanism for scavenging radicals is different from the scavenging ability of sulfur-containing compounds, most notably GSH and $N$-acetylcysteine, which involve thiyl radical intermediates in a redox mechanism.

Because in vitro experiments were successful in determining the ability of EdAG to captodatively trap hydroxyl radical, it was desired to apply this to a physiological setting to determine if EdAG, when formed inside hepatocytes during busulfan metabolism, is able to scavenge hydroxyl radicals intracellularly. During high-dose busulfan administration, GSH can become depleted, leaving the cell vulnerable to ROS. EdAG could potentially play a protective role by scavenging ROS. EdAG was not shown to decrease the hydroxyl radical signal. When further investigated as to why this occurred, it was hypothesized that the hydrophilic EdAG was 
not capable of crossing the cell membrane. EdAG is a GSH analog and there are no cellular GSH importers because each of the three individual amino acids (glutamate, cysteine, glycine) are assembled intracellularly. Following this rationale, the cell permeability of EdAG was tested on a bronchial epithelial cell line, which was chosen because of its accessibility (plated cells were courtesy of George Medan). After a 1 hour incubation of EdAG with plated BEAS cells, there was no intracellular EdAG detected by LC/MS. The hydrophilic nature of EdAG is suggested to prohibit the intact molecule from freely crossing the cell membrane, and thus from scavenging intracellular hydroxyl radical.

In conclusion, it was demonstrated in this chapter that the dehydroalanine-containing EdAG can scavenge hydroxyl radical by a captodative mechanism that is in agreement with literature references for other dehydroalanine-containing compounds. EdAG induced a concentration-dependent scavenging of hydroxyl radical as monitored by EPR. The radical product was structurally identified by mass spectrometry. Physiologically, this is relevant because as busulfan is metabolized by GSH conjugation to form EdAG, numerous mechanisms contribute to the depletion of intracellular glutathione. Glutathione plays an essential role as a cellular redox buffer and its depletion could increase oxidation by ROS. The transformation of GSH to EdAG represents the loss of thiol-related redox scavenging but the addition of a captodative scavenging mechanism. Thus, EdAG is formed intracellularly and hypothetically is present to scavenge intracellular hydroxyl and superoxide radicals in the cytosol. The scope of this research did not extend to inducing ROS production in hepatocytes while metabolizing busulfan to form EdAG. 


\section{REFERENCES}

(1) Buc-Calderon, P., and Roberfroid, M. (1988) Inhibition of O2-.- and HO.- mediated processes by a new class of free radical scavengers: the $\mathrm{N}$-acyl dehydroalanines. Free Radic. Res. Commun. 5(3), 159-168.

(2) Molyneux, P. (2004) The use of the stable free radical diphenylpicrylhydrazyl (DPPH) for estimating antioxidant activity. Songklanakarin J.Sci.Technol. 26, 211-219.

(3) Hopkinson, A. C. (2009) Radical cations of amino acids and peptides: structures and stabilities. Mass Spectrom. Rev. 28(4), 655-671.

(4) Sipe, H. J., Buc-Calderon, P., Roberfroid, M., and Mason, R. P. (1993) Identification of the free radical formed by addition of hydroxyl radical to dehydroalanine compounds. Chem. Biol. Interact. 86(2), 93-102.

(5) Viehe, H. G., Merenyi, R., Stella, L., and Janousek, Z. (1979) Capto-dative substituent effects in synthesis with radicals and radicophiles. Angew. Chem. 18, 917-932.

(6) Roberfroid, M., Viehe, H. G., and Remacle, J. (1987) (Testa, B., Ed.) pp 1-84, Academic Press,

(7) Allemon, A. M., Buc, C. P., and Roberfroid, M. (1987) Potentiation of the therapeutic activity of cyclophosphamide by an original N-acyldehydroalanine. Drugs Exp. Clin. Res. 13(6), 359-365.

(8) Buc-Calderon, P., Praet, M., Ruysschaert, J. M., and Roberfroid, M. (1987) Free radical modulation by N-substituted dehydroalanines, a new way to improve therapeutic activity of anticancer drugs. Cancer Treat. Rev. 14(3-4), 379-382. 
(9) Buc-Calderon, P., and Roberfroid, M. (1989) Inhibition of rat liver microsomal lipid peroxidation by $\mathrm{N}$-acyldehydroalanines: an in vitro comparative study. Arch. Biochem. Biophys. 273(2), 339-346.

(10) Buc-Calderon, P., Defresne, M. P., Barvais, C., and Roberfroid, M. (1989) N-acyl dehydroalanines protect from radiation toxicity and inhibit radiation carcinogenesis in mice. Carcinogenesis 10(9), 1641-1644.

(11) Ghoshal, A. K., Rushmore, T. H., Buc-Calderon, P., Roberfroid, M., and Farber, E. (1990) Prevention by free radical scavenger AD5 of prooxidant effects of choline deficiency. Free Radic. Biol. Med. 8(1), 3-7.

(12) Suzen, S., Gurkok, G., and Coban, T. (2006) Novel N-acyl dehydroalanine derivatives as antioxidants: studies on rat liver lipid peroxidation levels and DPPH free radical scavenging activity. J. Enzyme Inhib. Med. Chem. 21(2), 179-185.

(13) Vo, T. K., Fischer, S. M., and Slaga, T. J. (1991) Effects of N-acyl dehydroalanines on phorbol ester-elicited tumor development and other events in mouse skin. Cancer Lett. 60(1), 25-32.

(14) Leonard, S. S., Xia, C., Jiang, B. H., Stinefelt, B., Klandorf, H., Harris, G. K., and Shi, X. (2003) Resveratrol scavenges reactive oxygen species and effects radical-induced cellular responses. Biochem. Biophys. Res. Commun. 309(4), 1017-1026.

(15) Shi, X., Ye, J., Leonard, S. S., Ding, M., Vallyathan, V., Castranova, V., Rojanasakul, Y., and Dong, Z. (2000) Antioxidant properties of (-)-epicatechin-3-gallate and its inhibition of $\mathrm{Cr}(\mathrm{VI})$-induced DNA damage and $\mathrm{Cr}(\mathrm{IV})$ - or TPA-stimulated NFkappaB activation. Mol. Cell Biochem. 206(1-2), 125-132. 
(16) Mariott, P. R., Perkins, M. J., and Griller, D. R. (1980) Spin trapping of hydroxyl in water: A kinetic evaluation of two popular traps. Canadian Journal of Chemistry 58, 803-807.

(17) Buc-Calderon, P., Sipe, H. J., Jr., Flitter, W., Mason, R. P., and Roberfroid, M. (1990) $\mathrm{N}$-acyl dehydroalanines scavenge oxygen radicals and inhibit in vitro free radical mediated processes. Chem. Biol. Interact. 73(1), 77-88.

(18) Lopez-Mirabal, H. R., and Winther, J. R. (2008) Redox characteristics of the eukaryotic cytosol. Biochim. Biophys. Acta 1783(4), 629-640.

(19) Buc-Calderon, P., and Roberfroid, M. (1990) AD 5, a dehydroalanine derivative, decreases the amount of reactive oxygen species formed during nitrofurantion microsomal metabolism. Life Sci. 46(3), 207-215.

(20) Castro-Perez, J., Plumb, R., Liang, L., and Yang, E. (2005) A high-throughput liquid chromatography/tandem mass spectrometry method for screening glutathione conjugates using exact mass neutral loss acquisition. Rapid Commun. Mass Spectrom. 19(6), 798-804.

(21) Zhu, M., Ma, L., Zhang, H., and Humphreys, W. G. (2007) Detection and structural characterization of glutathione-trapped reactive metabolites using liquid chromatography-high-resolution mass spectrometry and mass defect filtering. Anal. Chem. 79(21), 8333-8341. 


\section{CURRICULUM VITAE}

Cody J. Peer

18300 Feathertree Way, \#303

Montgomery Village, MD 20886

Phone: 412-736-6351

E-mail: cjpeer28@yahoo.com

\section{EDUCATION:}

August 2005 - December 2009

Ph.D. Candidate in Pharmaceutical and Pharmacological Sciences

Department of Basic Pharmaceutical Sciences,

School of Pharmacy, West Virginia University

Research Advisor: Patrick S. Callery, Ph.D.

\section{August 2001-May 2005}

B.S. in Chemistry

Department of Chemistry, Dickinson College

Carlisle, PA 17013

Advisor: Cindy Samet, Ph.D. Associate Professor of Chemistry

\section{RESEARCH FOCUS:}

- The reactivity of a specific metabolite of the anticancer drug busulfan, which is an electrophilic dehydroalanine analog of glutathione. Determining the mechanisms by which this metabolite induces toxicity by studying enzyme inhibition and conjugation with endogenous nucleophiles, free radicals, and the enzyme it inhibits

- Pharmacokinetics of the chemotherapeutic agent 5-fluorouracil and its oral prodrug tegafur in Phase I clinical trials using validated high-performance liquid chromatography/tandem mass spectrometry (LC/MS/MS)

\section{RESEARCH SKILLS:}

- Extensive experience with HPLC, MS, LC/MS, and UV spectrophotometry

- Method validation for LC/MS/MS

- Pharmacokinetic analysis of patient plasma samples with a validated LC/MS/MS method

- Hands on experience with matrix-assisted laser desorption ionization (MALDI) mass spectrometry, electron paramagnetic resonance (EPR) and nuclear magnetic resonance (NMR) spectroscopy

\section{CERTIFICATIONS/LABORATORY TRAINING:}

- HIPAA, West Virginia University, Morgantown WV 26506

- WVU Animal Welfare Training according to the IACUC, October 2005, West Virginia University, Morgantown, WV 26506

- Scientific Ethics Training (Fall 2005)

\section{AWARDS/SCHOLARSHIPS:}

- E.J. Van Liere Memorial Research Convocation and Research Day (WVU Health Sciences) $\$ 100$ award for oral presentation in April 2009 
- WVU School of Pharmacy, Pharmaceutical and Pharmacological Sciences Graduate Student Research award - \$250 awarded in April 2009

- Robert E. Stitzel Graduate Student Support Fund (WVU School of Pharmacy) - Research and Scholarship Productivity Award: \$1,000 award granted in September 2008

- Society of Forensic Toxicologists (SOFT) Educational Research Award: \$1,000 travel award granted in May 2007 for the October 2007 Annual National Meeting in Raleigh-Durham, NC

- Pennsylvania Higher Education Assistance Agency (PHEAA), New Economy Technology Scholarship (NETS), SciTech Scholarship: \$3,000 grant awarded in 2002, 2003 and 2004

\title{
PROFESSOINAL EXPERIENCE:
}

\section{June - August 2005 and May - August 2004}

Mylan Pharmaceuticals, Morgantown, WV. Sponsor: Scott Chervenick, Ph.D.

Prepared clinical plasma samples for bioanalytical analysis by HPLC and MS, as well as reagents, solutions, and mobile phases used in HPLC and MS.

\author{
August - December 2004 \\ Department of Chemistry, Dickinson College, Carlisle, PA. Lab of Michael Holden, Ph.D. \\ Synthesized ferrocene-containing analogs, analyzed by NMR, to study iron analogs of the anti-malarial \\ drug proguanil.

\section{June - August 2003} \\ Center for Pharmaceutical Technology, Mylan School of Pharmacy, Duquesne University, Pittsburgh, \\ PA. Sponsor: Carl Anderson, Ph.D. \\ Assisted graduate students in projects consisting of using Near-Infrared (NIR) imaging to determine the \\ composition of pharmaceutical compacts.
}

\section{PROFESSIONAL MEMBERSHIPS:}

- International Society for the Study of Xenobiotics (ISSX) (June 2008-Present)

- Society of Forensic Toxicologists (SOFT) (May 2007-Present)

- American Society for Mass Spectrometry (ASMS) (November 2006-Present)

- Gamma Sigma Epsilon Chemistry Honor Society, Rho Zeta Chapter, Dickinson College, Carlisle, PA 17013 (May 2004)

\section{PUBLICATIONS:}

Leonard SS, Hogans VJ, Coppes-Petricorena Z, Peer CJ, Vining TA, Fleming DW, and Harris GK. Analysis of Free Radical Scavenging of Yerba Mate (Ilex paraguriensis) using Electron Spin Resonance and Radical-induced DNA Damage. J Food Science. 00: C1-C7 (2009).

Miranda L. Hanson, Cody J. Peer, Rodney Brundage, Patrick Callery, Kathleen Brundage, Rosana Schafer, Sergei Eremin, and John B. Barnett. Subcellular localization of the amide class herbicide 3,4dichloropropionanilide (DCPA) in T cells and hepatocytes. J. Tox. Envir. Htl. Part A: 73(1):1-4 (2009).

Erinne R. Dabkowski, Courtney L. Williamson, Valerie C. Bukowski, Rebecca S. Chapman, Stephen S. Leonard, Cody J. Peer, Patrick S. Callery, and John M. Hollander. Diabetic Cardiomyopathy-Associated Dysfunction in Spatially Distinct Mitochondrial Subpopulations Am J Physiol Heart Circ Physiol. 296(2):H359-69 (2009).

Islam R. Younis, Meenal Elliott, Cody J. Peer, Arthur J.L. Cooper, John T. Pinto, Gregory 
W. Konat, Michal Kraszpulski, William P. Petros, and Patrick S. Callery. Dehydroalanine analog of glutathione: An electrophilic busulfan metabolite that binds to human glutathione S-transferase A1-1. J. Pharmacol Exp Ther. 327(3): 770-6 (2008).

Cody J. Peer, David J. Clay, Heather L. Glover, Kristina L. Renninger, James C. Kraner, and Patrick S. Callery. Direct Injection Mass Spectrometric Confirmation of Multiple Drugs in Overdose Cases from Postmortem Blood using ESI-MS/MS and MS ${ }^{3}$. J. Anal. Toxicol. 32(8): 709-714 (2008).

Cody J. Peer, Diaa M. Shakleya, Islam R. Younis, James C. Kraner, and Patrick S. Callery. DirectInjection Mass Spectrometric Method for the Rapid Identification of Fentanyl and Norfentanyl in Postmortem Urine of Six Drug-Overdose Cases. J. Anal. Toxicol. 31(8): 515-520 (2007).

\section{ABSTRACTS:}

Cody J. Peer, Islam R. Younis, Valerie C. Bukowski, Stephen S. Leonard, William P. Petros, and Patrick S. Callery. Gamma-Glutamyldehydroalanylglycine is an Unusual Metabolite of Glutathione that Inhibits Glutathione S-Transferase Activity and Reacts with Hydroxyl Radical by a Captodative Mechanism.

Accepted for poster presentation at the $16^{\text {th }}$ Annual North American Regional Meeting of the International Society for the Study of Xenobiotics (ISSX), October 19-21, 2009, Baltimore, MD.

Cody J. Peer, Steven S. Leonard, and Patrick S. Callery. Properties of a Dehydroalanine Analog of Glutathione: A Novel Busulfan Metabolite. Presented as an oral and poster presentation at the 2009 West Virginia University Health Sciences Van Liere Research Day. April 27-28, 2009.

William Petros, Mukur Gupta, Vincenzo Guarcello, Thomas McIntire, Terrance McManus, Cody Peer, Gerry Hobbs, Scot Remick, William Peters. Preclinical development of a topical eniluracil formulation for prevention of fluropyrimidine-induced hand foot syndrome (abstract). American Association for Cancer Research, Dec. 2, 2008.

Cody J. Peer, Islam R. Younis, and Patrick S. Callery. Formation of a Non-Reducible Lanthionine Thioether from Glutathione and a Busulfan Metabolite (Poster). Presented as a poster at the $15^{\text {th }}$ Annual North American Regional Meeting of the International Society for the Study of Xenobiotics (ISSX), October 12-16, 2008, San Diego, CA.

Islam R. Younis, Cody J. Peer, Meenal Elliot, Arthur J. L. Cooper, John T. Pinto, Gregory W. Konat, William P. Petros, and Patrick S. Callery. Dehydroalanine Analog of Glutathione: An Electrophilic Busulfan Metabolite that Binds to Human Glutathione-S-Transferase A1-1. Presented in an oral format at the $41^{\text {st }}$ Annual Mid-Atlantic Graduate Student Symposium in Medicinal Chemistry (MAGSS), July 2022, 2008, Wayne State University, Detroit, MI.

Miranda L. Hanson, Cody J. Peer, Kathleen Brundage, Rosana Schafer, Sergei A. Eremin, and John B. Barnett. Subcellular Localization of the Amide Class Herbicide 3,4-Dichloropropionanilide (DCPA) in T Cells. Presented as a poster at the $47^{\text {th }}$ Annual Meeting of the Society of Toxicology, March 16-20, 2008, Seattle, WA.

Cody J. Peer, Diaa M. Shakleya, Islam R. Younis, James C. Kraner, and Patrick S. Callery. DirectInjection Mass Spectrometric Method for the Rapid Identification of Fentanyl and Norfentanyl in Postmortem Urine of Six Drug-Overdose Cases. Presented as a poster at the Society of Forensic Toxicologists (SOFT) Annual Meeting, October 14-19, 2007, Chapel Hill, NC. 
Jirapan Moungjaroen, Islam R. Younis, Cody J. Peer, Ubonthip Nimmannit, Yon Rojanasakul, and Patrick S. Callery. Mechanism of Lipoic Acid Protection from Cisplatin-induced DNA Damage Using HPLC and Mass Spectrometry. Presented as a poster at the $40^{\text {th }}$ Annual Mid-Atlantic Graduate Student Symposium in Medicinal Chemistry (MAGSS), June 10-12, 2007, West Virginia University, Morgantown, WV.

Cody J. Peer, Diaa M. Shakleya, Islam R. Younis, James C. Kraner, and Patrick S. Callery. DirectInjection Mass Spectrometric Method for the Rapid Identification of Fentanyl and Norfentanyl in Postmortem Urine of Six Drug-Overdose Cases. Presented as a poster at the $55^{\text {th }}$ Annual Meeting of the American Society for Mass Spectrometry (ASMS), June 3-7, 2007, Indianapolis, IN.

Cody J. Peer, Patrick S. Callery, James C. Kraner, and Diaa M. Shakleya. Identification of 8 Drugs in 15 Minutes from Postmortem Urine by Direct Infusion MS/MS. Case Note published in May 2007 Issue of ToxTalk, part of the Society of Forensic Toxicologists.

Diaa M. Shakleya, James C. Kraner, Cody J. Peer, and Patrick S. Callery. Identification of Fentanyl in Urine from Drug Abuse Cases Using a Direct Multistage Mass Spectrometry Method. Presented as a poster at the $39^{\text {th }}$ Annual Mid-Atlantic Graduate Student Symposium in Medicinal Chemistry (MAGSS), June 18-20, 2006, Ohio State University, Columbus, OH.

Carl Anderson, David Molseed, Cody J. Peer. Quantitative Imaging of Pharmaceutical Compacts. Duquesne University, Center for Pharmaceutical Technology (DCPT), Pittsburgh, PA. Presented at the 2004 IFPAC/PAT meeting on January 14, 2004.

\section{REFERENCES:}

Patrick S. Callery, Ph.D. Professor and Dissertation Advisor, Department of Basic Pharmaceutical Sciences and Assistant Dean for Research and Graduate Programs, School of Pharmacy, West Virginia University. P.O. Box 9530, Morgantown, WV 26506. Phone: (304) 293-1482. Fax: (304) 293-2576. Email: pcallery@hsc.wvu.edu

William P. Petros, Pharm.D., FCCP. Mylan Professor of Pharmacology, Associate Director for AntiCancer Drug Development, Mary Babb Randolph Cancer Center, Department of Basic Pharmaceutical Sciences, School of Pharmacy, West Virginia University. P.O. Box 9530, Morgantown, WV 26506. Phone: (304) 293-0495. Fax: (304) 293-4667. Email: wpetros@hsc.wvu.edu

Peter M. Gannett, Ph.D. Professor of Medicinal Chemistry, Acting Chair of the Department of Basic Pharmaceutical Sciences, School of Pharmacy, West Virginia University. P.O. Box 9530, Morgantown, WV 26506. Phone: (304) 293-1480. Fax: (304) 293-2576. Email: pgannett@hsc.wvu.edu

Stephen S. Leonard, Ph.D. Research biologist at the National Institute for Occupational Safety and Health (NIOSH), 1095 Willowdale Rd, M/S 2015, Morgantown, WV 26505. Phone: (304) 285-5831. Fax: (304) 285-5938. Email: sel5@cdc.gov

Fred L. Minnear, Ph.D. Assistant Dean for Graduate Studies, Director of MD/Ph.D Scholars Program for the School of Medicine, Professor of Physiology and Pharmacology, School of Medicine, West Virginia University. P.O. Box 9229, Morgantown, WV 26506. Phone: (304) 293-6229. Fax: (304) 2937038. Email: $\underline{\text { minnear@hsc.wvu.edu }}$ 
Justin Hettick, Ph.D. Research chemist at the National Institute for Occupational Safety and Health (NIOSH), 1095 Willowdale Rd, MS L-4020, Morgantown, WV 26505. Phone: (304) 285-6065. Fax: (304) 285-6126. Email: jhettick@cdc.gov

Islam R. Younis, Ph.D. Clinical Pharmacologist, Division of Clinical Pharmacology I, Office of Clinical Pharmacology, OTS, CDER, US Food and Drug Administration, 10903 New Hampshire Avenue, Silver Spring, MD 20993. Phone: (301)796-3657. Fax: (301)796-9992. Email: islam.younis@,fda.hhs.gov 